DOE/ER- -0529F

DE92 004708

\title{
Summaries of FY 1991 Engineering Research
}

November 1991

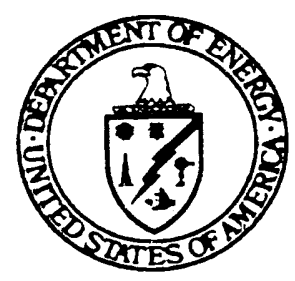

U.S. Department of Energy

Office of Energy Research Office of Basic Energy Sciences Division of Engineering and Geosciences Washington, D. C. 20585 


\section{Foreword}

This report documents the BES Engineering Research Program for fiscal year 1991; it provides a summary for each of the program projects in addition to a brief program overview. The report is intended to provide staff of Congressional committees, other executive departments. and other DOE offices with substantive program information so as to facilitate governmental overview and coordination of Federal research programs. Of equal importance, its availability facilitates communication of program information to interested research engineers and scientists. The organizational chart for the DOE Office of Energy Research (OER) on the next page delineates the six Divisions within the OER Office of Basic Energy Sciences (BES). Each BES Division administers basic, mission oriented research programs in the area indicated by its title. The BES Engineering Research Program is one such program; it is administered by the Engineering and Geosciences Division of BES. Dr. Oscar P. Manley is technical manager of the Engineering Research Program; inquiries concerning the program may be addressed to him, in writing or by phone at (301) 903-5822.

In preparing this report we asked the principal investigators to submit summaries for their projects that were specifically applicable to fiscal year 1991. The summaries received have been edited if necessary, but the press for timely publication made it impractical to have the investigators review and approve the summaries prior to publication. For more information about a given project, it is suggested that the investigators be contacted directly. 


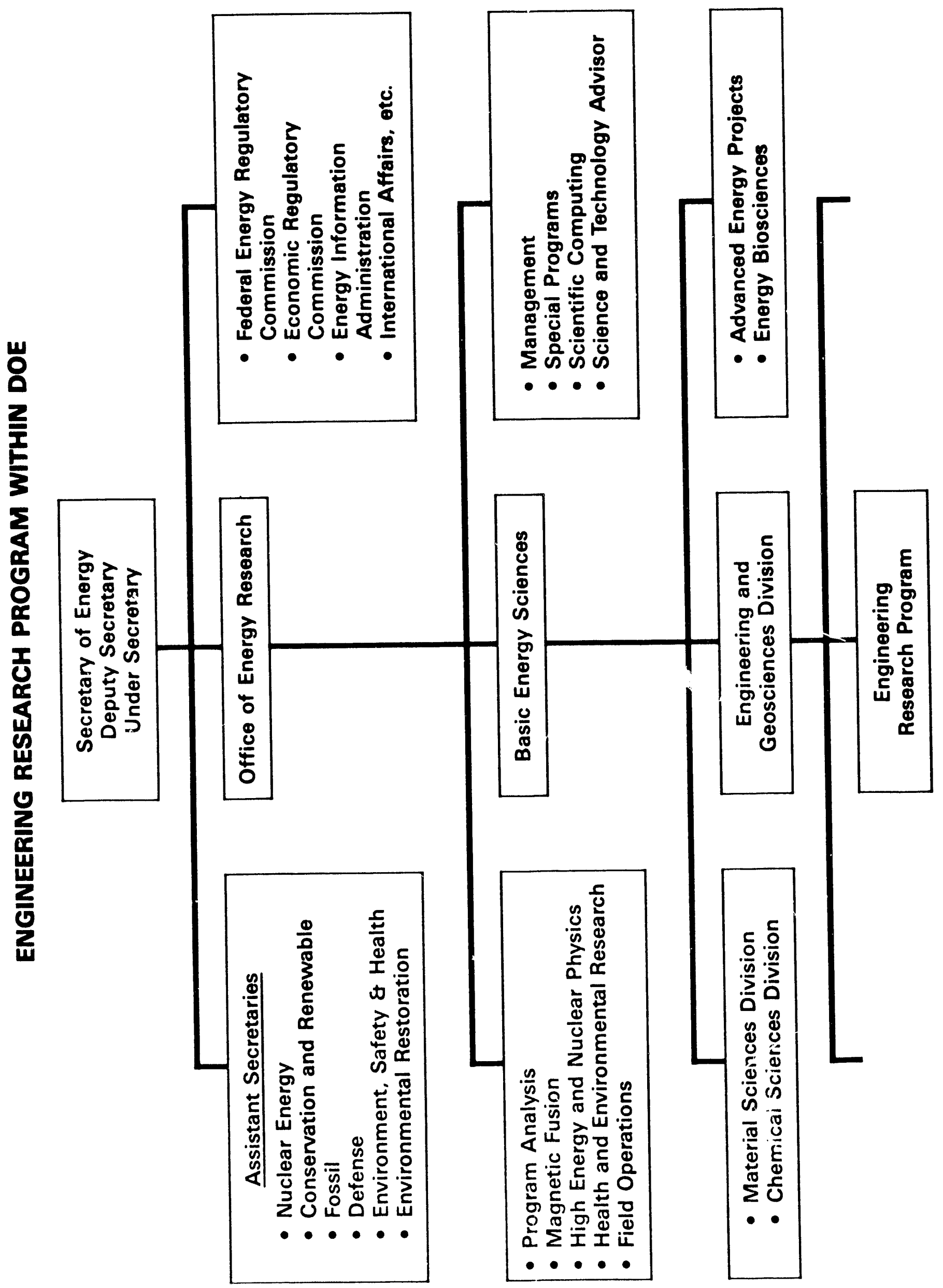




\section{Introduction}

The individual project summaries follow the program overview. The summaries are ordered alphabetically by name of institution and so the table of contents lists all the institutions at which projects were sponsored in fiscal year 1991.

The projects are numbered sequentially for individual identification in the indexes. Each project entry begins with an institutional-departmental heading. The names of investigators are listed immediately below the title. The funding level for fiscal year 1991 appears to the right of title; it is followed by the budget activity number (e.g.,01A). These numbers categorize the projects for budgetary purposes and the categories are described in the budget number index. The year in which either the project began or was renewed and the anticipated duration in years are indicated respectively by the first two and last digits of the sequence directly below the budget activity number (e.g., 90-3). The summary description of the project completes the entry. 


\section{Program Review BES Engineering Research}

The BES Engineering Research Program is one of the component research programs which collectively constitute the DOE Basic Energy Sciences Program. The DOE Basic Energy Sciences program supports energy related research in the physical and biological sciences, and in engineering. The chief purpose of the DOE Basic Energy Sciences Program is to provide the fundamental scientific base on which identification and development of future, national energy options will depend. The major product of the program becomes part of the body of data and knowledge upon which the applied energy technologies are founded; the product is knowledge relevant to energy exploration, production, conversion and use.

The BES Engineering Research Program was started in 1979 to help resolve the numerous serious engineering issues arising from efforts to meet U.S. energy needs. The program supports fundamental research on broad, generic topics in energy related engineering topics not as narrowly scoped as those addressed by the shorter term engineering research projects sponsored by the various DOE technology programs. Special emphasis is placed on projects which, if successfully concluded, will benefit more than one energy technology. During the first year several workshops were sponsored for the purpose of identifying energy related engineering research needs and initial priorities. Representatives from industry, academic institutions, national laboratories, and leading members of professional organizations (Engineering Societies Commission of Energy, American Society of Mechanical Engineers, Society of Automotive Engineers, and Joint Automation and Control Committee) participated in the workshops. In addition to the participants in the workshops, staff representatives from the DOE technology programs and other leading U.S. energy engineering experts made significant contributions to the setting of program priorities. There resulted from this process a strong confirmation of the need for a long range, fundamental enginecring research program with two major goals. The broad goals that were established by this process for the BES Engineering Rescarch Program are:

1) To extend the body of knowledge underiying current enginecring practice so as to create new options for enhancing energy savings and production, for prolonging useful equipment life, and for reducing costs without degradation of industrial production and performance quality; and

2) To broaden the technical and conceptual base for solving future engineering problems in the energy technologies. 
In this process, it was further established that to achieve these goals, the BES Engineering Research Program should address the following topics identified as essential to the progress of many energy technologies:

1) Advanced Industrial Technology: improvement of energy conversion and utilization, opening new technological possibilities, and improvement of energy systems.

2) Fluid Dynamics and Thermal Processes: broadening of understanding of heat transfer in nonsteady flows, methodology for reducing vibrations and noise in heat exchangers, and engineering aspects of combustion.

3) Solid Mechanics: continuum mechanics, fracture mechanics, thermomechanical bchavior in severe environments, aging \& lifetime reliability of structures.

4) Dynamics and Control of Processes and Systems: development and use of information describing system behavior (system models), performance criteria, and theorics of control optimization to achieve the best possible system performance subject to known constraints.

A Scoping Workshop held in December, 1985 confirmed the continued needs for research in these topical areas. Because of budgetary limitations, the implemented BES Engineering Research Program is somewhat less broad than the program envisioned above. At present, cqual emphasis is being placed in three carefully selected, high priority research areas; namely,

1) Mechanical Sciences including fluid mechanics (multiphase flow and turbulence) heat transfer, and solid mechanics (continuum mechanics and fracture mechanics).

2) System Sciences including process control and instrumentation.

3) Engineering Analysis including nonlinear dynamics, data bases for thermophysical properties of fluids, modeling of combustion processes for engineering application, and foundations of bioprocessing of fuels and energy related wastes.

These areas contain the most critical elements of the four topics enumerated above; as such they are of importance to energy technologies both in the short and long term, and therefore of immediate programmatic interest. It should be noted that other areas of basic research important to engineering are monitored elsewhere in BES. For instance, separation sciences and research on thermophysical properties are among the responsibilities of the Chemical Sciences Division, while microscopic aspects of fracture mechanics are in the domain of the Material Sciences Division. As resources permit, other high priority areas are being added to the Engincering Research Program. Thus, as a result of previous growth in the prograin budget an important development took place in the Engineering Research Program: two major concentrations of research were initiated. 
First, a new program was organized at Oak Ridge National Lab dealing with intelligent machines in an unstructured environment. Some resources are available for coordinated, more narrowly focussed, related, high quality research at universities and other research centers. All such activities are supported and administered directly by the Engineering Research Program, but some coordination of efforts with the ORNL program may frove useful.

Secondly in FY 1985, a collaborative research effort was started between MIT and Idaho National Engineering Lab. At present, the collaboration is in three distinct areas: Plasma Process Engineering, Automated Welding, and Fracture Mechanics. Collateral, high quality research efforts at other institutions are supported by the Engineering Research Program.

In the expectation of a future modest growth of this Program, two International Workshops on Two Fhase Flow Fundamental were held one in September 1985 and the other in March, 1987. The meetings were used to identify basic research needs in the field of two phase flow and heat transfer; summary reports of the workshops are available from the Program Office. The proceedings of the two workshops have been published as volumes in the series "Advances in Heat and Mass Transfer" (Hemisphere Publishing Company)

Two additional workshops were held during 1988. The first dealt with possible research opportunities in the field of novel devices using the new high temperature superconductors. The second addressed research needs for bioprocessing of fuels and energy related wastes. Reports of both workshops have been published. Additional funds have been provided in FY 1992 to initiate research in the above mentioned bioprocessing area. Of interest are relevant studies at the intersection of biology, biochemistry, and chemical engineering.

It should be mentioned too, that some very limited support is available for research on large scale systems. A report of a workshop on needs, opportunities, and options in this fịcld is available from Professor G.L. Thompson, Graduate School of Industrial Administration, Carnegie-Mellon University, Pittsburgh, PA 15213.

Research projects sponsored by the BES Engineering Research Program are currently underway at universities, private sector laboratories, and DOE national laboratories. In fiscal year 1991 the available program operating funds available amounted to about $\$ 14.8$ million. The distribution of these funds among various institutions and by topical area is illustrated on the next page. Project funding levels are mostly in the range of $\$ 50,000$ to $\$ 150,000$ per year. Typical duration of a project is three to four years, with some projects expected to last as long as ten years or more. The BES Engineering Res'arch projects stem almost without exception from proposais for competitive grants. Proposals which anticipate definite results in less than two years are usually referred to the appropriate DOE technology program for consideration. All those interested in submitting a proposal are 
encouraged to discuss their ideas with the technical program manager prior to submission of a formal proposal. Such discussion helps to establish whether or not a potential project has a reasonable chance of being funded. The primary considerations for possible support are the technical quality of the proposal and the professional standing of the principal investigators and staff. An effort is made to attract first rate, younger research engineers and energy oriented applied scientists. A high technical caliber of research is maintained by requiring that the projects supported have potential for a significant contribution to energy related engineering science, or for an initial contribution to a new energy relevant tcchnology. Sponsored projects are selected primarily for their relevance to DOE mission requirements; the contribution to energy related higher education is an important but secondary consideration. Thus projects sponsored at universities are essentially limited to advanced theoretical and experimental studies usually performed by faculty members, staff research scientists, and doctoral candidates. 


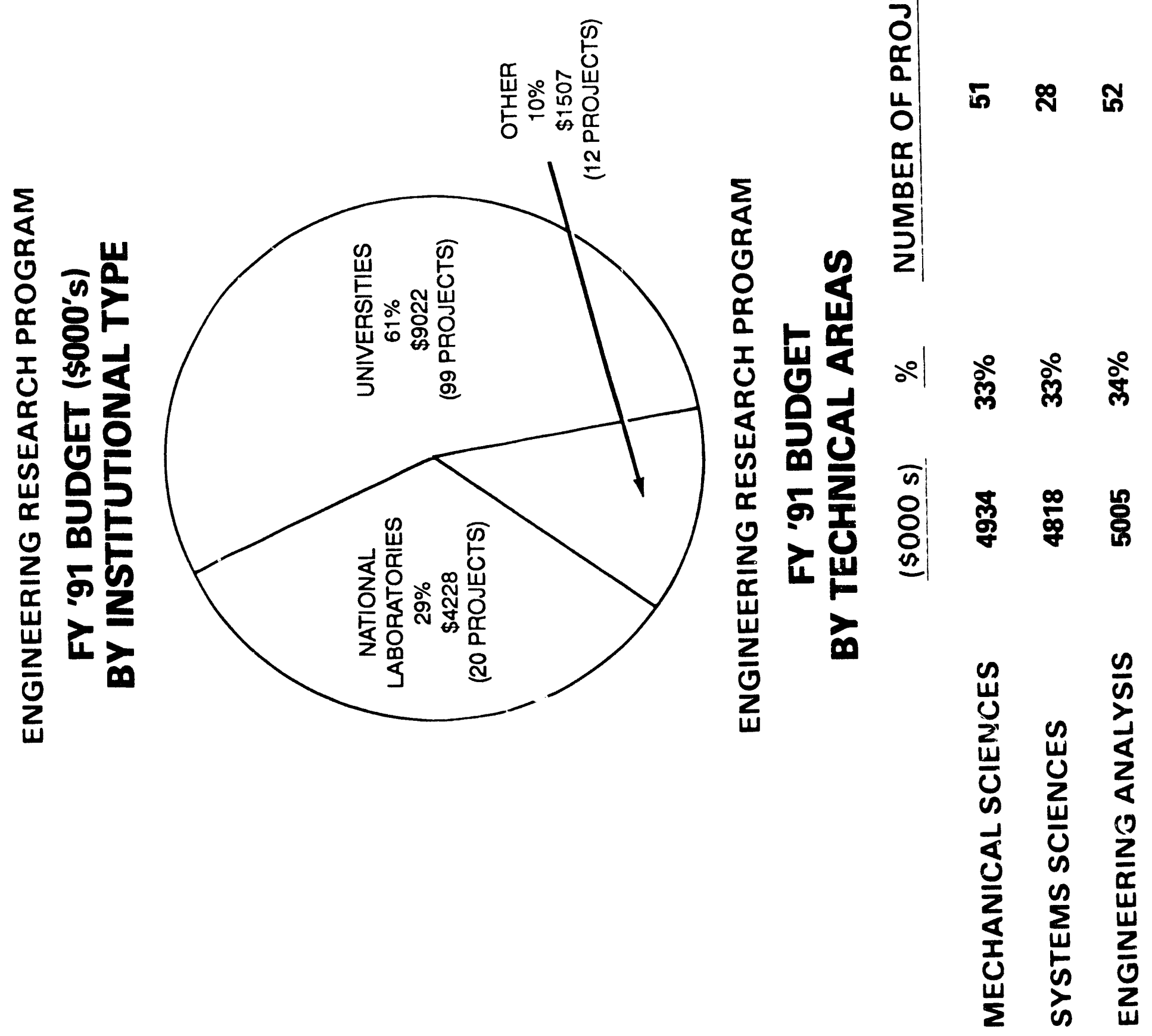




\section{Table of Contents}

University of Alabama . . . . . . . . . . . . . . . 1

Argonne National Laboratory . . . . . . . . . . . . . . . . . . . . 1

Arizona State University . . . . . . . . . . . . . . . . 2

Battelle Memorial institute . . . . . . . . . . . . . . . 3

Boston University . . . . . . . . . . . . . . . . . . . 3

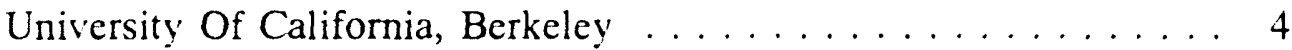

University Of California, Los Angeles ................ 4

University Of California, San Diego ............... 5

University Of California, Santa Barbara . . . . . . . . . . . . 8

California Institute Of Technology . . . . . . . . . . . . . . 9

Carnegie Mellon University . . . . . . . . . . . . . . . 9

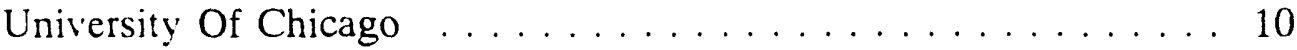

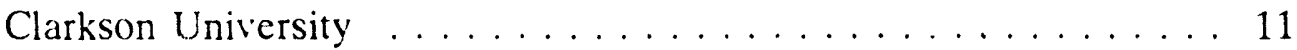

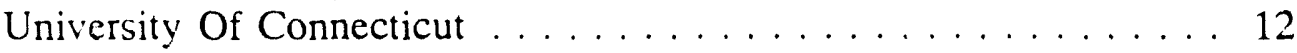

Cornell University . . . . . . . . . . . . . . . . . . . 12

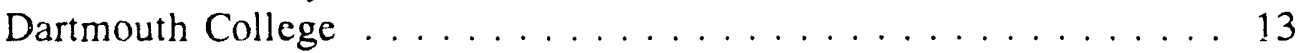

Duke University . . . . . . . . . . . . . . . . . . . . 14

Engineering Science Software, Inc. . . . . . . . . . . . . 14

University of Houston . . . . . . . . . . . . . . . . 15

Idaho National Engineering Laboratory . . . . . . . . . . . . . . . . 15

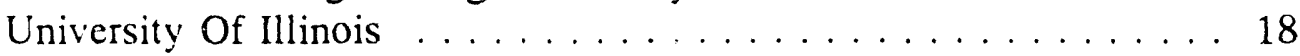

University of Illinois At Chicago . . . . . . . . . . . . . . . 19

Jet Propulsion Laboratory . . . . . . . . . . . . . . . . . . . . . . 19

Johns Hopkins University . . . . . . . . . . . . . . . . . . . 20

Robert H. Kraichnan, Inc. . . . . . . . . . . . . . . . . . . 20

Lawrence Berkeley Laboratory . . . . . . . . . . . . . . . . . . . . . 20

Lovelace Medical Foundation . . . . . . . . . . . . . . . . . . . 21

University of Maryland . . . . . . . . . . . . . . . . . 22

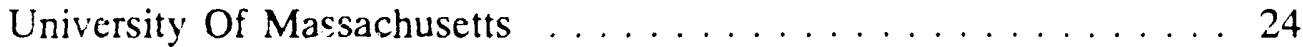

University of Massachusetts-Lowell . . . . . . . . . . . . . . . 24

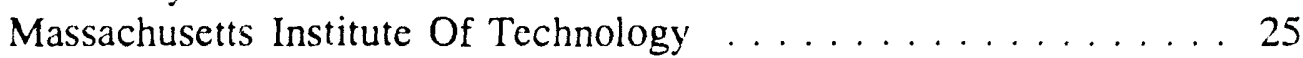

University of Minnesota . . . . . . . . . . . . . . . . . . . . . . . 29

National Institute Of Standards And Technology . . . . . . . . . . 30

The City University Of New York, The City College . . . . . . . . . 32

North Carolina State University . . . . . . . . . . . . . . . . 33

Northwestern University . . . . . . . . . . . . . . . . . 33

University of Notre Dame . . . . . . . . . . . . . . . . 35 


\section{Table of Contents (continued)}

Oak Ridge National Laboratory . . . . . . . . . . . . . 35

Ohio State University . . . . . . . . . . . . . . . . . 36

Oregon State University . . . . . . . . . . . . . . . 37

Pacific Northwest Laboratory . . . . . . . . . . . . . . . . . . . . . 37

University Of Pennsylvania . . . . . . . . . . . . . . . . 37

Physical Optics Corporation . . . . . . . . . . . . . . . 38

Physical Sciences Inc. . . . . . . . . . . . . . . . . 38

Princeton University $\ldots \ldots \ldots \ldots$

Purdue University . . . . . . . . . . . . . . . . . . . . . . . . . . 39

Rensselaer Polytechnic Institute . . . . . . . . . . . . . . . 40

Rice University . . . . . . . . . . . . . . . . . . . . . 42

University of Rochester . . . . . . . . . . . . . . . . . . 42

Rockefeller University . . . . . . . . . . . . . . . . . . . . . . 43

Sandia National Laboratories . . . . . . . . . . . . . . . . . . . . 43

University Of Southern California . . . . . . . . . . . . . . . . . 44

Southwest Research Institute . . . . . . . . . . . . . . . 45

Stanford University . . . . . . . . . . . . . . . . . . . . . . . 45

Stevens Institute of Technology . . . . . . . . . . . . . . . 49

Syracuse University . . . . . . . . . . . . . . . . . . . . 49

University Of Texas At Austin . . . . . . . . . . . . . . . 49

Tufts University . . . . . . . . . . . . . . . . . . 50

United Technologies Research Center . . . . . . . . . . . . 50

Nashington University . . . . . . . . . . . . . . . . 51

Washington State University $\ldots \ldots \ldots \ldots \ldots$

University of Wisconsin $\ldots \ldots \ldots \ldots 2$ 
University Of Alabama

Dept of Mathematics

Tuscaloosa, AL 35487

$\$ 52,068$

$01-\mathrm{C}$

91-3

Hydrodynamic Instabilities and Coherent

Structures

\section{A. Frenkel}

The objective of this project is to advance the understanding of instabilities of several fluid systems relevant to energy engineering sciences. Sorne problems, approaches to their solution, and results follow.

For the problem of coherent structures and the inverse energy cascade in turbulent flows, both statistical ensembles and deterministic periodic flows are considered.

It is found that an isotropic turbulent flow is stable to the mean-flow perturbations. This suggests that some statistical anisotropy is necessary for the growth of the coherent structures.

The same techniques, based on mathematicai insights into the properties of functionals of Gaussian processes, yields a new description of the diffusion of a passive scalar - such as the temperature - in a turbulent flow.

For a deterministic unidirectional flow with a sinusoidal profile of velocity, it is shown rigorously - by using continued fractions - that some perturbations which do not have the same periodicity as the basic flow, can nevertheless grow. The much more complicated case when the flow is, in addition, sinusoidal in time is reduced to an infinite algebraic eigenvalue problem. Its Galerkin approxirnation results are not restricted to large scales, in contrast to earlier results. An exact solution of a generalized Orr-Sommerfeld equation in the inviscid case is found and compared to the Galerkin-based results.

For the core-annuiar flow - important in the lubricated pipelining of heavy oils - a new nonlinear interface equation is derived. It reflects the dynamics of the core in addition to the dynamics of the lubricating film. Several domains of basic parameters are indicated in which the instability cannot break up the flow. The finite but small oscillations of the interface can have the character of either deterministic chaos or else a nonlinear propagating nave.

\section{Argonne National Laboratory Materials \& Components Tech Dlv $\$ 144,000$ Argonne, IL 60439 \\ $01-A$}

Chaos in Fluid-Structure Systems S. Chen, T. Mulcahy

Integrated theoretical and experimental studies are being performed to enhance the understanding of nonlinear oscillations and dynamic instability phenomena involving both fluids and solid structures and their coupling. The objectives are to contribute to the explanation of observed phenomena, providing insights into chaotic characteristics of such coupled mechanical systems and ultimately, to the solution of engineering design problems. This is a joint project with Professor F. Moon at Cornell University.

Fluidelastic instability of loosely supported tubes, vibrating in a tube support plate-inactive mode, is suspected to be one of the main causes of tube failure in some operating steam generators and heat exchangers. As a vehicle to understand the nonlinear behavior of fluid-structure systems, fluidelastic instability of loosely supported tube rows in crossflow is being studied in detail. Tube row with a motion-limiting stop configuration will be tested to investigate various response characteristics and a mathematical model tased on the unsteady flow theory will be developed to predict the response characteristics of this classical fluid-structure system.

Energy systems have had a history of dynamic structural instabilities caused by fluid flow resulting in costly component repair and replacement and loss of anergy production. The understanding of dynamic characteristics, including periodic oscillations, random vibration, and chaotic motion, will impact the design and reliability of these systems in both the near and long term. In addition, fluid-structure systems are classical examples of autonomous systems that can exhibit chaotic behavior. Knowledge of the fluid-structure systems may provide some insights into the craotic behavior of other energy system components. 


\section{Argonne National Laboratory Materials \& Components Tech Div $\$ 132,000$ Argonne, IL 60439 01-A \\ Bounds on Dynamic Plastic Deformation C. Youngdahl}

Analytical studies are being performed to develop methods for approximating or bounding the dynamic plastic deformation of structures. In many applications where the load is transmitted to the structure through a fluid, details of the load history and spatial distribution significantly affect the final plastic deformation. The objective of the program is to devise mode approximation methods and lcad correlation parameters which can be used to predict the final deformed shape and characterize the effects of the load without "sorting to detailed numerical analyses. These approximation methods have three important uses: to perform design and safety analyses of structures over a wide range of design variables and loadings; to validate computer programs which have a nonlinear dynamic plasticity capability; and to correlate experimental simulations with actual or predicted events.

The dynamic plastic deformation of some basic structural configurations are being analyzed for loadings which vary both in magnitude and region of application with time. Mode approximation methods and load correlation parameters are hypothesized and their usefulness in predicting final plastic deformation is determined. The methoos, based initially on a rigid, perfectly plastic material model and small deformation response, are extended to include strain hardening, an initial elastic lesponse period, and large deformation interactions.

\section{Arizona State University Mechanical \& Aerospace Eng Tempe, AZ 85287}

$\$ 55,000$

01-A

$88-4$

\section{Coritinuum Damage Mechanics II D. Krajcinovlc}

The efforts in the first two years of this research program were focused on the development of a micromechanically inspired continuum damage mechanics model. Specifically, the objective was to formulate a rigorous micro-to-macro transition along with the conditions which must be satisfied for this transition to be valid. For example, it was demonstrated that the change in elastic compliance is a proper choice for the thermodynamic macro-flux since it represents an orientation weighted volume average of microfluxes. The corresponding affinity is the energy release rate integrated along the crack perimeter and averaged across its surface. The macropotential is subsequently determined as an inner envelope of the micro-potential. The corresponding damage surface is a convex piecewise smooth hypersurface possessing the normality property. The ensuing cornputations clearly indicate the simplicity and accuracy of the proposed model in several illustrative examples.

The micro-to-macro transition is, however, proven to be valid only as long as the application of the effective continua theories is justified. In other words, the macro flow potential cannot be proven to exist when the influence of the direct interaction of micro-defects on the deformation becomes significant.

One of the most important aspects of the analyses of brittle deformation processes is the determination of failure which is, obviously, associated with a critical microcrack density. However, the analytical modeling of the so-called cooperative phenomena, characterized in the considered case by a disorder of the material microstructure and the interaction of many defects of random geometry, distribution and orientation represents a serious problem. This class of problems, being inherently nondeterministic in nature, is seldom if ever addressed in continuum mechanics. A promising strategy, to be explored in the continuation of this research program, is to consider the application of the so-called percolation theory. One of the two main problems is to make a transition between the lattice and continuum percolation theory and recover the damage micro-flux (Budiansky-O'Connell variable) and enable mapping between the two models. The other problem is that the disorder is introduced into the percolation model traditionally by a bimodal distribution which might be to restrictive for real materials having a richer hierarchy of strengths on the micro-scale. Nevertheless, it is felt that the percolation theory offers a viable framework for the studies of the brittle deformation processes in the vicinity of the percolation thresholds (which are different that those associated with transport phenomena). The application of the percolation theory is important 
since it enables studies of estimates which the local fluctuations of stresses (hot-spots) and strengths (weak-links) have on the failure of the material.

\section{Battelle Memorial Institute Mechanics Dept Columbus, OH 43201-2693}

An Investigation of the Effects of History Dependent Damage In Time Dependent Fracture Mechanics

\section{F. Brust}

The demands for structural systems to perform reliably under severe operating conditions continue to increase. Modern energy production facilities experience degradation and damage because they operate in a severe high-temperature environment where time dependent siraining and damage may lead to structural failures. The goal of this research is to study the high temperature damage and failure processes and to further develop a method for predicting this behavior in an effort to increase structural life. in particular, we focus on time dependent damage which occurs under history-dependent loading conditions, i.e. transient conditions.

The types of time dependent (creep) damage considered in this program include: sustained load creep, variable load creep, and variable load creep with thermal gradients. During the first year of this study, the implications of using Norton's creep law on various integral parameters used to characterize crack tip phenomena were evaluated as a function of time. Other constitutive laws for time dependent materials such as those of Murakami and Ohno are being implemented into the finite element code. In addition, constitutive property data and high temperature creef crack growth data are being obtained on stainless steel. These experiments will be used to verify analytical predictions and characterize time and history dependent damage during crack nucleation a:ıd growth.

The results from this work will be used by practicing engineers to enhance the life of high temperature structural systems during the design phase.

\section{Boston University Department of Chemistry Boston, MA 02215}

Transport in Porous/Disordered Materials T. Keyes

The aim of this project is the construction of theories of the transport processes-diffusion, fluid flow, electromagnetic wave propagation, etc. in materials where strong interactions or disorder cause a near stoppage of the transport. Of special interest are materials with percolation thresholds, where the disorder blocks transport altogether, and systems near a glass transition. The research is broadly based and a wide range of theoretica, and computational techniques are employed. Progress along three fronts was made in the past year.

Harmonic normal mode analysis is routinely applied to stable solids, but is not normally considered useful for fluids. We have developed a new method for fluid dynamics, based upon normal mode analysis, in which transport is governed by the unstable modes of the system. The method worked extremely well for supercooled liquids and is now being applied to suspensions of spheres.

Glass ceramics are composed of irregular crystallites in a glassy matrix. The propagation of light through these disordered materials was studied. The structure of the crystallites was related to the properties of the scattered light in a completely new scheme which requires the intensity statistics, but no knowledge of optical properties.

High intensity light will break chemical bonds and cut material. A fairly comprehensive model of this very nonlinear process was constructed and soived, with qualitative agreement with several observations. Computer simulation of the phenomenon was begun, with an eye to the study of laser-induced shock waves and to sorting out the "energy bookkeeping" -the ultimate destinations of the laser energy. 


\section{University Of California/B \\ Dept of Electrical Engineering \\ \& Computer Sciences \\ Berkeley, CA 94720}
$\$ 93,300$
06-C
90-3

\section{Self-Generated Stochastic Heating in an RF Discharge}

A. Lichtenberg, M. Lieberman

The purpose of this project is to study electron heating mechanisms in radio frequency (r.f.) discharges. These discharges are used extensively by industry for surface modification of electronic and mechanical materials. In addition to the usual r.f. ohmic heating in the plasma interior, it has been found theoretically that stochastic heating at the plasma surface plays a major role. The stochastic electron heating arises from successive decorrelated reflections of electrons with the oscillating sheath near the surface of the discharge. Power is also transferred directly to the ions by acceleration in the sheaths. The efficiency of stochastic heating depends on the detailed sheath dynamics. For a low pressure discharge at a pressure of 3 mTorr, power of 100 watts, and frequency of $13 \mathrm{MHz}, 95 \%$ of the electron heating is predicted to occur stochastically.

The sheath dynamics are being measured and related to the predicted stochastic heating for a plane parallel argon discharge. Depending on the details of the sheath motion, the enhancement factor of the stochastic heating can change, and consequently so can the ratio of electron to ion power at a given overall power level. The measured results, together with measurements of other plasma parameters, will be used to construct better predictive models of discharge behavior and parameter scaling.

Stochastic heating can be enhanced with a resonant helical discharge structure. This geometry is of importance for materials processing applications and is under investigation as part of the overall program to understand plasma heating and develop models of r.f. processing discharges.

\section{University Of California/LA Mech, Aero \& Nuclear Eng Dept \\ $\$ 78,790$ 01-C School of Eng \& Applied Science 89-3}

\section{Basic Studies of Transport Processes in Porous Media \\ I. Catton}

The research covers two broad areas: 1) single-phase convection in porous media, and 2) two-phase convection in porous media. The objective of this study is to develop physical understanding of the governing nhenomena and models for prediction of transport piucesses by theoretical and experimental means.

Non-Darcian forced convection in a porous medium with anisotropic dispersion was studied. Convective heat transfer in a packed tube was modeled. It was shown that heat transfer in the thermally developing region is affected by axial dispersion when the Peclet number is less than 10. The effects of hydrodynainic dispersion on the corvection onset of a porous layer with volumetric heating were shown to reduce the critical Rayleigh number.

Generalized models have been developed for turbulent transport processes in highly permeable porous media with Reynolds number greater than orie hundred. Improved transfer equations for the solid and fluid phases were obtained by averaging ine turbulent transfer equations over a heterogeneous medium. A series of two-dimensional turbulent flow, energy, and mass transfer models was developed. The results of the modeling were compared with the theoretical and experimental work of others. A close connection between closure of heat and mass transfer models, and boundary conditions is shown by taking proper account of surface roughness within and on the boundaries of a porous medium.

Steam injection into water flow was studied analytically and experimentally. For slow flow (Darcy flow), the analysis yielded values of all important parameters that compare well with the experimental data. The importance of dispersion to the transport of energy at the steam-water interface was clearly demonstrated. At high steam injection rates, the steam-bubble boundary was shown to be unstable. 
$\begin{array}{lr}\text { University Of California/LA } \\ \text { Mech, Aero \& Nuc Eng Dept } & \$ 87,654 \\ \text { School of Eng \& Applied Sclence } & 06-\text { C } \\ \text { Los Angeles, CA 90024-1597 } & 89-3\end{array}$

Energy and Particle Transport In Random

Media

G. Pomraning

The goal in this research is to develop a comprehensive theory of linear transport/kinetic theory in a stochastic mixture of solids and immiscible fluids. Such a theory should predict the ensemble average and higher moments, such as the variance, of the particle or energy density described by the underlying transport/kinetic equation. The statistics to be studied correspond to $\mathrm{N}$-state discrete random variables for the interaction coefficients and sources, with $N$ denoting the number of components in the mixture. The mixing statistics to be considered are Markovian as well as more general statistics.

In the absence of time dependence and scattering, the theory is well developed and described by the master (Liouville) equation for Markovian mixing, and by renewal equations for non-Markovian mixing. The intent of further work is to generalize these treatments to include both time dependence and scattering. A further goal of this research is to develop aporoximate, but simpler, models from the comprehensive theory. In particular, a specific goal is to formulate a renormalized transport/kinetic theory of the usual nonstochastic form, but with effective interaction coefficients and sources to account for the stochastic nature of the problem. Numerical comparisons of all models will be made against Monte Carlo simulations which involve a straightforward average of solutions for a large number of physical realizations of the statistical mixing. Extensions to nonlinear kinetic equations will also be investigated.

\section{University Of California/LA}

Physics Dept

Los Angeles, CA 90024

$\$ 115,920$

$06-C$

$90-3$

Wave Turbulence and Self-Localization in Continuous Media

S. Putterman

The fate of energy which is injected into fluids that are far from equilibrium is being investigated from the experimental, theoretical and computational point of view. These off-eq:dilibrium systems can display a tendency to a) form s! juctures b) develop a stochastic spectrum of energy and c) focus energy. Regarding a) the formation of solitons, domain walls and kinks in continuous media is being studied. Regarding b) the tendency of a spectrum of acoustic motion to become wave turbulent is being investigated and regarding $c$ ) the transduction of round into lift is being measured. This last phenomenon results from a self-focusing instability that concentrates energy by a factor of one trillion.

\section{University of California/SD \\ Scripps Inst. of Oceanography La Jolla, CA 92093-0402}

Signal Processing in Chaos

90-4

\section{H. Abarbanel}

We are investigating methods for modeling fluid flows and plasma dynamics of relevance to energy problems when these physical phenomena exhibit motion on fractal objects called strange attractors. In these cases, which are quite common both in the settings indicated, and in electrical circuits and condensed matter systems, the time series have continuous broadband power spectra and quite irregular behavior. We are engaged in methods for identifying the space in which to work for these systems (phase space reconstruction), methods for computing the invariants of the motions, and methods for making physical models for prediction in these irregular systems. These systems are inherently less predictable than familiar linear systems, but their predictability is nonzero and may be extracted in a systematic fashion from the time traces themselves.

The work in FY91 has concentrated on methods to extract chaotic, deterministic signals from noisy measurements. The noise may be due to environmental conditions or measurement errors or may have been imposed on purpose to mask the signal. With these "cleaning" tools, we will clean up laboratory and field data for use in the other parts of the signal processing effort. 


\section{University Of California/SD} Dept of App Mech \& Eng Scl La Jolla, CA 92093

$\$ 161,680$

06-B

$90-3$

Experimental and Theoretical Study of Fuel Droplets Subject to Straining Flow $P$. Libby, F. WIIllams

This research involves a combined experimiental and theoretical effort related to the behavior of fuel droplets. The initial experiments concerned the determination by photographic means of the trajectories and under the circumstances involving mass loss, of the radius histories of individual droplets in well defined, nonuniform laminar streams. The original studies involved a nonpremixed laminar flame in a counterflow configuration, isotherrial counterflowing nitrogen streains and a vertical Poiseuille flow. The results of these studies have appeared in Combustion Science and Technology. Recently these same techniques have been applied to a droplet on a vertical trajectory entering the horizontal flow from a jet with close to a top-hat velocity distribution. The purpose of this experiment was to elucidate the source of the lift found in the early experiments. However, the results, which are to appear in Combustion Science and Technolegy establish that conventional force laws accurately predict the droplet trajectories with the consequence that the source of the lift measured in the earlier configurations remains obscure. Our current research concerns fuel sprays in counterflowing streams which result in flat nonpremixed flames. Measurements of the velocity distribution are underway and in due course the droplet size and velocity will be measured. Complementary theoretical research is underway.

\section{University Of California/SD}

Department of Chemistry, 0340

La Jolla, CA 92093

\section{Noisy Nonlinear Systems \\ K. Lindenberg}

Transport and reactions on fractals occur in many natural phenomena including geological processes and processes in the solid state or on surfaces. Much that is known comes from computer simulations. Our efforts have been directed toward gaining a theoretical understanding of these processes.
The calculation of the time that it takes to reach a given point on a fractal is relevant for events that are triggered when an excitation or molecule or charge reaches a designated point. Our analysis is based on a renormalization approach that yields exact results on some deterministic fractais. Whether these methods can be applied to random fractals is still an open question; we have applied them to structures that mimic random fractals.

The rates of even the simplest diffusion-controlled reactions in low-dimensional systems behave very differently than the standard law-of-mass-action descriptions and have been called "anomalous" and indicative of "mathematical poisoning." Our calculation of reaction rates is based on scaling arguments that lead to parameterized results. Even this limited success has provided a great deal of insight.

We continue to make progress in the (unrelated) problem of the effect of colored noise on the evolution of a system. We have obtained analytic results for a dye laser driven by Gaussian fluctuations and also for a bistable system driven by dichotomous noise.

\section{University Of California/SD Pure \& Applied Physical Science $\$ \$ 94,000$ La Jolla, CA 92093 06-C \\ 91-2 \\ Knot Invariants and the Thermodynamics of Lattice Gas Automata D. Meyer}

Lattice gases, operating as cellular automata and amendable to massive parallelism, which simulate the Navier-Stokes equation in two arid three dimensions, were introduced in 1985 by the principal investigators (Phys. Rev. Lett). Such lattice gases are formed of "Boolean molecules" with discrete time, space, and velocity. Their collision laws are designed to conserve particle number and momentum. Macroscopic momentum averages satisfy the incompressible Navier-Stokes equation, in a suitable physical limit. Groups in the United States and in France are already engaged in theory, simulations, and hardware projects for lattice gas hydrodynamics. Preliminary results indicate that the method is easy to implement (with or without boundaries), robust, and reproduces known hydrodynamic phenomena. It may eventually yieid a new simulation strategy 
for complex turbulent flows, with many practical applications.

It was proposed that the lattice gas method be evaluated, and that modified models with higher Reynolds numbers be studied. Two-dimensional computer models were created to run on the CRAY - XMP, the CRAY II, and on the Cons ection Machine II (a computer with 65,000 sentral processors). These models ran at the ra of $80,000,000,10,000,000$, and $2,000,000,000$ cell updates per second, respectively. Comparisons with other calculational methods have been done. A three-din'ensional program has been developed which produced the highest Reynolds numbers attainable with 24 bits required per cell. Flow past a square plate has been successfully modeled by this code. Summaries of this and other recent work has been published in several articles in the August issue of Complex Systems. Lattice gas methods for solving partial differential equations appear useful and promising. Expectations of their capabilities on future machiries are now being estimated.

\section{University Of California/SD} Inst for Nonlinear Science, R-002 $\$ 89,545$ La Jolla, CA 92093

Formation of Three-Dimensional Singularities, Regularization Procedures and Markovian-Multifractal Description of Turbulent Flows

\section{E. Novikov}

The study of intermittent turbulent flows with high Reynolds number, based on development and unification of different complementary approaches, is proposed. The new recently developed method is based on conditional averaging of equations for a local characteristic of motion, which has a mechanism of self-amplification. An exact closed equation for a conditionally averaged threedimensional vorticity field (with fixed vorticity at a certain point) is obtained from the Navier-Stokes equation. Corresponding closed equation is derived for conditionally averaged vorticity gradient in two-dimensional turbulence. Solutions of these equations are obtained. The high order two-point moments of vorticity and vorticity gradient are presented. The obtained results can be verified by laboratory and field measurements, as well as in numerical experiments. The developed method is

quite general and can be applied to a variety of physical systems with strong interaction, including magnetized plasma.

\section{University Of California/SD}

Dept of Physics

La Jolla, CA 92093

$\$ 211,797$

$01-B$

90-3

Traveling-Wave Convection In Fluid Mixtures C. Surko

While some progress has been achieved recently in the understanding of the dynamics of free convection occurring in single component fluids, much less is known about the phenomenology of convection in fluid mixtures. Yet in realistic situations it is the latter that play the important role. Examples of interest to engineering include stability of double-diffusive systems, e.g. solar ponds, solidification of metal alloy casts, convection in fuel droplets, and so on.

The proposed research addresses the interaction of traveling waves with convective motion in a binary fluid mixture. Preliminary results show that in a relatively simple experiment the behavior of the system is such that on varying one or two control parameters the whole gamut of states ranging from a laminar traveling-wave state to well developed turbulence can be traversed. Specifically the experimental setup consists of an annular channel with a large radius. In it a waterethanol mixture is heated from below giving rise to free convection. However, under certain conditions, in addition to this expected phenomenon, there occur traveling-waves interacting in complicated ways with the patterns driven by natural convection. The resulting oscillatory convection seems to be prototypical of sirnilar phenomena arising in many contexts, ranging from oceanic motion to instabilities in liquid crystals.

The proposed research will address three related topics: 1) weakly nonlinear traveling waves in one dimension; 2) nonlinear pulses in an annulus; and 3) measurement of concentration field. 


\section{University Of California/SB}

Dept of Physics

Santa Barbara, CA 93106

$\$ 125,840$

06-C

90-3

Bifurcations and Fatterns in Nonlinear

Dissipative Systems

G. Ahlers, D. Cannell

This project consists of experimental investigations of non-linear non-equilibrium fluid-mechanical systems, with an emphasis on heat transport, pattern formation, and bifurcation phenomena. These issues are being studied in Rayleigh-Benard convection, using both pure and multi-component fluids. They play an important role in such energy-related issues as crystal growth from a melt with and without impurities, the catastrophic inversion of salt lakes such as the Dead Sea, energy production in solar ponds, and various oceanographic phenomena.

The work utilizes computer-enhanced shadowgraph imaging to visualize the convective flow patterns. The technique can detect the flow field even when the convection ihreshold is exceeded by only $0.1 \%$. Ir parallel, high-resolution heat-flux measurements are made with a resolution of $0.05 \%$. Thus, the relationship between the pattern and the heat transport san be studied in great detail.

In pure fiuids, we are investigating the mechanisms for the convective oiset. In most physical systems the flow is initiated by properties of the experimental cell which lead to an imperfect bifurcation. Fiecently it has been possible, however, to perfect the apparatus to a point where stochastic effects control the evolution of the flow.

Beyond the convective threshold we are interested in the evolution and stability of various convective patterns in containers with simple sidewall geometries. We expect that our results will help in the development of theoretical models for pattern stability.

A particularly interesting pattern-stability problem occurs in binary-mixture convection. For certain values of the parameters of this system, there exist spatially localized patches of travelling waves of convective rolls. The existence of these states cannot be explained by the usual models with relaxational dynamics, but rather seem to require non-potential theories. We are studying quantitatively the wavenumber, frequency, amplitude, and spatial extent of these localized states. This information will permit a distinction between several competing theoretical models.

\section{University Of California/SB}

Dept of Chemical \& Nuc Eng

Santa Barbara, CA 93106

$\$ 197,725$

$01-\mathrm{C}$

91-3

Turbulence Structure and Transport

Processes in Wavy Liquid Streams

S. Banerjee, G. Hetsroni

This project is aimed at studying basic turbulence structure in a liquid which flows in a horizontal flume. The experimental techniques used in this study include a three-dimensional Laser-Doppler velocimeter, with a $50 \mathrm{~m}$ measuring volume; and an oxygen bubble tracing technique with high speed videos and flash photography.

The oxygen bubble technique resulted in capturing of the dntailed turbulence production at the solid wall and at the liquid-air interface. The details of the high speed-low speed velocity streaks at the wall, the coherent structures near the wall, and the turbulent bursts were visualized and measured. It was found that the iurbulent coherent structures reach the interface and are visualized there as patches. These patches then have a profound effect on the heat and mass transfer at the interface.

High speed and low speed velocity streaks were also observed at the liquid-gas interface, when shear was applied on the liquid by the gas flowing cocurrent or countercurrent to the liquid. The coherent structures from the interface were then observed to be ejected downwards.

Waves, which were generated by a wave generating machine, were otserved to have a profound effect on the frequency of ejections of the coherent structures at the wall. The effects of the wave amplitude and wave length on the frequency and strength of the turbulent bursts are now being analyzed.

These experiments were complemented by numerical computations. The full Navier-Stokes equations were solved by a pseudospectral method to provide quantities which are difficult to obtain by flow visualization and single-point 
velocity measurements. The numerical computations were shown to capture the basic physics which is associated with the turbulence generation due to shear.

The three-dimensional Laser-Doppler velocimeter was assembled, and the hardware and software were readied for the measurements. The measurements with the LDV will be complemented by visualization by means of the oxygen bubbles and high speed video.

\section{California Institute of Technology Chemical Engineering 210-41 Pasadena, CA 91125}

\section{Modeling for Process Control} M. Morarl

One key difficulty which stands in the way of application of many advanced control techniques in the chemical process industries is the need for a model to describe the dynamic ehavior of the process to be controlled. The sbjective of this research program is the development of a range of new modeling techniques, in particular: development of techniques for building physicsbased, low order, nonlinear models with physically meaningful parameters, specifically for the purpose of (robust) linear and nonlinear controller design; and development of methods for the identification of linear black box models and the associated uncertainty description suitable for robust control system design.

At present, use of the Partial Least Squares Technique (PLS) is explored to identify impulse response models for linear dynamic systems and an associated uncertainty description which is suitable for robust control system design. The resulting uncertainty descriptions are given in terms of orthogonal functions which in turn can be used directly for controller synthesis.

\section{Carnegie Mellon University}

Chemical Eng Dept \& Graduate

School of Indus Admin $\$ 152,603$

PIttsburgh, PA 15213

Integration of Redesign Methodologies for

Chemical Processes

L Biegler, I. Grossmann, G. Thompson, A.

Westerberg

Process redesign, commonly known as retrofit, is a major task in the chemical industries. However, most systematic design strategies have been developed for new process and often do not apply. This project addresses the development of systematic design methodologies for the redesign of chemical processes. This integrated approach deals with a broad range of problems in process redesign. Here we discuss progress in the following areas:

1) Development of Efficient Optimization Algorithms for Discrete and Continuous Variables. Efficient decomposition strategies for nonlinear programming have been developed for large-scale process optimization. These are currently being tailored to various problem types. The work also includes the solution of several large scale combinatorial optimization problems that frequently occur in chemical engineering applications, namely, travelling salesman, and set covering (and partitioning) problems. The previously devised algorithms have been developed on various sequential and parallel computers such as the GRAY YMP and the Butterfly.

2) Redesign of Multicomponent Separation Sequences. We have completed a study to retrofit well-behaved distillation problems and are now examining the retrofit of problems involving significant nonideality in component behavior. Such problems occur commonly in waste minimization problems. Our goal is provide a methodology for redesigning complex separation systems to reduce the levels of trace species which must then be handed over to waste treatment facilities. We are examining the potential for significant reduction in the use of energy to remove trace species by redesigning such processes. 
(3) Redesion of Energy Management Systems. Mixed-integer nonlinear programming (MINLP) models have been developed for grassroots and the redesign problems. The unique feature of these models is the fact that the level of energy recovery and selection of matches is optimized simultaneously without fixing temperature approaches. Work is under way to develop a branch and bound method for finding the global optimum in these problems. Also, an LP/NLP based branch and bound method is being developed to expedite the solution of the MINLP models. Finally, the interactive program SYNHEAT is under development for the implementation of these models.

(4) Redesign for Flexibility, Controllability, and Reliability. The expected stochastic flexibility, a new measure that integrates the two operability characteristics, has been developed. The proposed measure can be used to evaluate the probability of feasible operation given uncertainties in process parameters and operational states. Current work includes the efficient integration of multiple integrals, the handling of nonlinear process models, and bounding strategies that require the examination of only few states. The application of the expected stochastic flexibility to bi-criterion optimization problems for retrofits is also being considered.

(5) Optimization-Based Methods for Process Identification and Control. Optimization-based algorithms are being developed for the control of constrained, nonlinear processes; these are also being used to control openloop unstable processes. An extended Lyapunov-based stability theory is being developed and demonstrated. In addition, tailored nonlinear programming algorithms are being developed for more efficient solution of these problems.

\section{Carnegie Mellon University \\ Dept of Elec \& Comp Eng Pittsburgh, PA 15213}

$\$ 98,607$ $03-\mathrm{C}$

\section{Research on a Reconfigurable Modular Manipulator System$$
\text { P. Khosla, T. Kanade }
$$

The goal of our research is to address the basic theories of reconfigurable modular manipulator systems that will culminate in the demonstration of these theories on experimental hardware. A modular manipuiator system consists of a set of link and joint modules of various sizes which may be assembled together in a desired kinematic configuration to achieve a specific task. The RMMS design emphasizes mod'slar manipulator components having consistent mechanical and electrical interfaces. The unifom interfaces will allow either semi-skilled field personnel or another manipulator to rapidly configure a RMMS manipulator to meet specific task requirements. This basic research effort will address the problem of mapping tasks into a manipulator configuration, formulation of control algorith:ms for the mapped configuration, and experimental verification of the developed ideas. Though it is not the primary objective, we believe that building prototype experimental modules for demonstrating our ideas will also contribute to the technology of modular manipulators.

For configuring a manipulator from task requirements, we will develop methodologies that map the task requirements into a specific manipulator. The kinematic task requirements will be used to determine the link lengths and the orientation of the modules. And the dynamic task requirements will be translated to obtain the sizes and ratings of the actuators or joints. The use of both rule-based expert systems and optimization techniques will be investigated in obtaining a solution to this problem. For effectively controlling modular manipulators (RMMS), a methodology for automatically configuring a controller will be developed using model-based and/or adaptive control techniques.

\section{University Of Chicago \\ Dept of Chemistry \\ Chicago, IL 60637}

\section{$\$ 0$}

06-C

89-3

\section{Topics in Finite-Time Thermodynamics R. Berry}

The objective of this research is the arıalysis in thermodynamic terms of the perforialance of systems and processes subject to time or rate constraints. Part of the research deals with developing methods for conducting analyses, such as emerged from the introduction of a suitable metric in the space of thermodynamic variables and the evaluation of path lengths with that metric. The lengths so obtained have been shown to be 
directly related to the dissipation associated with the path. Another piece of recent work at this basic level just completed under this project is an attempt to introduce a variational formulation of irreversible heat transfor and diffusion, which is intended to apply in nonlinear as well as linear situations.

The other aspect of this research is the application of the general methods to the analysis of specific systems of current interest. During the initial period of the project, the stopping of a beam of atoms by absorption and re-emission of laser light was analyzed and the entropy changes in the process were evaluated. The reduction in entropy of the atomic beam due to cooling and stopping is compensated a thousandfold over by the increases in the entropy of the light due to randomization of the phase, the polarization and especially the propagation direction.

\section{University Of Chicago}

The Enrico Fermi Institute

Chicago, IL 60637

$\$ 147,420$

$06-\mathrm{C}$

90-3

Fundamentals and Techniques of Nonimaging Optics for Solar Energy Concentration

\section{R. Winston}

Nonimaging optics departs from the methods of traditional optical design to develop instead techniques for maximizing the collecting power of concentrating elements and systems. Designs which exceed the concentration attainable with focusing techniques by factors of four or more and approach the theoretical limit are possible. This is accomplished by applying the concepts of Hamiltonian optics, phase space conservation, thermodynamic arguments, and radiative transfer methods. In the early nonimaging designs the might edifice of aberration theory was dismantled and replaced by a single key idea. According to this, maximum concentration is achieved by ensuring that rays collected at the extreme angle for which the concentrator is designed are redirected, after at most one reflection, to form a caustic on the absorber. This principle proved sufficiently elastic to accommodate most boundary conditions in two dimensions (i.e., linear geometry). Ideal solutions in three dimersions have also been formulated. Our work on vector flux has led to a reexamination of the foundations of radiometry with emphasis on observable effects. Our theoretical work on nonimaging designs has led to demonstration of ultra-high flux from sunlight which exceeds previous results by substantial factors.

\section{Clarkson University Dept of Chemical Engineering Potsdam, NY 13676}

$\$ 59,000$

$01-C$

\section{Lft and Drag Forces on Droplets and Particles in Wall-Bounded Flows J. McLaughlin}

The goal of this research is to obtain quantitative information about the lift and drag forces that act on small droplets or particles that translate through shear flows. Such forces affect the motion of droplets or particles near a rigid wall. Thus, to understand the deposition of droplets or particles on the wall or their tendency to accumulate near the wall, a knowledge of such forces is important.

The approach used in the research involves a combination of analytical, numerical, and laboratory work. The analytical approach can provide useful asymptotic expressions for the lift and drag forces in the regime of small Reynolds numbers. In most situations of practical importance, however, the droplet or particle Reynolds numbers are not small compared to unity. As a result, direct numerical simulations of the three-dimensional flow field around a sphere in a shear flow will provide the forces. In addition, experimental measurements of particle velocities in a vertical homogeneous shear flow apparatus will provide additional information and permit checks of the numerical study.

At present, a computer code has been developed for the direct numerical simulations. The code employs a hybrid spectral-finite volume technique to solve the incompressible Navier-Stokes equation. A homogeneous flow apparatus has been constructed and is being tested at Los Alamos National Laboratory. 
University Of Connecticut

Dept of Mechanical Engineering

Storrs, CT 06268

Micromechanical Viscoplastic Stress-Strain Model with Grain Boundary Sliding E. Jordan

The goal of this research is the development and verification of a model of the time and history dependent viscoplastic deformation behavior of polycrystalline metals. Single grain behavior has been derived by summing postulated slip behavior on crystallographic slip systems. Multi grain behavior will be obtained by summing deformation occurring in different grains while accounting for grain interaction through a self consistent micromechanics approach. The model will also represent grain boundary sliding through the use of a solution recently published by $T$. Mura.

High temperature experiments on Hastelloy $-X$ will be used to verify the model. Single crystal properties used for input to the model are currently being determined. Using these properties the behavior of polycrystal Hastelloy- $X$ will be predicted and then compared with polycrystalline experiments recently completed. Prediction of these tests constitute a critical test for the throry.

The model will not only predict the viscoplastic response of polycrystals but also will allow metallurgical findings usually stated in crystallographic terms to be more directly incorporated into the model. The explicit calculation of grain boundary sliding will help illuminate the role played by grain boundary sliding in the overall deformation during complex load histories. Grain boundary sliding deformation plays an important role in material damage and the model should be useful in life prediction under complex variable temperature conditions.

At present, the single crystal model is available and the self consistent model without grain boundary sliding has been derived and is being programmed.

This work is being done with the cooperation of Engineering Science Software, Inc. working under a related contract. Single crystal specimens have been provided by Pratt and Whitney, Inc.

\section{Cornell University}

Mechanical \& Aerospace Engineering \$59,998 Ithaca, NY 14853

01-A

Chaos in Fluid-Structure Systems

91-3

\section{F. Moon}

Integrated theoretical and experimental studies are being performed to enhance the understanding of nonlinear oscillations and dynamic instability phenomena involving both fluids and solid structures and theil coupling. The objectives are to contribute to the explanation of observed phenomena, providing insights into chaotic characteristics of such coupled mechanical systems and ultimately, to the solution of engineering design problems. This is a joint project with Argonne National Lab.

Fluidelastic instability of loosely supported tubes, vibrating in a tube support plate-inactive mode, is suspected to be one of the main causes of tube failure in some operating steam generators and heat exchangers. As a vehicle to understand the nonlinear behavior of fluid-structure systems, fluidelastic instability of loosely supported tube rows in crossflow is being studied in detail. Tube row with a motion-limiting stop configuration will be tested to investigate various response characteristics and a mathematical model based on the unsteady flow theory will be developed to predict the response characteristics of this classical fluid-structure system.

Energy systems have had a history of dynamic structural instabilities caused by fluid flow resulting in costly component repair and replacement and loss of energy production. The understanding of dynamic characteristics, including periodic oscillations, random vibration, and chaotic motion, will impact the design and reliability of these systems in both the near and long term. in addition, fluid-structure systems are classical examples of autonomous systems that can exhibit chaotic behavior. Knowledge of the fluid-structure systems may provide some insights into the chaotic behavior of other energy system components. 


\section{Cornell University}

Sibley School of Mech \& Aero Eng $\$ \$ 102,000$

Ithaca, NY 14853

\section{Experiments in Turbulent Mlxing z.. Warhaft}

This project is concerned with experimental studies of scalar mixing in fully developed turbulent flows, a subject relevant to our understanding of chemical reactions, combustion and environmental pollution. Mixing in both homogeneous and irhomogeneous turbulence, with and without the effects of buoyancy is being examined.

The first experiment concerns scalar dispersion and mixing in a jet. This is studied using a novel method of ir.troducing a scalar (temperature) into an air jet by means of placing thin heated wire ings, axisymmetrically, downstream from the jet origin. The diameter of the rings and their location from the jet is varied so that the effect of initial conditions on the subsequent evolution of the scalar mixing can be studied. The placing of a thin wire ring in a jet is analogous to placing a line souice in homogeneous turbulence, a subject that has received much attention. We can anticipate that the thermiai field will be more complex than that of a line source since as the flow evolves the thermal field from one side of the flow will begin to mix with that from the other side. This does not occur with a line source in grid turbulence. So far as we can determine there has been no previous study of a heated ring source in a jet. The effects of two scalar mixing is also being studied by adding a second ring. Preliminary findings show unexpected results concerning the evolution of the segregation and cross-correlation coefficients.

The second main experiment is concerned with scalar mixing in stably stratified decaying grid generated turbulence. A temperature (density) gradient is formed at the entrance to the plenum chamber of the wind tunnel and then the flow is passed through a turbulence generating grid. Single and multiple line source dispersion experiments on mixing across sharp temperature steps, with and without buoyancy, are being attempted.
Dartmouth College

Thayer School of Engineering $\$ \$ 140,000$

Hanover, NH 03755

06-C

91-2

Mixing and Settling in Continuous Metal

Production

H. Richter

For reasons of both energy conservation and environmental protection, tonnage exygen has become an essential component in many ferrous and nonferrous smelting and refining operations. Innovative reactors such as the QSL converter for direct lead bullion production are also suitable for direct coppermaking and a modification of the reactor is also very attractive for steelmaking.

Oxygen and fuel are bottom-injected into the molten bath of these essentially horizontal reactors in controlled rates required for heating, physical mixing and chemical reaction purposes. Sufficient turbulence is generated to maximize heat and mass transfer rates. However, such bath turbulence is opposite to the process requirement for continuous countercurrent flow of liquids at specific temperature, compositional and oxygen potential gradients. The powerful stirring effected by submerged gas injection is therefore judiciously localized so as to provide adequately calm settling zones between the active turbulence zones in a staged mixer-settler.

The research objectives of the work are to learn about the behavior of gas and particulate matter in turbulent liquids, the nature and paths of liquids and particulate entrainment into the plumes, and separation phenomena including travel to and behavior in the settling zones. Such knowledge is of fundamental value in designing reactors for sontinuous, direct metalmaking.

The new information will predict bath mixing, heat and mass transfer and settling parameters under the variety of operating conditions which will prevail in continuous, direct metalmaking oxygen reactors of the future. Therefore, it is proposed to study gas injection through submerged injectors, and also particulate addition from above, into a liquid bath consisting of two immiscible layers of liquids having roughly the same viscosities as slag and matte or metal and with roughly comparable density ratios. This work will be initiated in September, 1991. 
Dartmouth College

Thayer School of Englneering

Hanover, NH 03755

$\$ 114,879$

$01-\mathrm{C}$

89-3

\section{Two Phase Potentlal Flow}

G. Wallis

The objective is to develop theorems for twophase potential flow analogous to those existing for single phase flow.

Using one function, the "exertia," which describes the external fluid inertia due to relative motion of suspended particles, it has been possible to derive the average stress tensor, kinetic energy, overall momentum flux tensor and equations of motion foi uniform systems of particles and the effective Bernoulli equation for the fluid flowing through a stationary array of particles.

Recent developments include more general descriptions of the mean particle stresses and the averaged Bernoulli equation for the fluid when both phases have a general motion. Links have also been established between this approach and Guerst's variational methods including the derivation of equations of motion for each phase, it being possible for the dispersed phase to be compressible.

The equations of motion for each phase have also been exnressed in terms of a mutual "interfacial press - -9 tensor" that is related to both the average stress in the dispersed phase and the net force on the continuous phase. This interfacial pressure tensor is also closely coupled to the Reynolds stresses in the continuou. phase.

The exertia and added-mass coefficients have been computed for various geometrical arrangements of spheres, both in an infinite array and in tubes. Comparable measurements were made by measuring the natural frequency of oscillation of such arrays in water. Future experiments are planned in which more properties, such as pressure fluctuations, are measured.

Theoretical and experimental efforts are underway to obtain realistic interphase forces including the effects of viscosity and drag. Computations show that the effects of pressure gradient, added mass phase change and drag do not add linearly except at low Reynolds numbers. This has implications for the transient response and stability of fluidized

\section{Duke Unirersity}

Mech Eng \& Mat'ls Scl Dept $\$ \$ 60,000$

Durham, NC 27706

$01-\mathrm{C}$

90-3

Analytical and Experimental Study of Instabilitles in Buoyancy-Driven Convection in Porous Medla

\section{J. Gecrgladls}

The mission of this research project is the study of thermal instability phenomena associated with natural convection in two classes of fully-saturated porous media systems: (1) porous media saturated with pure fluids, and (2) porous media saturated with binary fluids. Our primary objectives are: (1) the delineation of the various stability domains for the convective flows and an exploration of the convective states after the onset of secondary instabilities, (2) the development of novel experirnental techniques for high precision measurements and determining the flow patterns in porous media, and (3) the validation of macroscopic governing equations for natural convection in porous media.

Emphasis is put on the first two objectives. An experimental investigation of the onset of natural convection in a porous medium with internal heat generation is initiated. The potential of Magnetic Resonance Imaging (MRI) techniques in visualizing low-speed flows in porous media is also investigated. We will explore ways of obtaining quantitative information on interstitial velocity profiles by using a combination of spin tagging and spatial encoding MRI processes. Direct numerical simulation and stability analysis of the pertinent convective states will be performed in conjunction with the experimental component of this investigation.

\section{Engineering Science Software} Smithfield, RI 02917 \$0

A Micromehanical Viscoplastic Stress-A Strain Model with Grain Boundary Sliding K. Walker

The aim of the project is to develop a viscoplastic constitutive model, with accompanying FORTRAN software, to model the deformation behavior of polycrystalline metals comprised of an aggregate of fcc single crystal grains whose crystallographic axes are oriented at random. The single crystal 
grains are assumed to be spherical and are modeled with an anisotropic viscoplastic theory based on crystallographic slip along the octahedral and cube slip directions of the fcc metal. The overall response of the polycrystalline aggregate is assumed to be isotropic and is deduced from the single crystal response by means of a self-consistent method. The effect of grain boundary sliding between the grains is being modeled in the self-consistent formulation to assess the importance of inciuding this mechanism in the overall response of the polycrystalline material.

Experimental tests on single crystal specimens of the superalloy Hastelloy- $X$ are being run at the University of Connecticut to determine the material constants in the single crystal constitutive model. The overall response of the self-consistent model of the polycrystalline aggregate will then be theoretically determined and compared with experimental results from isotropic specimens of polycrystalline Hastelloy- $X$.

The FORTRAN software will allow metallurgical and micromechanical work reported in the literature to be easily embedded in a constitutive (stress-strain) framework for analyzing the overall deformation response of polycrystalline metal aggregates under thermomechanical loading conditions.

This is a joint program with the University of Connecticut under Professor Jordan.

\section{University Of Houston \\ Dept of Mechanical Engineering \\ Houston, TX 77204-4792}
$\$ 36,008$
01-B
91-1

High Flux Film and Transition Boiling L. Witte

This project has focussed on the potential for altering the boiling curve to avoid the unstable transition boiling region through the effects of high subcooling and high velocity. Both experimental and analytical approaches have been taken, with recent efforts concentrating on developing an analytical method by which the influences of high subcooling and velocity on film boiling and pseudo-film boiling in the transition region could be predicted.
Previous efforts have shown that the minimum heat flux can be brought to about $75 \%$ of the maximum flux for highly subcooled Freon-113 flowing over an electrically-heated cylindrical heater. An analysis in which the peak and minimum heat fluxes predicted by correlations of experimental data were equated showed that higher levels of subcooling in Freon-113 might allow the transition region to be avoided altogether with only moderate increases in liquid velocity. Our apparatus is now being re-designed to operate at moderate pressures so that substantially higher levels of subcooling will be achievable. The test section will also be reconfigured so that about a $50 \%$ increase in velocity will also be possible.

An effort to model the film boiling process around a cylindrical heater is well underway. A generalized solution that includes all of the possible effects on the development of a vapor layer around the heater has been achieved up to the point where the vapor layer separates from the heater surface. The influence of variable properties is included and has a substantial effect on how the vapor layer develops around the heater. A technique for matching a wake solution to the boundary-layer-like solution over the front part of the heater has been developed and the resulting equations are now being solved.

The intent of this rart of the research is to yield a method that can be used to study the influence of liquid-solid contact and highly-turbulent frothy flow near the heater surface on the shape of the film boiling curve. If the transition boiling region is to be avoided, then the film boiling prediction must be valid down to heater temperatures typical of the critical heat flux. This analysis will allow the determination of the influence of various system parameters on the shape of the film boiling curve.

\section{Idaho National Engineering Lab Blotechnology and Geosciences $\$ 104,000$ Idaho Falls, ID $83415-2210 \quad 06-A$ 89-3}

Three Phase System Modelling

\section{G. Andrews}

Many unit operations in environmental engineering, including biological wastewater treatment and flotation (used to remove colloids from wastewater and sulfur from coal), involve 
'jubbles rising through $w$ ter containing both surface-active materials 1 id suspended particles. There is no general theory to predict ti:c hydrodynamics near millimeter-sized bubbles in this situation, which makes it difficult to predict the gas/liquid mass transfer rate (in biological wastewater treatment) or the number of particles that strike the bubble (in flotation). This task will develop a mathematical mcdel of the situation on the upper surface of the bubble where most mass transfer and particle capture occurs. The model will be based on integral boundary layer theory for the hydrodyriamics and the transport of surfactant to and from the interface, and will include the effect of adsorbed surfactant on the force balance at the bubble interface.

The results will be compared with experiments designed to measure the particle capture rate as a function of surfactant concentration (i.e., interfacial tension) and particle concentration. Particle capture rate is measured because it is a very sensitive indicator of bubble interface motion.

\section{Idaho National Engineering Lab Applied Optics Idaho Falls, ID 83415 $\$ 290,000$ 06-A 90-3 \\ In-Flight Measurement of the Temperature of Small, High Velocity Particles \\ J. Fincke}

Knowledge of in-flight particle parameters is fundamental io understanding particle/plasma interactions in the physical and/or chemical processing of fine powders. A measurement technique for simultaneously obtaining particle size, velocity and temperature has been developed. Particle size and velocity are obtained from a dual color combination laser Doppler velocimeter (LDV) and laser particle sizing system. The LDV system consists of a crossed beanı technique while particle size is determined from the absolute magnitude of scattered laser light. The particle temperature is determined by a two color pyrometer technique. The spatial resolution is better than $1 \mathrm{~mm}^{3}$ and aliows the distribution of particle size, velocity and iemperature to be mapped over laboratory scale flow fields. The influence of particle size, injection rate, torch power, etc., are currently being examined in typical flow fields.
This project is one of six projects comprising a collaborative research program with the Massachusetts institute of Technology.

\section{Idaho National Engineering Lab Energy \& Systems Tech Group $\$ \mathbf{\$ 2 2 0 , 0 0 0}$ Idaho Falls, ID $83415 \quad 06-A$ 90-3}

\section{Modeling of Thermal Plasma Processes J. Ramshaw, C. Chang}

Optimization of thermal plasma processing techniques requires a better understanding of the space- and time-resolved flow and temperature distributions in the plasma plume and of the interactions between the plasma and injected particles. The present research is directed toward the development of a comprehensive computational model capable of providing such information. The model is embodied in the LAVA computer code for two- or three-dimensional transient or steady state thermal plasma simulations. The plasma is represented as a multicomponent ideal gas governed by the transient compressible Navier-Stokes equations. Multicomponerit diffusion is calculated by a self-consistent effective binary diffusion approximation, including ambipolar diffusion of charged species. Subgrid-scale and k-epsilon iurbulence models are included. Dissociation, ionization, and plasma chemistry are treated by means of general kinetic and equilibrium chemistry routines. Discrete particles interacting with the plasma are represented by a stochastic particle model which allows for distributions in particle sizes, shapes, temperatures, etc. Departures from local thermodynamic equilibrium (LTE) are accounted for by a two-temperature model which permits the electon and heavy-particle temperatures to differ. Nonequilibrium populations of excited levels will be treated as separate chemical species, with collisional and radiative transitions between levels modeled as kinetic chemical rate processes. Applications of the model to date include simulations of plasma jets and plasma spraying. 
Idaho National Engineering Lab Materials Technology Group $\$ 410,000$ Idaho Falls, ID 83415-2218 01-A

Elastic-Plastic Fracture Analysis Emphasis on Surface Flaws

W. Reuter, W. Loyd, J. Epsteln

The objective is to improve design and analytical techniques for predicting the integrity of flawed structural components. The research is primarily experimental, with analytical evaluation guiding the direction of experimental testing. Tests are being conducted on a material (a modified ASTM A-710) exhibiting a range of fracture toughness but essentially constant yield and ultimate tensile strength. As test temperature increases, the specimen configuration-fracture toughness relationship complies initially with requirements for linear elastic-fracture mechanics and extends beyond the range of a J-controlled field. Presently, compact tension and bend specimens are being used to develop state-of-the-art fracture mechanics data for comparisons with data developed from specimens containing surface cracks.

These comparisons are presently underway for 6.4 and $12.7 \mathrm{~mm}$ thick surface-flawed specimens. Metallographic techniques are being used to measure crack tip opening displacement and remaining ligament configurations for comparison with analytical models. Other techniques including .nicrophotography and replicating of the crack tip region to complement the above measurements are being used to identify limits and capabilities of each technique. Moire interferometry techniques are being used to evaluate and quantify the deformation in the crack regior These data are being used to expeiimentally easure $J$ and CTOD for standard (CT and SENB) specimens as well as for specimens containing surface cracks. The above tests have been supplemented by using s,pecimens fabricated from aluminum (dimple rupture only) and titanium. The titanium specimens are being used to study the fracture behavior and the ability of existing models to predict failure for weldments. Moire interferometry techniques are being used to study the local constitutive behavior and the fracture process at the crack tip region of the weldment. Automated techniques are being developed to obtain, store and analyze the moire data.
This project is carried out in collaboration with Professors Parks and McClintock at MIT.

$\begin{array}{lr}\text { Idaho National Engineering Lab } \\ \text { Materials Technology Group } \\ \text { Idaho Falls, ID } 83415 & 03-4,000 \\ & 90-3\end{array}$

Intelligent Control of Thermal Processes H. Smartt, J. Johnson

This project addresses intelligent control of thermal processes as applied to materials processing. Intelligent control is defined as the combined application of process modeling, sensing, artificial intelligence, and control theory to process control. The intent of intelligent control is to produce a good product without relying on post-process inspection and statistical quality control procedures. The gas metal arc welding process is used as a model system; considerable fundamental information on the process has been developed at INEL and MIT during the past six years. Research is being conducted on an extension of the fundamental process physics, application of neural network-like dynamic controllers and signal/image processors, and development of noncontact sensing techniques. Tasks include physics of nonlinear aspects of molten metal droplet formation, transfer, and substrate thermal interaction; understanding the relationship of neural network siructure and associated learning algorithm to mcdel development and learning dynamics in neural networks with the objective of obtaining a fundamental understanding of network transfer functions; and advanced sensing, including the propagation and interaction of ultrasound in metallic solid and liquid media.

This project is part of a collaborative research progiam with the MIT.

\section{Idaho National Engineering Lab Nondestructive Mat'ls Charact Group \$190,000 Idaino Falls, ID 83415-2209 03-B \\ Nondestructive Evaulation of Superconductors K. Telschow}

The purpose of this task is to perform fundamental research which will lead to the development and 
application of new nondestructive evaluation (NDE) techniques and devices for the characterization of high-temperature superconducting materials. In the near future, application of these new superconductors will require NDE methods for evaluating the properties of wires, tapes, and coatings. Microstructural and, particularly, superconducting properties, must be measured noninvasively in a manner capable of providing spatial information so that fabrication processes can be optimized. Although the fabrication of these ceramic materials is being pursued by many different techniques at present, there is enough similarity in the different superconducting materials and the fabricated forms to begin research into NDE measurement techniques. A noncontacting $A C$ induced current measurement technique has been developed which can determine critical currents on a local scale with resolution down to $1 \mathrm{~mm}$. This technique can be used in conjunction with $D C$ transport currents to determine spatial variations in critical current dissipation. It also can be used alone to induce the critical state and full field penetration into the sample directly under the probe. With the aid of the "critical state" model for flux pinning, local critical current values can be determined without any connections to the sample. These measurements are being applied to coating and wire geometries and the results are being correlated with material microstructure information. Nonlinear flux trapping signals are being recorded with a new pulsed probe extension of this technique.

\section{University Of Illinois}

Coordinated Science Laboratory

Urbana, IL 61801

$\$ 122,000$

03-A

91-3

Model Bullding, Control and Optimization of Large Scale Systems

T. Basar, P. Kokotovic

This research program aims at a comprehensive study of some fundamental issues that arise in the modeling, control and coordination of deterministic and stochastic large scale systems. The research plan during the first phase has been to extend the relevant existing theories and methodologies in novel directions, covering areas such as (1) model simplification through decomposition and aggregation, (2) temporal and spatial hierarchies, (3) goal-oriented hierarchies and multiple decision layers, and (4) coritrol and coordination of large scale systems based on (probabilistic) multimodeling.

Some of the specific mathematical models that are being used in this context are Markov chains, stochastic teams, and deterministic and stochastic teams, and dynamic games, each one under a number of different information patterns.

Following this first phase, research is being directed towards developing a new framework, to allow for distributed and interactive model building and control, where both structural and decision making hierarchies evolve over time. A unique characteristic of this approach is that the two independent tasks - modeling and control treated heretofore independently, will now be designed to evolve in parallel and complementarily to each other.

\section{University Of Illinois Dept of Chemical Engineering} Urbana, IL 61801

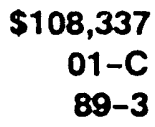

\section{Gas-Liquid Flow In Pipelines T. Hanratty}

Research is being conducted to obtain a better understanding of gas-liquid flow in horizontal and vertical pipelines. The goals are (1) to model slug flow and to predict when slugging will exist in horizontal gas-liquid flows, (2) to obtain improved predictive methods for horizontal and vertical annular flows, and (3) to develop models for the dispersion and deposition of drops and particles in a turbulent flow. Vertical flow experiments are conducted in pipelines with diameters of $3 / 4$ in., 1 $1 / 2$ in., and $21 / 4$ in. The horizontal flow facility has $1,2,3,4$ in. pipelines mounted on a specifically designed platform whose angle can be controlled to within $\mathrm{C} .01$ degree. Droplet motion in turbulent flows is studied in a vertical 2 in. pipeline and in a supercomputer simulation for turbulent flow in a channel. A specifically designed facility that creates stationary slugs in a pipeline is also available.

Considerable progress has been made in developing a new mathematical framework for describing particle motion in horizontal turbulent fields, where gravitational settling can be important. This is being applied to understand 
droplet distribution in gas-liquid annular flows and sediment transport. A Lagrangian framework is used. The central problem, then becomes the description of the behavior of drops or particles originating from a source on the wall. To aid in modelling gas-liquid annular flows, optical techniques are being used to siudy droplet configurations and drop size.

A central problem in understanding the behavior of particles in a turbulent fluid is the prediction of the effect of flow inhomogeneities. This is being studied borh in computer and laboratory experiments. Of particular interest are the discovery of a mechanism by which particles are trapped at the wall and of a relation of particle impaction on a wall to eddy structure.

In previous work necessary conditions for the existence of slugs were developed that viewed the front of a slug as a hydraulic jump and the tail as a Benjamin bubble. These are now being tested in special experiments which create stationary slugs in a downward inclined pipeline.

A comprehensive study of droplet distributions for gas-liquid annular flow in a 4 inch pipeline was completed. These gave the unanticipated result that a secondary flow can be initiated in the gas flow because of the presence of drops.

\section{University Of Illinois At Chicago Energy Resources Center $\$ \mathbf{\$ 7 0 , 0 7 0}$ Chicago, IL $60680 \quad 01-\mathrm{B}$ \\ Momentum and Heat Transfer Processes in $91-2$ Viscoelastic Fluids J. Hartnett}

The goal of the research is to study the fluid mechanical and heat transfer behavior of viscoelastic aqueous polymer solutions. The ultimate objective is to provide a basis for predicting the performance of such fluids. At the present time, two basic investigations are underway:

a) Pool boiling behavior of aqueous solutions of several high molecular weight polymers, including hydroxyethyl cellulose, carboxymethyl cullulose (CMC) and polyacrylamide and a comparison with pool boiling performance of deionized water. b) Forced convection behavior of viscoelastic fluids in laminar flow through rectangular channels.

To date the following results have been found:

a) High concentrations of the aqueous hydroxyethyl cellulose solutions yield higher values of the boiling heat flux than found with deionized water when compared at the same wall-to-fluid temperature difference. In contrast, aqueous polyacrylamide solutions yield lower values of boiling heat flux than found with deionized water when compared at the same wall-to-fluid temperature. A new series of measurements with another cellulose $(\mathrm{CMC})$ is underway to determine if this family of polymers has special boiling properties. Detailed rheological properties are being measured to provide some insight into the boiling behavior.

b) The forced convection heat transfer performance of viscoelastic fluids in laminar flow through rectangular channels is considerably higher than predicted for purely viscous fluids, although the measured friction factor is in good agreement with the value predicted by the purely viscous analysis. Measurements of the dynamic properties including phase shift under oscillatory condition are shown to be related to observed heat transfer behavior.

\section{Jet Propulsion Laboratory Pasadena, CA 91109}

Neural Learning Formallsms for Global 89-3 Manipulator Redundancy Resolution Problems in Unstructured Environments J. Barhen

In many applications the robotic arm needs to have sufficient maneuverability so as to carry out its tasks in the presence of obstacles and in constricted locations. One way to achieve that is to endow the arm with an extra degree of freedom. While mechanically that is easy to implement, say by the addition of an extra joint, the mathematical problem associated with the guidance of the arm becomes very difficult. The reason for that is that with the conventional six-degree-of-freedom system the relationship between the location and 
orientation of the arm's joints and the location of the arm's tip is uniquely determined, while the redundancy accompanying additional degrees of freedom yields an infinity of such relationships. The proposed research will address the resolution of the mathematical problems arising in the latter case.

Specifically, neural networks will be used to permit the robot arm to learn which of the possible motions of the arm are the most appropriate under given conditions. These networks are really models of the way we think, learn, and remember. Those functions are represented by a set of differential equations with many possible solutions, each of which is accessible from well defined initial conditions. In effect the individual solutions serve to encode a learned response to a given stimulus.

\section{Johns Hopkins University Mechanical Engineering Dept Baltimore, MD 21218}

$\$ 69,682$

$01-\mathrm{C}$

89-3

Numerical and Physical Modeling of Bubbly Flow Phenomena

\section{A. Prosperettl}

The ultimate purpose of this study is to put the widely used engineeiing averaged-equations models of multi-phase flows on a firmer basis by comparing their results with those of direct numerical simulations. The first task is the development of suitable numerical techniques for the description of the motion of particles and bubbles in a fluid.

Two different methods have been developed for this purpose. Both the case of potential flow and high-Reynolds-number flow have been addressed. The situations investigated include the small-amplitude oscillatory flow for which the added mass, viscous, and Basset force coefficients have been calculated. The first one is fitted very well by Zuber's cell-theory result, and simple analytical expressions have been derived for the other two. Other problems are the convective transport of spheres in a convergingdiverging nozzle and the buoyant rise of a cloud of spheres.

Currently, the efforts are directed to reducing the computing time needed for the simulations. This requires a careful study of the sensitivity of the methods developed to various possible truncations. In addition, a model to handle the collision of particles - that are found to occur very frequently - is being derived.

\section{Robert H. Kraichnan, Inc.} 303 Potrillo Drive Los Alamos, NM 87544

$\$ 61,694$ 01-C 90-3

Turbulence Theory and Reduced Hydrodynamic Description R. Kralchnan

Turbulent flow is omnipresent in geophysics and in energy-producing devices. Atmospheric turbulent transport plays an essential role in the movement of heat, moisture, and pollutants. Turbulent flows typically contain an enormous amount of information. Both for practicable computation and for physical understanding, it is necessary to extract essential information in compact form. This project explores two recent approaches to economical and meaningful description of turbulence. One is a systematic scheme for reducing the number of degrees of freedom ("decimation"). The other, developed only during the past year, is a method for distorting velocity fields with simple statistics intc self-consistently determined approximations of actual turbulent velocity fields ("mapping closure"). Unlike traditional analytical methods for turbulence, mapping closure yields full probability distributions of velocity fields and contaminants carried by the turbulence. In particular, it can give meaningful estimates of the likelihood of extreme events. Currently, the application of mapping closure to the transport of passive contaminants by three-dimensional random velocity fields is being investigated. Previous work has shown the success of the method for predicting the probability distribution of velocity in Burgers' model of turbulence.

\section{Lawrence Berkeley Laboratory Accelerator \& Fusion Res Div $\$ \mathbf{\$ 1 2 5 , 0 0 0}$ University of California $06-\mathrm{C}$ Berkeley, CA $94720 \quad 90-3$}

\section{Studies in Nonlinear Dynamics}

\section{A. Kaufman, $R$. Littlejohn}

This project involves studies of fundamental properties of nonlinear dynamical systems which 
arise in physical situations of importance to energy research. A major area of theoretical investigation concerns several different aspects of wave systems. Special focus is given to wave packets; the role played by the Maslov index in multidimensional wave problems; the occurrence of Berry's phase in wave and other dynamical systems; the role of periodic orbits in the spectrum of chaotic wave systems; and linear mode conversion. Recent progress includes the exploration of the relation between the Maslov index and the phase space structures of nonlinear Hamiltonian dynamical systems; new methods of computing the Maslov index, both for finding wave functions and eigenvalues; elucidation of the relationship between the Bohr-Sommerfeld and Maslov phases and the recently discovered "Berry's phase"; a discovery of Berry's phase in the adiabatic motion of charged particles in magnetic fields; a study of complex rays in eikonal theory, and their relation to complex Lagrangian manifolds, Stokes' lines and turning points; a numerical exploration of the role of periodic orbits in the spectrum of a chaotic wave system; and the first treatment of mode conversion phenomena for multidimensional systems, involving novel "reduction" techniques. Emphasis is given to the use of a phase space approach to wave phenomena in all these applications. A second area of investigation concerns action principles, which are being used to imbed single-particle Lie transform perturbation methods in collective models, such as Vlasov-Maxwell systems. Nonlinear phenomena (e.g., ponderomotive forces) are thus dealt with systematically.

\section{Lawrence Berkeley Laboratory}

$\begin{array}{lr}\text { Applied Science Division } & \$ 100,000 \\ \text { University of California } & 06-\mathrm{B} \\ \text { Berkeley, CA } 94720 & 91-1\end{array}$

\section{Controlled Combustion}

\section{A. Oppenheim}

The principal objective of this project is to provide a fundamental background for the development of controlled combustion systems. Of particular interest in this respect are essential improvements of combustors for prime movers so that they will provide service not only as power sources, to which they are solely relegated today, but also as modern high-tech chemical reactors, where process instabilities and pollutant formation are adequately controlled by a micro-processor system. For automobile engines this offers the prospect of efficient and clean operation associated with relatively low exhaust temperature, devoid of the problems of knock and cycle-to-cycle variation and meeting practically all air pollutant emission requirements. The major technological constraints imposed upon the automotive industry, the octane number and the catalytic converter, could be therefore eliminated. Means to accomplish this task involve the utilization of a pulsed jet combustion system combined with prompt product recirculation, according to concepts developed on this project. Major laboratory apparatus for our studies consists of a cylindrical vessel with unobstructed optical insight, and a shock tube providing about $20 \mathrm{msec}$ of test time at an elevated pressure (up to 50 atms) and high temperature (around 1000K), commensurate with conditions existing during combustion in engines. Its use is associated with laser-powered optical instruments, including in particular apparatus for megacycle-frequency schlieren cinematography and laser induced fluorescence imagery. In addition to experimental work, included in the program are numerical modeling studies of the fluid mechanic, thermodynamic, and chemico-kinetic phenomena influencing the processes of ignition and combustion in engines.

\section{Lovelace Medical Foundation Bioengineering Research $\$ 50,660$ Albuquerque, NM $87108 \quad 01-\mathrm{C}$ \\ Two-Phase Flow Measurements by NMR E. Fukushima, S. Altobelli, A. Caprihan}

This project will develop nuclear magnetic resonance (NMR) methods to measure velocity and concentration distributions of both phases in two-phase flows. Systems to be studied include concentrated suspension flows in circular tubes, solid-liquid flows in Couette geometries as well as to gas-liquid and liquid-liquid flows.

One approach to velocity measurements is to measure an incremental spatial displacement of nuclear spins that are noninvasively tagged whereas another is to measure the phase evolution of the nuclear spin precession which depends on the translational velocity. The first method is best suited for flows in uniform 
geometries such as pipe flows whereas the second, more complex method, is better suited for non-uniform cases such as for nonneutrally buoyant flows in a Couette geometry.

In order to improve the capabilities of the apparatus to make rapid measurements, a major instrumentation upgrade is being performed during summer and fall of 1991 . This consists in a new operating system and computer, hardware improvements to the if electronics, and a shielded magnetic field gradient coil system. The studies of concentrated suspensions in pipe flow for velocity and concentration distributions is complete except for measurements of particle velocities. The next major experimental emphasis will be on liquidsolid flows in an asymmetric Couette device.

This is a joint project with Sandia National Lab.

\section{University Of Maryland}

Dept of Mechanical Engineering College Park, MD 20742

$\$ 51,999$

03-C

90-3

The Use of Stereo Optical Flow Fields in the Determination of Three-Dimensional Motion and Stereo Correspondence

J. Duncan

In this study, the optical flow fields in the left and right image sequences of a stereo camera pair are being used to determine the three-dimensional motion and position of the camera platform relative to objects in the field of view. A method has been found that uses a robust statistically based calculation to determine the camera motion and feature points in one image that correspond to given features in the other image (stereo correspondence). This method is presently being evaluated experimentally. Preliminary results from tests with synthetic images show that the method produces accurate results when there are not too many points without stereo matches in the two images, when all the points in the left and right images belong to objects with the same threedimensional motion relative to the cameras, when the noise levels are less than about $10 \%$, and when the cameras are accurately mounted in a parallel configuration. Techniques are currently being developed to extend the method to situations where the four conditions mentioned above are not met.
University Of Maryland

Dept of Electrical Engineering

College Park, MD 20742

$\$ 81,745$

06-C

88-4

Mathematical Models of Hysteresis

I. Mayergoyz

This research is concerned with the development of mathematical models of hysteresis. These models are phenomenological in nature and, for this reason, they can be applied to the description of hysteresis regardless of its physical origin.

The main research objectives of the ongoing research can be briefly summarized as follows: development of new generalized Preisach models of scalar hysteresis, study of new Preisach models of vector hysteresis, software implementation of the Preisach hysteresis models, extensive experimental testing and verification of hysteresis models, development of new techniques for the calculation of magnetic fields and eddy currents in media with hysteresis, application of Preisach type models to the description of superconducting hysteresis and evaluation of hysteretic losses in hard superconductors. It is hoped that, as a result of the ongoing research, the foundations of compreherisive theory of mathematical models of hysteresis will be established.

\section{University Of Maryland}

Electrical Engineering Department

Baltimore, MD 21228

$\$ 79,135$

03-B

89-3

Pulse Propagation in Inhomogeneous Optical Fibers

\section{Menyuk}

Our research, which was originally focused on light propagation in inhomogeneous optical fibers, has broadened in scope to include studies of solid state rib waveguides and $Y$-junctions which are used to guide and switch light. The work on optical fibers is divided into two research projects. The first project concerns long-distance communication using solitons. We have been particularly concerned with the effects of randomly varying birefringence, and we have shown that its effect is benign. From the basic equations, we were able to show from an appropriate ordering expansion that the nonlinear Schrodinger equation is the lowest order equation, and, hence, we expect its behavior to dominate the soliton 
evolution even in a highly birefringent fiber, as long as the birefringence is rapidly varying. We have also studied optical fiber soliton switches based on trapping and dragging. To do the work on optical fibers, we have collaborated with scientists at AT\&T Bell Laboratories. The first solid state project was to find the effect of a quantum well on the propagation characteristics of a rib waveguide. Using a planar guide as reference, we were able to show that the effect of the real geometry is qualitatively small but can have a significant quantitative effect. The second solid-state project is to determine the effect of dry-etching on the mode-holding characteristics of the device. As a consequence of the etching, the height at which the junction splits can vary. We showed that the rounding has a very small effect, in contrast to blunting which occurs when the materials are chemically wet etched.

\section{University Of Maryland \\ Dept of Mechanical Engineering \& Systems Research Center College Park, MD 20742}

\section{The Creation of Multi-Degree-of-Freedom Mechanisms for Robotic Applications L. Tsal}

This research is concerned with one of the most difficult stages of mechanical design. The main objective is to develop systematic methodologies for creation of multi-Degree-Of-Freedom (DOF) robotic mechanisms. However, the analysis and control of such mechanisms have also been studied. We discuss progress in the following areas.

1) An innovative concept for controlling gear backlash has been corneived. The concept utilizes redundant unidirectional drives to ensure positive coupling of gear meshes. Then, a systematic methodology for the creation of such mechanisms has been developed. The concept has been proved by an experimental two-DOF manipulator.

2) A systematic methodology has been established for the dynamic analysis of geared robotic mechanisms. It is shown that Lagrange's equations of motion can be derived systematically using the minimum number of generalized coordinates, and that joint reaction forces can be computed on a link-by-link basis. Furthermore, a design methodulogy has been developed for the determination of gear ratios. It is shown that kinematic isotropy and optimal accelerationcapacity can be achieved through proper choice of gearings.

3) Criteria for differentiating transmission characteristics in tendon-driven manipulators have been established. It is shown that the effect of tendon routing can be characterized by a condition number and the direction of the homogeneous solution. In addition, an efficient technique for resolving redundant actuator torques has been developed. The technique offers an opportunity for realtime computed-torque control of such manipulators.

4) In order to gain better understanding of three-DOF, four-jointed wrist mechanisms, trajectories of bevel planetary gear trains are analyzed in detail, workspace and singularity problems are studied. Design criteria for full workspace and maximal four-root region have been established.

\section{University Of Maryland Dept of Mechanical Engineering College Park, MD 20742}
$\$ 127,000$ 01-C 91-3

\section{Lagrangian Analysis of Contaminant}

\section{Dispersal in Bounded Turbulent Shear Flows} J. Wallace, P. Bernard, J. Balint

The objective of this project is to study the physical processes associated with turbulent scalar transport in shear flows with a view towards developing novel techniques for predicting contaminant dispersal in the environment. A closely coordinated experimental and theoretical research program is followed combining wind tunnel experiments using hot-wire probes, smoke visualizations and image processing, with numerical simulations. Concentration contours of point and line source diffusion in the boundary layer obtained from visualization data and from a numerical simulation of channel flow are being compared in order to verify the accuracy of the two techniques. A Lagrangian decomposition of the scalar transport correlation into fundamental physical processes is being carried out using 
ensembles of particle paths computed in the simulation of turbulent channel flow. This provides insight into the physical mechanisms underlying transport and how they may be modeled.

Direct numerical simulation programs including scalar transport have been developed and tested. An algorithm incorporating highly accurate tricubic interpolation for the calculation of particle paths has been successfully implemented and the necessary particle path data is now being computed for evaluation of the Lagrangian scalar transport decomposition. Experimental data from visualization and nine sensor hot-wire vorticity/velocity measurements in a turbulent boundary layer at $R=1000$ are now available. Images were captured on video tape and $16 \mathrm{~mm}$ movie film for subsequent motion analysis and image processing.

\section{University Of Massachusetts} Chemical Engineering Department $\$ \mathbf{\$ 1 6 , 0 0 0}$ Goessmann Laboratory Amherst, MA 01003-0011

\section{A General Procedure for the Synthesis of Process Flowsheets J. Douglas, M. Malone}

This research is focused on developing a more general procedure for the systematic synthesis of flowsheets for chemical processes. These procedures are intended to identify the most important process alternatives, the dominant design variables, and the minimum energy consumption for each alternative in processes containing vapor, liquid-liquid and/or solid phases. This work will substantially expand process synthesis capabilities, building on earlier results which were the basis of a hierarchical synthesis procedure for continuous processes containing mixed vapor/liquid phases.

A hierarchy of decisions and rules for the synthesis of liquid-liquid separation systems and for crystallizer, filter dryer steps have been developed. The generalized flowsheet structure has also been identified and an evaluation of the interactions with other separation steps is being studied. One important difference between vapor/liquid processes and solids processes arises on account of the optimum operating temperature for decanters, dissolvers, and crystallizers. The popular energy integration procedures, which have led to major energy savings in continuous processes over the last decade, must be modified to include this unknown temperature.

Noncontinuous processes which include one or more important batch processing steps are also being studied. An algorithmic approach based on simulated annealing for the scheduling of batch processes including due date and inventory considerations has been developed and the interactions of the design with the production schedule are under study.

\section{University Of Massachusetts- Lowell \\ Department of Electrical Engineering $\$ \$ 88,305$ Lowell, MA 01854 01-C 91-3}

Stability and Heat Transfer in Time-

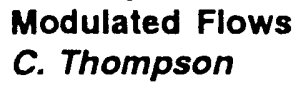

This project represents an analytical/experimental effort directed toward understanding the processes responsible for the generation of boundary layer instabilities in time-modulated flows. In particular the temporal modulations of the basic state by harmonic and free-stream vorticity is considered. The objectives of this work are to: 1) Develop analytical models describing instability in temporally modulated flows. The dynamics of the system is examined using stability analysis and validated against experimental observations. 2) Determine the growth and propagation of unstable disturbances occurring in the viscous region. The influence of the modulation amplitude and wall features such as wall curvature are considered. 3) Examine the stabilizing influence of steady-flow. 4) Examine global and local instability resulting from modulation of the free-stream near points of mean-flow stagnation. 5) Categorize results suggestive of possible mechanisms for heat transfer.

Models for the transition to chaotic fluid motion in time modulation flow have been developed. It has been shown that oscillatory modulation of the basic flow results in successive period-doubling bifurcations of three-dimensional vortical disturbances. Above an amplitude threshold these disturbances exhibit temporally chaotic behavior. 
Massachusetts Institute of Technology

The Energy Laboratory

Cambridge, MA 02139

$\$ 122,300$

03-B

91-3

Metal Transfer In Gas Metal Arc Welding

T. Eagar, J. Lang

The present research is part of a cooperative program among faculty at MIT and staff at the Idaho National Engineering Laboratory (INEL) to develop a sound understanding of the arc welding process and to develop sensing and control methods that can be used to automate the gas-metal arc process.

Previous research on this project mapped the light emissions from a welding arc spatially, temporally, and spectrally. This work identified windows where it is best to photograph or otherwise view the weld pool. Later work developed an understanding of the forces controlling metal transfer in gas-metal arc welding. Welds were made with steel, titanium, and aluminum using a variety of shielding gases and varying the welding current to achieve different metal transfer modes. This work was done using a laser back-lighting technique developed at MIT which allows one to estimate the anode spot attachment on the melding electrode. These experiments have led to a new hypothesis to explain the transition from globular to spray metal transfer. A mathematical model was based on this mode: which accounts for the forces due to gravity, surface tension, electromagnetic, and plasma jet drag. By studying the relative importance of each of these forces, it was shown that 10 to 20 percent higher weld deposition rates could be achieved with pulsed current welding using different shielding gases.

The research during the current year has reviewed methods of filtering the voltage and current waveforms during pulsed current welding in order to extract signals which can be used to control the process. A new process control system has been developed and integrated with the welding equipment. Work has begun to study methods of mechanically controlling droplet detachment from the welding electrode.

\section{Massachusetts Institute Of Technology \\ Energy Laboratory}

Cambridge, MA 02139

$\$ 105,000$

03-A

91-3

Synthesis and Optimization of Integrated Chemical Processes

L Evans

The objective of this research is to investigate and develop improved systematic methods for the synthesis and optimization of chemical processes. The work to date has focused primarily on synthesis of heat and work integration systems for large-scale continuous chemical plants. In the past year we have redirected the project to address methods for synthesis and optimization of batch and semi-continuous processes.

The synthesis of heat and work integration systems in process plant is important in the design of new plants and in the retrofitting of existing plants for energy conversion. A typical process plant, such as a petroleum refinery, a petrochemical plant, or a paper mill, requires energy in the form of heat to raise the temperature of process streams and in the form of work to drive pumps and compressors and meet other mechanical energy needs. The goal of heat and woin integration is to reduce this external energy requirement through better process integration. For example, the heating requirement can be reduced by allowing hot streams that must be cooled to exchange heat with cold streams that must be heated. Also, the process itself can be used to generate mechanical energy by introducing heat engines.

This research has focused on developing computer-based methods to help engineers synthesize a process with improved energy integration. The strategy has been to combine modern mathematical programming techniques, sophisticated rigorous models of the process utilities system and heat recovery network, and heuristic methods to develop an overall methodology that is more powerful than traditional approaches. Methods and computer programming techniques of artificial intelligence have been used where appropriate. An effort was made to overcome the idealizations characteristic of much previous process synthesis research to develop methods that will help engineers solve realistic industrial problems. The net result of the research 
has been an improved understanding of the nature of the problem of energy integration in process plants.

With the completion of three doctoral theses on the project in the area of heat and work integration, the research is now being redirected to address the synthesis and optimization needs of batch and semi-continuous manufacturing processes. These processes are of increasing importance for the manufacture of high-value performance products such as those produced by biotechnology. Yet, the development of systematic computer-based methodologies for batch process is only in its infancy.

\section{Massachusetts Institute Of Technology}

\section{Dept of Mechanical Engineering} Cambridge, MA 02139

$\$ 98,455$ $01-\mathrm{C}$ 89-3

Dryout in Horizontal and Aimost Horizontal Heated Tubes

\section{P. Griffith}

Fluidized bed combustors, waste heat, heat exchangers and combined cycle gas turbine-steam turbine power plants all use horizontal heated tubes with hot gas on one side as the heat source. Because the gas side heat transfer coefficient is so low, excessive heat transfer and burnout is never a problem.

The most important problem is dryout where thermal fatigue or excessive corrosion due to repeated quenching occurs. Dryout can always be eliminatted by increasing the mass velocity in the tube but this increases the operating capense so it is important to know exactly where the dryout limit is.

Pievious work has shown that periodic washing and dryout of the tubes in the intermittent (slug flow) flow regime is the most limiting condition. The primary goal of this project is to determine whether dryout is due to drainage, evaporation or bubble nucleation at the wall. Depending on which mechanism is dominant, a method for predicting dryout in non-uniformly heated, high pressure, large (2") diameter pipes will be developed and tested. The results will be cast in the form of appropriate boundary conditions for a two fluid model of heat transfer in a heated, horizontal pipe.

\section{Massachusetts Institute Of Technology}

$\begin{array}{lr}\text { Lab for Manufacturing \& Product } & \$ 158,000 \\ \text { Cambridge, MA 02139 } & 03-\mathrm{B} \\ & 91-3\end{array}$

Multivarlable Control Of The Gas-Metal Arc Welding Process

\section{Hardt}

The Gas-Metal Arc Welding Process (GMAW) is a highly productive means for joining metals and is being used increasingly for structures and pressure vessels. It presents a challenging multivariable control problem entailing both geometric and thermal outputs. These outputs all combine to determine the strength and toughness of the resulting joint. The overall objective of this work is to examine the problem of simultaneous regulation of all real-time attributes of a weld. Past work has established the viability of independent control of thermal characteristics and the present work is examining the geometric aspects of weld pool control.

One objective of this work is to develop basic process modeling and control schemes to allow independent regulation of the weld bead width and height. A control model relating wirefeed and travel speed to width and height was developed using transfer function identification techniques applied to a series of step welding tests. The resulting discrete transfer function was found to be nonlinear in both the static and dynamic parts owing to a strong dependence upon the input magnitudes. This model was then used to develop a decoupling controller design that cancels the nonlinear behavior using gain scheduling. Proper regulation of the process was demonstrated, but the limited range of control afforded by this process and the significant process delays that were in evidence led to very poor disturbance rejection. It is apparent that the GMAW process will need significant modifications to overcome these problems.

In an attempt to merge previous results on the regulation of thermal characteristics in real-time with the geometry control work, we are developing a control system to independently regulate the weld bead width and the width of the heat affected zone. Initial work is concentrating on simulation of wide seam welding using an analytical heat transfer model as well as a finite difference process model. A key issue in the problem is the 
strong coupling between the inputs (current and travel speed) and the outputs. The use of high frequency transverse motion of the torch is being investigated as a means of overcoming this coupling. Once the control latitude is increased, a two variable control scheme based on both video and infra-red sensing will be implemented.

Finally the depth of penetration of a weld is the most important indicator of weld strength, yet it is the one variable that is essentially impossible to measure directly. A real-time depth estimator has been developed based on solution of an inverse heat transfer problem. Surface temperature measurements are used as inputs to the analytical model, which then tunes the unknown parameters until predicted temperatures match the measured. Initial experiments with this model, using surface temperature measurements from the top and bottom of the weld, have shown accurate and rapid convergence and development of a depth control system based on this estimator is now being processed.

This project is a collaborative program with INEL.

\section{Massachusetts Institute Of Technology \\ Dept of Mechanical Engineering \\ Cambridge, MA 02139}

$\$ 81,000$

$01-D$

91-2

Cryotribology: Development of Cryotribological Theories and Application to Cryogenic Devices

Y. Iwasa

The principal objective of this cryotribology (lowtemperature friction and wear) project is to contribute towards the advancement of cryogenic systems by examining the basic mechanisms of cryotribology. Because relative motion is essential to the operation of many cryogenic devices, an improved understanding of cryotribology will place us in a much better position to meet the future demands that will be required of these lowtemperature system. While continuing to generate more cryotribological data, we have also been expanding our efforts to emphasize the development of cryotribological theories which will enable a more systematic approach towards the design of cryogenic devices, including heliumtemperature $(4.2 \mathrm{~K})$ as well as high-Tc superconducting magnets.

\section{Massachusetts Institute Of Technology} Dept of Mechanlcal Engineering $\$ \$ 196,000$ Cambridge, MA 02139 01-A

Modeling and Analysis of Surface Cracks D. Parks, F. McCllntock

This research focuses on the analysis of ductile crack initiation, growth and instability in part-through surface-cracked plates and shells. The overall approach consists of careful calculations of crack front stress and deformation fields, and correlation of cracking with experimental observations being conducted at the Idaho National Engineering Laboratory.

Recently, significant progress has been achieved in developing and applying a two- $p$ arameter description of crack front fields. The am litude of crack tip deformation is characterized by $J$ or CTOD, while the hydrostatic constraint in the near-tip region correlates well with a parameter related to the so-called T-stress, or second elastic Williams' eigenfunction.

Simplified engineering applications of surface crack analysis are being developed in the context of the line-spring model. Specific enhancements include improved elastic-plastic procedures for the practically important case of shallow surface cracks, as well as simple methods for calculating the T-stress along surface cracks fronts.

Detailed elastic-plastic stress analyses of cracked structural geometries provide a basis for interpreting experimental observations, for quantitatively assessing inherent limitations of nonlinear fracture mechanics methodology, and for extending these boundaries through development of two-parameter characterization of crack tip fields. Simplified but accurate analytical methods are also under development for analysis of surface-cracked plates and shells. Emphasis is placed on better understanding complex three-dimensional features of elastic-plastic crack tip fields. 


\section{Massachusetts Institute Of Technology The Energy Laboratory Cambridge, MA 02139 \\ $\$ 133,000$ \\ $01-A$ \\ 91-3 \\ Basic Engineering Sciences of Sollds Comminution \\ C. Peterson}

This work is part of a broader study of the energy and force requirements for fine grinding of minerals and coal. Research is directed to the crushing behavior of individual particles, down to perhaps 50 microns, and the behavior of particle beds, including in particular the failure of individual particles within such beds.

Experimental work has veen completed on the force and energy necessary to fracture small glass spheres and on the behavior of beds of glass spheres, including the fracture of individual spheres within beds subjected to overall compression. Studies on the fracture behavior of large (one inch) brittle and plastic spheres have been completed.

Analytical work has developed a detailed simulation of the overall and interior behavior of beds of spheres subjected to compression, including the fracture of individual spheres when sufficiently stressed. The simulation was limited to a two-dimensional array of three-dimensional spheres. Correlation of simulated and experimental behavior has been encouraging, but it became clear that an appropriate failure criterion for spherical particles subjected to multiple point loads from neighboring particles was necessary. Four assumed criteria yielded significantly differing bed behavior.

Analytical and experimental work on fracture of individual spheres established an appropriate failure criterion, at least for brittle spheres (which happens to be the simplest of the four assumed criteria). The criterion is also appropriate for plastic spheres subjected to moderate secondary loads, but this is not the case when secondary loads are comparable to the major point load.

With the confirmation of an appropriate particle fracture criterion, at least for most particles, the bed simulation is now deemed suitable for use as a design tool.
Continuing studies have expanded to consider the combined actions of crushing and solids transport with particle beds, with particular attention to the size-dependent relative motion of particles to permit effective removal of fine material as it is formed. A novel comminution device has been conceived to provide the desired crushing and separation, and this concept serves to guide continuing research. Experimental determination of crushing efficiency within beds has shown a maximum efficiency at about $20 \%$ bed compaction, followed by rapidly rapidly declining efficiency at greater compaction as remaining void space approaches zero. Contrary to expectations, thin beds (about 3 narticles deep) are not as efficient as thick beds (greater than 15 particles deep). Preliminary experiments to separate small and large particles with an upward fluid flow have been very successful.

\section{Massachusetts Institute Of Technology}

Dept of Mechanical Engineering $\$ 111,108$ Cambridge, MA 02139 01-C

Rheological and Flow Characteristics of Dense Multiphase Slurrles Employing a Bimodal Model

\section{R. Probstein}

The efficient utilization, transport, and handling of dense multiphase slurries containing high volume fractions of solid particles, distributed in size from submicron to several hundred microns, requires the ability to predict the rheological and flow properties as a function of the various physical parameters including solids loading, particle shape and size distribution, stability characteristics, and solid and fluid properties. Under the grant a rational theoretical and experimental methodology will be developed for the rheological and flow property prediction of dense-phase slurries. The approach models a polydisperse suspension as inherently bimodal, wherein it is considered to be made up of a fine fractior ......h behaves colloidally and imparts to the suspensi, n many of its important rheological and flow characte-istics, and a coarse fraction which behaves as if it were in a pure liquid with the same viscous behavior as the colloidal suspension and raises the apparent viscosity through hydrodynamic interactions. 
The bimodal model has been shown to be very successful in application to a truly bimodal suspension, where the shear dependent viscosity behavior of the colloidal fraction is determined experimentally. For polymodal multiphase flows the bimodal model is being extended, through analysis and experiment, to find a rational method to divide a continuous size distribution into a colloidal and a coarse fraction, and to define the effect of a non-uniform size distribution of each fraction. Additional rheological information on the colloidal fraction, including the effects of the electrochemistry, and the particle shape and size distribution is also being obtained.

\section{Massachusetts Inst Of Tech' Dept of Chemical Engineering Cambridge, MA 02139 $\$ 55,000$ 06-C 91-3 \\ Los Alamos National Lab Design Engineering Division Los Alamos, NM 87545 $\$ 103,000$ 06-C \\ Sandia National Laboratories ${ }^{3}$ 91-3} Fluid \& Thermal Sciences Dept Albuquerque, NM 87185 $\$ 103,000$ 06-C 91-3

\section{Macrostatistical Hydrodynamics}

\section{H. Brenner, A. Graham', L Mondy}

This research aims to correlate the macroscopic rheological behavior of suspensions with their statistical microstructure. This fundamental knowledge will benefit a host of technologies, including geothermal energy production, petroleum production and refining, and synfuels processing. Our approach involves a novel combination of experiments, numerics, and theory. Falling balls settling through transparent suspensions of neutrally buoyant particles are tracked optically; radiography is used for opaque suspensions. High-speed video and image processing serve to observe and record the instantaneous falling ball motion. Nonlinear regression is apnlied to transform between camera and real-space coordinates. Data are interpreted in terms of the average and higher moments of the position and velocity distributions of the falling ball. Experimental measurements covering a large range of conditions show the deviations from the average settling motion of the ball to be much larger in the vertical direction than horizontally. Numerical calculations have been performed to model both a ball falling through a quiescent suspension and the hydrodynamic interaction of many particles in shear flow. The threedimensional dynamics of the interacticns of up to 40 particles have been calculated, and excellent agreement achieved with experimental data.

\section{University Of Minnesota}

Dept of Mechanical Englneering $\$ 0$ Minneapolis, MN 55455

The Impact of Separated Flow on Heat and Mass Transfer

\section{R. Goldsteln}

Separation occurs in many processes involving fluid flow. In the separated flow region reverse and secondary currents (also vortices in some situations) form which give rise to considerable variations in heat or mass tranisfer. Often a small area is covered by the separated flow, rendering a very sharp gradient in the local temperature or concentration. Detection of the occurience of sharp gradients, their extent and the factors influencing them, are the subjects of this study. A mass transfer measurement system capable of sensing minute changes in mass transfer rate over a very small region (measuring data points 0.03 $\mathrm{mm}$ apart) has been developed and is being used. The studies consider flow around the base of circular and square cylinciers projecting out of a plate, flow over roughened surfaces, flow through perforated plates, and flow in the corner and downstream of a bend in a square cross-section duct. Computational modeling of some of these flow cases compares well with the experimental results

\section{University Of Minnesota Dept of Aero Eng \& Mechanics Minneapolis, MN 55455 \\ $\$ 164,268$ $01-\mathrm{C}$ 90-3 \\ Stability Studies of Core-Annular Flows D. Joseph}

The objectives are to understand and control drag reduction in water lubricated pipelines and to determine conditions of stability in water film cooling of water vapor (with phase change) for possible application to nuclear safety. We have developed a successful linear theory for predicting regimes of lubricated flow of oil and water and we 
have new experimental results in lubricated flow in vertical pipes. We are now doing nonlinear theory and preparing experiments on water lubricated pipelining of coal-oil dispersions. We are generally supported and encouraged by Shell Development in Houston. We are world leaders in this subject. We are going to apply the methods we use for water lubricated pipelining to nuclear safety, but we don't know how it will turn out.

\section{University Of Minnesota}

Dept of Mechanical Engineering
Minneapolis, MN 55455

$\$ 134,998$

06-C

88-4

\section{Thermal Plasma Processing of Materials}

\section{E. Pfender}

The obje stive of this research project is to study analytically and experimentally specific vermal plasma processes for materials treatment. Processes of interest include the synthesis of ultrafine ceramic powders and of films.

During the past vear our effort has concentrated on characterizing the thermal plasma chemical vapor deposition (TPCVD) process of diamond films onto various substrates. TPCVD has the advantages that the species thought to be the primary reagents in the deposition of diamond films, namely $\mathrm{CH}_{3}$ and atomic hydroge- are generated in abundance leading to significantly increased deposition rates. The task is to control the recombination processes in the nonequilibrium boundary layer between the plasma and the cooled substrate. In our study we have used the triple torch reactor developed previously under this program to obtain good mixing of the reactants $\mathrm{CH}_{4}$ and hydrogen with the argon - hydrogen plasma jets.

In a systematic study the influences of the pressure in the deposition chamber, of the plasma composition, and of the substrate temperature, material and surface pretreatment have been determined. Polycrystalline films have been deposited on a variety of substrates at surface temperatures between 8,00 and $1200^{\circ} \mathrm{C}$ with deposition rates around $60 \mathrm{~m} / \mathrm{hr}$. Homoepitaxial films have been grown on $1 \mathrm{~mm}$ diameter diamond seed crystals with growth rates up to $200 \mathrm{~m} / \mathrm{hr}$ at temperatures of $1400^{\circ} \mathrm{C}$. The optimum pressure has been found to be around $270 \mathrm{Torr}, \mathrm{CH}_{4}$ to hydrogen ratios of 1 to $6 \%$ have yielded good films. Substrate surface preparation has only affected initial nucleation on super-polished single crystal silicon. Specific film growth mechanisms have been identified for different deposition conditions. Several of these results have not been reported previously.

An initial model has been formulated describing the temperature, velocity and concentration distributions in a plasma reactor approximating the triple torch reactor. This model indicates extremely high temperature and concentration gradients in front of the substrate giving rise to strong thermo-diffusion effects as the controlling mass transport in the boundary layer. Diagnostics will be used to verify the model results.

\section{National Institute Of Standards \& Technology}
Chemica' Eng Science Division
Center for Cliemical Technology
Boulder, CO 80303-3328

\section{Convection and Dispersion in Coarse- Packed Beds M. Jones, J. Wolfe}

One of the most widely used items of industrial equipment is the packed bed in which, commonly, a vertical throughflow exists through a packing which is relatively coarse. The presence of temperature or compositional gradients in conjunction with the stochasticity of the packing on the scale of observation leads to flow structures which may be hard to predict but which are, in any case, not uniform. The flow structures observed under these conditions are being studied with the aid of an array of fiberoptic probes which detect the concentration of a fluorescent dye injected with the throughflow. This technique allows timeresolved imaging of the flow structure in the plane of the array. Considerable insight into the state of convection may be obtained from some of the statistics available from individual probe responses. These include the residence time distribution, the spatial distribution of transit times, and their means and standard deviations.

Experiments have been performed with downward flow of water in a packing of uniform diameter, poured, giass spheres with a vertical temperature gradient. The transition of the flow structure has been observed for a range of temperature 
gradients characterized by a Rayleigh number, and different flow rates characterized by a thermal Peclet number. The transition to mixed forcedfree convection is not abrupt as would be predicted from the volume-averaged equations of motion and energy. Instead, the flow structure undergoes distributions which indicate a random structure which gradually changes to approximate modes determined from the confining geometry. Dispersion obtained from the standard deviation of dye transit times at the plane of observation shows a generally increasing trend, with a small maximum preceding the occurrence of circulating (free convection) flows. The interpretation of these observations is aided by numerical solution of the governing equations for grids with randomly distributed porosity. These show similar precursors to circulating flow associated with neutrally buoyant stagnant regions of the bed.

\section{National Institute Of Standards \& Technology Thermophysics Division $\$ 530,000$ Gaithersburg, MD 20899 03-B Boulder, CO $80303 \quad 91-5$}

Development of Measurement Capabilities for the Thermophysical Properties of EnergyRelated Fluids

R. Kayser, J. Levelt Sengers, M. Moldover, W. Haynes

The major objectives of this project are to develop state-of-the-art experimental apparatus that can be used to measure the thermophysical properties of a wide range of fluids and fluid mixtures important to the energy, chemical, and energyrelated industries and to carry out carefully selected benchmark measurements on key systems. The research is being done jointly by two groups within the Thermophysics Division of the NIST Chemical Science and Technology Laboratory; one group is located in the Gaithersburg, MD laboratories and the other at the Boulder laboratories. The specific measurement capabilities to be developed in this project include new apparatus for transport properties (thermal conductivity and viscosity), for thermodynamic properties (pressure-volume-temperat:ıre data and enthalpy), for phase equilibria properties (vapor-liquid equilibria, coexisting densities, and dilute solutions), and for dielectric properties (dielectric constant). These new apparatus will extend significantly the existing state of the art for properties measurements and make it possible to study a wide range of complex fluid systems (e.g., highly polar, electrically conducting, and reactive fluids) under conditions which have been previously inaccessible. This project also includes benchmark experimental measurements on systems containing alternative refrigerants, on aqueous solutions, and on carefully selected systems with species of diverse size and polarity that are important to the development of predictive models for energy-related fluids.

\section{National Institute Of Standards \& Technology \\ superconductor \& Magnetic \\ Measurements Group \\ Electromagnetic Technology Div \\ $\$ 98,488$ \\ Boulder, CO 80303 \\ $06-\mathrm{C}$ \\ 89-3}

\section{Low Resistivity Chmic Contacts Between \\ Semiconductors arid High-T \\ Superconductors \\ J. Moreland, J. Ekin}

The purpose of this project is to fabricate and characterize contacts between high-T superconductors (HTS) and semiconductors. Developing a method for optimizing the current capacity of such contacts will extend the application of HTS materials to semiconductor hybrid technologies that have HTS interconnects (both on-chip and package) and proximity HTS/ semiconductor/HTS SNS Josephson Junctions. Presently, these are some of the most promising HTS applications, but an essential first step is the development of stable ohmic contacts between semiconductors and HTS materials.

The project is now focused on $\mathrm{YB}_{2} \mathrm{Cu}_{3} \mathrm{O}_{x}(\mathrm{YBCO})$ thin-films grown on Si wafers. Recent discoveries have shown that is possible to grow high quality HTS films on Si using laser ablation deposition and a yttria stabilized zirconia buffer layer between the YBCO film and the Si. Processing steps are being optimized for depositing and patterning YBCO films on $\mathrm{Si}$ to form contact vias to the $\mathrm{Si}$ substrate. The goal is to minimize the specific resistance of the contacts using techniques that are compatible with standard Si processing. 


\section{The City University Of New York}

The City College

The Benjamin Levich Institute

\$o

New York, NY 10031

\section{The Rheology of Concentrated Suspensions \\ A. Acrivos}

This research program aims to investigate the flow of concentrated suspensions of non-colloidal particles from the fundamental point of view. Earlier studies by the principal investigator and his associates have shown that the rheology of such systems is strongly affected by the shear-induced migration of particles from regions of high shear to low and from high concentrations to low which, by distorting the particle concentration profile, can lead to an erroneous interpretation of the experimental measurements. This shear-induced particle diffusion is also responsible for the phenomenon of viscous resuspension whereby, in the presence of a shear flow, a settled bed of heavy particles can resuspend even under conditions of vanishingly small Reynolds numbers.

Viscous resuspension is being studied both theoretically and experimentally in various unidirectional flows, e.g. a 2-D Hagen-Poiseuille flow, as well as a Taylor-Couette system. Excellent agreement has been found to-date between the experimental results and the theoretical predictions even though the latter do not entail the use of adjustable parameters.

\section{The City University Of New York The City College $\$ 120,000$ The Benjamin Levich Institute 03-A of Physico-Chemical Hydrodynamics 91-3 New York, NY 10031}

\section{Partial Control of Complex Processing Systems \\ R. Shinnar, I. Rinard}

In many instances the control of a process can be managed by accessing and manipulating only a limited number of variables in the presence of many. The inevitable problem is how to exercise the necessary choices. While empirical, practical rules for making those choices exist, there is as yet no sound theoretical basis for solving problems in partial control of complex processes.
In general the number of variables to be controlled must be equal to the number of variables available for manipulation. However experience shows that often satisfactory results can be obtained by controlling a smaller number of variables. That is referred to as practical controllability. The object of the proposed research is to provide a rational basis for making optimal choices of the necessary variables.

To focus the research, it is proposed to address this basic problem in the context of a Fluidized Bed Catalytic Cracker (FCC). In particular the question of the dependence of controllability on the system design will be addressed. The choice of this example is governed by the availability of a reasonable nonlinear model of FCC. The specific steps to be taken will include a determination of operating set points for different measured variables and different control variables, the dependence of process stability on the choice of those variables, and implementation of a particular type of partial control, viz., cascaded control.

In addition to the FCC, it is proposed to carry out a similar investigation of partial control of polymerization reactors.

\section{The City University Of New York The City College $\$ \$ 98,500$ The Benjamin Levich Institute 06-C of Physico-Chemical Hydrodynamics 91-3 New York, NY 10031}

\section{Studies in Premixed Combustion G. Sivashinsky}

The objective of this research is a combined analytical and numerical study of the influence of various hydrodynamic, thermal-diffusive and reaction rate factors on speed, shape, stability and the extinction limits of premixed gas flames. Differerit modelling techniques will be employed to reduce the study of pertinent combustion systems to simple approximate problems tractable either analytically or numerically. Specifically, the project is concerned with (1) the dynamics of premixed flames spreading through a one- and multiplescale system of eddies and with the further development and refinement of the associated cascade-renormalization concept of turbulent flame speed; (2) the intrinsic dynamics and the pattern formation in premixed flames sustained by 
the thermal-diffusive and thermal expansion induced mechanisms of flame instability; (3) the hydrodynamical aspects of combustion limits with particular attention to the phenomena of flame extinction by one- and multiple-scale periodic flowfields and extinction in upward and downward propagating flames in tubes; and (4) the development of new numerical methods especially assigned for the free-boundary problems of flame-flow interaction and spontaneous pattern formation in premixed flames.

\section{North Carolina State University Dept of Mech \& Aero Eng \$95,533 Raleigh, NC 27695-7910 06-A 89-4 \\ Transport Properties Of Disordered Porous Media From The Microstructure \\ S. Torquato}

This research program is concerned with the quantitative relationship between transport properties of a disordered heterogeneous medium that arise in various energy-related problems (e.g. . thermal or electrical conductivity, trapping rate, and the fluid permeability) and its microstructure. In particular, we shall focus our attention on studying the effect of: porosity, spatial distribution of the phase elements, interfacial surface statistics, anisotropy, and size distribution of the phase elements, on the effective properties of models of both unconsolidated media (e.g., soils and packed beds of discrete particles) and consolidated media (e.g., sandstones and sintered materials).

Both theoretical and computer-simulation techniques have been employed to quantitatively characterize the microstructure and compute the transport properties of disordered media. Statistical-mechanical theory has been used to obtain $n$-point distribution functions and to study percolation phenomena in continuum random-media models. This has led to accurate predictions of transport properties of realistic models of isotropic as well as anisotropic heterogeneous media. An efficient Brownian motion computer-simulation methodology has been developed to exactly yield effective diffusion properties such as the conductivity, dielectric constant, magnetic permeability, diffusion coefficient, and the trapping rate associated with diffusion and reaction among sinks. The first rigorous relation which links the fluid permeability to the electrical conductivity of porous media has been derived. Work has begun on the study of the dispersion of a passive tracer due to random velocity fields.

\section{Northwestern University Dept of Civil Engineering Evanston, IL 60208}

Quantitative Non-Destructive Evaluation of High Temperature Superconducting Materials J. Achenbach

A crack in a solid body can, in principle, be detected and characterized by its effect on an incident pulse of ultrasonic wave motion.

The work on this project is concerned with applications of the scattered field approach to the detection and characterization of cracklike flaws. The work is both analytical and numerical in nature. Several forward solutions to model problems have proven to be very helpful in the design of experimental configurations. They are also valuable in interpreting scattering data for the inverse problem.

The efficacy of ultrasonic methods to detect and characterize a crack depends on topographical features of the crack faces, the presence of inhomogeneities in the crack's environment, and on the mechanical properties in the near-crack region. In this work the effects on the scattered ultrasonic field of various features of fatigue and stress corrosion cracks, such as partial crack closure, the presence of microcracks and microvoids, and near-tip zones of different mechanical properties have been investigated. Most of the results have been obtained by formulating a set of singular integral equations for the fields on the boundaries of the scattering obstacles. These equations have been solved numerically by the boundary element method, and the scattered fields have subsequently been obtained by using representation integrals.

For the configurations examined in this work, crack closure has the most significant effect on far-field scattering. 
Northwestern University Dept of Chemical Engineering Evanston, IL 60208

Thinning and Rupture of Thin Liquid Films S. Bankoff, S. Davis

The objective of this work continues to be the study of the dynamics, stability, and rupture (including contact-line motions) of thin liquid films, especially with heat and/or mass transfer. The two- and three-dimensional structure of surface waves on a heated inclined plane, has been examined and the experimental technique has been developed to measure accurately the surface-wave shapes, frequencies and amplitudes on thin liquid films draining down an inclined plate under adiabatic or heating conditions, for comparison with theoretical predictions.

The evolution equation for two-dimensional disturbances incorporates viscosity, gravity, surface tension, thermocapillarity, and evaporation effects. The linear theory derived from this describes the competition among the instabilities. Numerical solutions of the evolution equation describe the finite-amplitude behavior that determines the propensity for dryout of the film. Among the phenomena that appear are the tendency to wave breaking, the creation of secondary structures, and the pre-emption of dryout by the mean flow. A three-dimensional evolution equation was also derived that contains surface-wave instability and nonlinear saturation. A new secondary three-dimensional instability was identified. Unlike other secondary instabilities found in many shear flows, the instability does not require a threshold amplitude, so that all twodimensional permanent waves on vertical plates become unstable to three-dimensional disturbances. The nonlinear evolution of threedimensional layers, including the finite-time behavior of the secondary instability, is studied by posing various initial-value problems and numerically integrating the long-wave evolution equation.

\section{Northwestern University}

Dept of Eng Scl \& App Math

Evanston, IL 60208

$\$ 51,000$

$01-\mathrm{C}$

91-3

Effects of Capillarity of Macroscopic Flow in

Porous Medla

M. Miksis

The objective of this project is to study the effects of capillarity on the motion of a fluid in a porous material. The primary concern will be the micromechanics of the fluid motion when interfaces are present and its effect on the macroscopic flow. These interfaces could exist as a liquid moves into an unsaturated porous material or when there are two phases within the porous material, e.g., liquid/gas or liquid/liquid. Attention will be directed to understanding and modeling the dynamics of these interfaces and then relating their dynamics to the macroscopic behavior of the material.

One of the central concerns of this work will be to understand how the motion of the contact line, i.e., the line of intersection of a liquid/liquid or liquid/gas interface and a solid, influences the motion of the fluids. Recent work has been directed to solving for the evolution of a contact line using analytical and numerical methods. Also recently the dissipation associated with the motion of a contact line and how this dissipation manifests itself in a macroscopic model of wave propagation in a partially saturated porous material has been investigated. The microscopic flow problems in a porous material can now be studied using these results. Also attention will be directed to problems concerned with the fundamental modeling of the motion of a contact line. This is an important aspect of the work since the dynamics and dissipation associated with the motion is very dependent upon the model used for the contact line motion. 
Northwestern University

School of Eng \& Applied Sclence

Evanston, IL 60208-3120

$\$ 210,155$

$06-C$

91-3

Mixing of Immiscible Fiuids in Chaotic Flows and Related Issues

\section{J. Ottino}

In spite of its universality and practical implications, the understanding of the fundamentals of mixing remains rather incomplete. This work focuses on basic experimental and computational studies along two main themes with the objective of establishing a framework capable of addressing mixing problems encountered in nature and technology: (1) investigations of chaotic mixing of single fluids in deterministic twoand three-dimensional flows, and (2) dynamics of microstructures in such flows (e.g., stretching, breakup, coagulation, etc.). Work in area (1) is underway and a clear picture is emerging. Experimental work is planned to address the problems listed under area (2). However, most of the results to-date are due to simulation which focused on coagulation of point particles in chaotic flows. The particles are convected without diffusion and allowed to coagulate with probability one when their mutual distance is less than their diameters. The most significant finding is that under "well mixed" conditions the system behaves as if the particles were moved by Brownian motion and a simple kinetic model describes the main results. The poorly mixed case is considerably more complex. in this case spatial inhomogeneities result from competition between the rate of coagulation and mixing, and trapping and leaking of clusters due to KAM surfaces.

\section{University Of Notre Dame}

Department of Chemical Engineering $\$ 52,000$ Notre Dame, IN $46556 \quad 01-C$

Study of Interfacial Behavior in Cocurrent 91-3

Gas-Liquid Flows

M. McCready

The objectives of this work are to develop an understanding of various types of small-scale (e.g. waves) and large scale (e.g. slugs) disturbances which are observed in cocurrent gas-liquid flows.

Typical gas-liquid systems are "open" flows where there is no feedback of information between the inlet and exit. It is important to determine to what extent the ubiquitous time-varying fluctuations at the inlet, which may be of small or large amplitude, can be expected to affect the qualitative or quantitative behavior of the downstream flow. For example, it appears that close to neutral stability in a stratified flow, conditions exist where the irregularity in the interfacial waves can be traced to inlet noise. However for other nearby conditions the waves show no evidence of the effects of inlet noise.

The present approach is to characterize these flows by using experimental measurements of wavelength and amplitude for periodic waves, and amplitude and time-spacing between irregular disturbances. These measurements are interpreted using linear and nonlinear analysis, based on spatially-varying disturbances, of the Navier-Stokes equations. Additional insight comes from some of the recent developments in understanding the generic behavior of nonlinear dynamical systems (e.g. intermittency and chaos) but with careful recognition of the differences between open systems and the typically-studied closed systems (e.g. Taylor-Couette flow).

\section{Oak Ridge National Laboratory Engineering Physics $\$ 972,000$ and Mathematics Division 03-C Oak Ridge, TN 37831 \\ $87-5$}

\author{
Center for Engineering Systems Advanced \\ Research (CESAR) \\ R. Mann, F. Pin
}

The Center for Engineering Systems Advanced Research [CESAR] is established at Oak Ridge National Laboratory to address long-range, energy-related research in intelligent machine systems. These systems are intended to plan and perform a variety of tasks in incompletely known environments, given only qualitatively specified goals. The Center provides a focal point for interdisciplinary research in machine intelligence, cognitive systems, advanced control, and systems engineering. Research objectives are chosen to address the technology-base requirements for DOE missions that rely on the use of robotics and intelligent machines. In particular, research concentrates on issues related to autonomous systems, unstructured dynamic work environments, and multiple cooperating robotic 
systems. Results from CESAR research in automation-related technologies and intelligent machines can increase productivity and safety in the development and operations of DOEsponsored systems. Potential and actual applications include emergency situations, remote operations, resource exploration, transportation systems, advanced power generation systems, and environmental restoration and waste management.

CESAR is intended to be a national resource, and a major objective is to disseminate $R \& D$ accomplishments freely and comprehensively. Results and technology advancements are distributed through publications in the scientific literature, through organization of workshops on selected topics, and through the development of prototype systems that demonstrate concepts and new methodologies. CESAR cooperates with universities, laboratories and industry, serving as a collaborative research facility to provide guests with access to state-of-the-art and often unique equipment in a stimulating research environment.

\section{Oak Ridge National Laboratory Chemical Technology Division $\$ \$ 103,000$ Oak Ridge, TN $37831 \quad$ 06-A \\ 89-3 \\ Bioprocess Engineering Research for Energy Applications \\ C. Scott}

This program will support engineering research in three primary areas that are critical to an expanding use of advanced bioprocessing systems for energy production and conservation: efficient biocatalytic systems, (2) innovative bioseparations approaches, (3) advanced bioreactor concepts.

Efficient biocatalytic systems - Several advanced bioreactor concepts require that the biological catalyst (microorganisms or active fractions) be retained in the bioreactor at high concentrations in a microenvironment that enhances biological activity. A fundamental understanding of the basic kinetics and dynamics of such biocatalyst systems and a predictive mathematical model will be developed.

Innovative bioseparations approaches - Advanced separations concepts will be needed to isolate or concentrate the dilute aqueous components of bioconversion systems or waste streams from energy processes. The research emphasis will be on separation concepts that utilize solid-fluid interactions with an initial study of the use of biological materials (certain microorganisms and plant and animal tissue) as bioadsorbents for the isolation and concentration of dissolved metals.

Advanced bioreactor/biosorber concepts - Fixedand fluidized-bed bioreactors and/or biosorbers using immobilized bioreagents are advanced concepts that have the potential for significantly increasing productivity. However, such systems are not well defined, since they may contain three or four interacting phases, including a changing gas phase. The coupling of hydrodynamics, gas generation or depletion, gradients of phase fractions, and biokinetics in continuous columnar systems will be investigated and predictive mathematical models will be developed.

\section{Ohio State University}

Physics Department

Columbus, $\mathrm{OH} 43210$

$\$ 66,000$ $06-\mathrm{C}$

91-3

Turbulence and Spatio-Temporal Chaos

C. Jayaprakash, F. Hayot

This program deals with the dynamics of nonlinear systems. It concerns three significant areas of research:

(1) the study of spatial coherence and temporal chaos in systems with many degrees of freedom: as model systems, coupled maps on d-dimensional lattices will be investigated.

(2) the application of renormalization group techniques to non-equilibrium systems, and the problem of understanding non-linear partial differential equations by deriving effective stochastic ones at long wavelengths.

(3) a systematic study of amplitude equations for various physical situations, with emphasis on the effects on patterns of non-variational terms in the equations. 


\section{Oregon State University Dept of Mechanical Englneering Corvallis, OR 97331-6001 \\ Radiative Transfer Through an Array of Discrete Surfaces J. Welty}

Radiation heat transfer in arrays of discrete surfaces is an important class of radiative transfer problems. Analytical solutions are difficult for even the simplest array and, as an array increases in complexity, the computational time for a traditional Monte-Carlo model becomes excessive, even for supercomputers.

This experimental study is designed to explore basic relationships between array geometry (spacing, packing arrangement, and element shape), surface properties, and radiative transfer through an array. The information resulting from this study may also be useful in establishing criteria for the valid application of participating media models to arrays of fixed discrete surfaces.

The experimental apparatus is in the development and initial testing stage. It consists of a uniform, high intensity, collimated, chopped, visible light source; a two-dimensional axi-symmetric array; a highly directional fiber-optic probe connected to a PMT; and a lock-in amplifier. Initial experiments will involve two element shapes, one with a circular cross-section and one with a high-aspect-ratio rectangular cross-section; and two types of surfaces, highly reflective specular and highly reflective diffuse. An important use of the initial results is verification of a numerical code that will be used to direct subsequent experimental investigations.

This work is being carried out in cooperation with Battelle, Pacific Northwest Laboratory.

\section{Pacific Northwest Laboratory Battelle Memorial Institute $\$ \mathbf{\$ 7 5 , 0 0 0}$ Richland, WA 99352 01-B \\ Radiative Transfer Through Arrays of Discrete Surfaces with Fixed Orientation M. Drost}

Radiation heat transfer in an array of discrete surfaces is an important and poorly understood class of radiative heat transfer problems. The objective of this study is to develop an understanding of the impact of array geometry, surface properties, and incident radiation characteristics on radiation heat transfer in the array. The results of the study will be used to establish criteria for the valid application of participating media models to arrays of fixed discrete surfaces.

The approach consists of using an innovative Monte Carlo model optimized for evaluating arrays of fixed discrete surfaces to evaluate radiation heat transfer in arrays with a range of geometric characteristics. The Monte Carlo model will be validated by comparison with experimental results being developed by Oregon State University in a parallel study. Following validation, the Monte Carlo model will be used to conduct parametric evaluations of a range of array geometries. The results of the parametric studies will be used as a benchmark for comparison with analytical approaches that model the array as a participating medium.

FY 1991 accomplishments consist of initiating Monte Carlo code modification to allow comparisons with experimental results and simulations used to assist the design of the experimental apparatus.

\section{University Of Pennsylvania}

Electrical Engineering Department \$108,502 Philadelphia, PA 19141

$06-C$

91-1

Radiative Interactions with Micromachined

Surfaces: Spectral Angular Polarized

Emittance

\section{J. Zemel}

Recent theoretical and experimental studies have shown that the basic approach leading to the conventional views about the nature of black body radiation fails when the size of the cavity is comparable with the wavelength of the incident radiation. Such a situation can arise when light interacts with periodic microgrooves inscribed on a surface. The detailed nature of such an interaction may be studied by means of spectral, angular, polarized emittance (SAPE).

Preliminary results show that SAPE allows the measurement of the effects of thermally excited standing waves in the microgrooves on the 
scattering of the incident radiation. It was found that indeed there are standing waves in the interior of the microgrooves, or slots. Those waves couple efficiently to the incident radiation as well as to the surface plasmons. The measured dependence of the latter coupling on the depth of the slot cannot be accounted for fully by the existing theory. Measurements of the radiation scattered by the microgrooves shows that spontaneous emission is inhibited in some directions. It is possible that this unusual behavior may find eventually practical applications in electro-optic devices.

This research program call for determining how the geometry of the microgrooves affects the interaction between the incident radiation and the standing waves inside the slots; determining the role played by the material properties of the micromachined surfaces on the polarized, angular emission; and determining the necessary corrections to the Stefan-Boltzman radiation law necessitated by the small size of the scattering cavities.

\section{Physical Optics Corporation} $\begin{array}{lr}2545 \text { W. 237th Street, Suite B } & \$ 154,621 \\ \text { Torrance, CA } 90505 & 06-B \\ 89-3\end{array}$

Nonuniform Ljouville Transformers for Quasi-Homogeneous Optical Fields J. Jannson

In this program, Nonuniform Liouville Transformers (NLT) are proposed for an important class of quasi-homogeneous optical fields containing those emitted by thermal (Lambertian) and other convectional sources, as well as by the majority of multi-mode high-energy $\left(\mathrm{CO}_{2}, \mathrm{Nd}\right.$ :YAG, Excimer, $X$-ray, etc.) lasers and semiconductor lasers (including laser diodes (LD), which are important sources in local area networks (LANs)). These NLTs are nonuniform, nonimaging beam-shaping systems acting on the basis of the general energy transport equations for the phase-space density (related to the generalized Liouville theorem) and the power flux vector. The proposed theoretical model is more general than those based on geometrical optics (including Winston's nonimaging optics), yet less general than that based on either the scalar diffraction or electromagnetic theory. The fundamental goal of this program is to find a theoretical and engineering solution for highly efficient (close to $100 \%$ ) beam shaping/concentration/ collimation of weakly spatially coherent and quasi-uniform large-aperture optical fields (so called quasi-homogeneous fields, which are more general than Lambertian ones), using an optimized higher-hierarchy architecture of longitudinal and transversal, imaging and nonimaging optical elements (nonimaging cones, lenses, diffusers, holographic optical elements (HOEs)). The proposed theoretical model holds for the quasi-homogeneous radiation emitted by the state-of-the-art of the majority of high energy and coherent and noncoherent electromagnetic sources.

\section{Physical Sciences Inc.} 20 New England Business Center Andover, MA 01810

$\$ 123,110$ 06-A 88-4

\section{Experimental and Theoretical Studies of Condensation in Multicomponent Systems M. Frish, G. Wilemski}

This research program comprises experimental and theoretical studies of nucleation and condensation in multicomponent gas mixtures. The program goals are: (1) to improve basic understanding of nucleation and droplet growth, (2) to stringently test theories of nucleation at high nucleation rates and under nonisothermal conditions, (3) to develop improved theories where needed, (4) to enlarge the data base for systems of both fundamental and practical interest, and (5) to provide reliable means for predicting the behavior of mixtures used in practical applications such as turbo-machinery. Experimentally, condensible vapors mixed with a carrier gas are cooled in a supersonic nozzle to obtain high nucleation rates. The nozzle is designed to ensure that steady-state nucleation occurs and to give satisfactory spatial resolution of the temperature profile. Laser light scattering and interferometry are used to detect the "onset" of condensation and to monitor subsequent droplet growth.

Experiments on condensing water vapor have shown that the number density of water droplets falls sharply after an initial rapid increase. This previously unobserved behavior appears to be due to polydispersity of the droplet size distribution which favors growth of larger droplets at the 
expense of smaller ones. Theoretical calculations of the droplet size distribution along the flow axis support this interpretation. The calculated size distribution broadens rapidly; large numbers of large droplets (9 $\mathrm{nm}$ radius) are already present at the observable onset of condensation. The rapid growth in number and size of these large droplets is responsible for the latent heat release that disturbs the gas flow. These results contradict the conventional view of condensation in nozzles. In that view, onset is attributed to the rapid nucleation of a monodisperse cloud of very tiny microclusters $(0.4 \mathrm{~nm}$ radius) which then grow in a number-conserving fashion. This work shows that nucleation and condensational growth do not occur in separate stages beginning at onset, and it establishes both the presence and importance of large droplets at the onset of condensation.

\section{Princeton University Dept of Mech \& Aero Eng Princeton, NJ 08544}

$\$ 91,859$ 06-B 90-3

Mechanisms and Enhancements of Flame Stabilization C. Law

This program aims to gain fundamental understanding of the structure and stabilization of premixed and diffusion flames through theoretical and experimental investigations. Two projects were completed during the reporting period.

In the first project, it is demonstrated that conventional activation energy asymptotic analysis of premixed flames lacks an additional relation for complete closure except for some special cases, that this deficiency is caused by the approximation of the reaction term as a delta function in the reaction-sheet limit, and that imposition of arbitrary closure relations could lead to $O(1)$ errors in the solution. It is subsequently shown that closure, to the needed $O(E)$ accuracy in the solution, can be achieved by locating the reaction at the "center of reaction", which is based on the first moment of the reaction rate function about the location of its maximum point.

In the second project the structure and stabilization mechanism of an inverted flame situated over a thin rod in a uniform flow has been studied both experimentally and theoretically. The results seem to indicate that stabilization can be achieved without heat loss to the rod, and that flame curvature has a significant effect on the flame response.

\section{Purdue University}

School of Mechanical Engineering

West Lafayette, IN 47907

$\$ 88,901$

$01-C$

90-3

Effect of Forced and Natural Convection on

Solidification of Binary Mixtures

\section{F. Incropera}

This study deals with the influence of combined convection mechanisms on the solidification of binary systems. A major accomplishment of research performed to date has been the development and numerical solution of a continuum model, which uses a single set of equations to predict transport phenomena in the liquid, "mushy" (two-phase), and solid regions of the mixture. Calculations have been performed for two-dimensional, aqueous salt solutions involving forced convection, thermo/solutal natural convection, and/or thermo/diffusocapillary convection. The calculations have revealed a wide variety of rich and robust flow conditions, including important physical features of the solidification process which have been observed experimentally but have heretofore eluded prediction. These features include doublediffusive layering in the melt, development of an irregular liquidus front, remelting of solid, development of flow channels in the mushy region, and the establishment of characteristic macrosegregation patterns (regions of significantly different composition) in the final solid.

The primary objective of current studies is to determine the manner in which externally imposed forces influence thermo/solutal convection in the mushy and liquid regions during solidification of a binary mixture. A special goal is to determine means by which the forces may be used to offset or dampen thermo/solutal convection, thereby reducing macrosegregation and attendant casting defects. Separate consideration is being given to the effects of magnetic and centrifugal forces on solidification in binary metallic alloys and aqueous salt solutions, respectively. Predictions based on the continuum model are being compared with measurements obtained for meta!lic $(\mathrm{Pb}-\mathrm{Sn})$ and aqueous $\left(\mathrm{NH}_{4} \mathrm{Cl}-\mathrm{H}_{2} \mathrm{O}\right)$ systems. 
Purdue University

School of Nuclear Engineering

West Lafayette, IN 47907

$\$ 104,000$

$01-\mathrm{C}$

90-3

Interfacial Area and Interfacial Transfer in

Two-Phase Flow

M. Ishil

The objective of the research program is to develop instrumentation methods, experimental data base and analysis leading to predictive models for describing the interfacial structure and behaviors of two-phase flows. In terms of the flow structure, the local void fraction, interfacial area concentration, fluid particle size distribution and flow patterns in vertical two-phase flow systems are studied in detail. For the purpose of understanding the dynamic behaviors, the interfacial velocity, wave characteristics and coalescence and disintegration of fluid particles are investigated. Special emphasis is placed on developing the four-sensor and five-sensor resistivity probe method and improving the double-sensor resistivity probe method. A second emphasis is on the system size and entrance effects on the interfacial characteristics described above. The changes in the interfacial structures and flow regimes as a function of an axial location are measured experimentally. This information together with the local measurements from the probe method are used to study the mechanisms of flow regime transitions. This research is part of a joint program between the University of Wisconsin-Milwaukee (Kojasoy) and Purdue. The research program is expected to lead to a) local instrumentation methods for detailed interfacial characteristics, b) data for local interfacial area, void fraction, relative velocity and wave propagation velocity, and c) phenomenological models for interfacial area and interfacial structural changes.

\section{Rensselaer Polytechnic Institute Dept of Mechanical Eng, Aeronautical Eng Mechanics Troy, NY 12180-3590 \\ $\$ 122,000$ \\ 01-B \\ 89-4}

\section{Uitimate Limits of Boiling Heat Fluxes}

\section{A. Bergles, M. Jensen}

This study is directed toward the thermal-hydraulic behavior of water and aqueous mixtures, flowing in plain tubes and in tubes with enhancement devices, at very high heat fluxes. The mode of heat transfer is subcooled nucleate boiling, and the limiting phenomenon is the critical heat flux (CHF). Very large heat fluxes can be accommodated on a steady basis with pure water by use of large subcoolings, high velocities, small tube diameters, and short tubes. It is expected that simultaneous use of several enhancement techniques will extend the maximum heat flux to at least $5 \times 10^{8} \mathrm{~W} / \mathrm{m}^{2}$, which would be higher than reported in any study to date. The wall temperature characteristics, usually presented as a boiling curve, are unknown at such high heat fluxes.

An experimental program has been designed to systematically investigate the effects of subcooling velocity, tube geometry, and enhancement techniques on CHF, the boiling curve, and the pressure drop. The CHF's will be correlated as a function of flow and geometrical variables. The experiments will be complemented by mechanistically based models for CHF under normal and enhanced conditions.

The experimental facility has been modified to permit data acquisition under extreme conditions of flow and heat flux. CHF data have been obtained with pure water flowing in plain tubes to describe the main parametric trends. A complementary data base of results from the literature is being assembled for the correlation development.

\section{Rensselaer Polytechnic Institute Dept of Mechanical Engineering, $\$ \$ 128,000$ Aeronautical Eng \& Mechanics 01-A Troy, NY 12180-3590 91-3}

\section{Inelastlc Deformation and Damage at High Temperature \\ E. Krempl}

A combined theoretical and experimental investigation is performed to study the biaxial deformation and failure behavior of AISI Type 304 Stainless Steel under low-cycle fatigue conditions at elevated temperature. The purpose is to characterize the material behavior in terms of mathematical equations which are ultimately intended for use in inelastic stress analysis and life prediction. Creep-fatigue interaction and ratchetting are of special concern. The long-term 
goal is the development of a finite element program that can directly calculate the life-to-crack initiation of a component under a given load history.

For the experiments, an MTS servohydraulic axial/torsion test system is available together with an MTS Data/Control Processor. Induction heating ( $10 \mathrm{kHz}$ frequency), MTS biaxial grips and an MTS biaxial extensometer are available :ogether with a reversing direct current potential drop facility. It is intended for early monitoring of damage during cyclic loading. It has been tested out at room temperature.

Uniaxial and torsional ratchetting experiments showed considerable strain accumulation at room temperature and they demonstrate that ratchetting is due to viscous effects. Surprisingly, insignificant ratchetting and rate sensitivity were observed at 550,600 and $650^{\circ} \mathrm{C}$ for uniaxial tests. This unexpected finding was attributed to strain aging in the stainless steel. A finite deformation theory of viscoplasticity based on overstress (VBO) is being developed and is being implemented into a finite element computer program. To simulate the complex hardening behavior of AISI Type 304 Stainless Steel during non-proportional loading. new hardening rules are under investigation and are being implemented into the small deformation VBO theory developed previously.

\section{Rensselaer Polytechnic Institute Dept of Nuc Eng \& Eng Physics $\$ \$ 122,000$ Troy, NY 12180-3590 06-C $89-4$}

The Continuum Modeling of Two-Phase Systems

R. Lahey, Jr., D. Drew

The primary objective of the research being conducted is to develop a mathematically consistent multidimensional two-fluid model which agrees with data and satisfies all the constraints implied by physical laws and the postulates of continuum mechanics.

To this end, interfacial closure laws are being developed such that two-fluid models can be used to predict many important phenomena, such as the evolution of interfacial area density and flow regime transition. Void wave phenomena are also being investigate $J$, both analytically and experimentally, in order to assess and thoroughly understand the effect of the proposed closure laws on the void wave eigenvalues of the two-fluid model (i.e., to study well-posedness).

It is intended that the results of this research will significantly advance the state-of-the-art in the two-fluid modeling of two-phase flows such that, in the future, multidimensional two-fluid models can be used with confidence for many problems of practical interest.

\section{Rensselaer Polytechnic Institute Dept of Chemical Engineering $\$ \mathbf{\$ 8 3 , 7 1 5}$ Troy, AIY 12180-3590 \\ $01-\mathrm{C}$ 89-3 \\ Microcomputer Enhanced Optical Investigation of Spreading and Evaporation Processes in Uitra-Thin Films P. Wayner, Jr.}

The physicochemical phenomena associated with fluid flow and change-of-phase heat transfer in ultra-thin (thickness less than $10^{-5} \mathrm{~m}$ ) liquid films are being studied. Microscopic image-processing equipment, procedures, and related computer programs are being developed to improve data resolution and automate data acquisition. First, the image processing equipment are being developed and used in conjunction with an interferometer designed to study the transient film thickness in draining and evaporating films. The glass cell is designed to optimize temperature control, cleanliness and simplicity. The optical data is obtained using a video camera attached to a microscope through which the interference fringes are recorded. Transport processes in polar and non-polar fluids, with and without heat transfer. are being experimentally studied and analyzed. Using the results of these studies, an ellipsometer with a complementary interferometer has been designed and is being constructed. The long term objective is to determine the heat transfer characteristics of evaporating ultra-thin films. The near term obisctive is to develop microscopic image-processing equipment and a complementary heat transfer cell. 


\section{Rice University \\ Dept of Clvil Englneering \\ Houston, TX 77251}

$\$ 81,034$
$01-A$
$91-3$

Stochastic Quadrization for Probabilistic Analysis of Large Compllant Off-Shore Structures

\section{P. Spanos}

The objective of this research is to study the nonlinear probabilistic dynamics of large structures subjected to nonlinear forces in general, and the probabilistic analysis of offshore marine structures subjected to nonlinear drag forces in particular.

Almost all structures used in engineering practice are subject to random physical stimuli and respond with noninear behavior. This is true with energy production systems such as offshore structures subjected to ocean waves. In designing such structures, the engineer faces problems of almost unsurmountable complexity. Thus, it is very important to develop methods of analysis to treat problems involving randomness and nonlinearity. The methods in existence today are highly approximate and over-simplified. With the level of complexity and generality which exist in current energy structures, such as the tension leg platform for offshore oil drilling, current methods of analysis and design are unwieldy and inadequate, if at all valid. A method of analysis is needed which features simplicity and the accuracy of nonlinear response.

In the proposed research, a method of analysis is to be developed that efficiently addresses problems in nonlinear probabilistic dynamics in general, and the analysis of offshore structures in particular.

\section{University Of Rochester Dept of Physics and Astronomy Rochester, NY 14627}

Flux Flow, Pinning, and Resistive Behavior in Superconducting Networks

\section{S. Teitel}

The motion of vortex structures, in response to applied currents, is a major source of resistance in superconducting networks in magnetic fields. Systems of interest include regular Josephson junction arrays and type II superconductors, such as the new granular high Tc ceramics. Numerical simulations of finite temperature, current carrying, networks will be carried out to provide a characterization of vortex response in nonequilibrium situations. For periodic networks, current-voltage (I-V) characteristics will be computed and compared with experimental results. The effects on resistivity of transitions from pinned to unpinned or to melted vortex structures, will be investigated. For disordered networks, the effects of pinning in producing metastable vortex structures leading to glassy behavior will be explored.

To date, simulations have been carried out for two dimensional regular Josephson junction arrays, as well as arrays with random vortex pinning sites. Various values of applied magnetic field have been studied and $\mathrm{I}-\mathrm{V}$ characteristics and resistivity were computed and related to vortex structure. We have also simulated the equilibrium behavior of fluctuating vortex lines in a three dimensional network, as a model for high Tc superconductors. Melting of the vortex line lattice into a line liquid, and properties of the line liquid, have been investigated and compared with recent theoretical results.

This research will greatly enhance our fundamental understanding of behavior in strongly fluctuating superconducting materials. The results will have impact in understanding the magnetic properties of the new high Tc superconductors, and in the design of Josephson junction arrays for use as microwave detectors and generators.

\section{University Of Rochester}

Dept of Physlcs and Astronomy

Rochester, NY 14627

$\$ 89,667$

$06-\mathrm{C}$

Coherence Effects in Radiative Energy

90-3

\section{Transfer}

E. Wolf

The main objective of this research is the elucidation of how coherence properties of sources affect the spatial and the spectral distribution of energy in optical fields. The clarification of such questions is of importance for the theory of radiative energy transfer and for radiometry with sources of different statistical properties. The influence of sourcecorrelations in the physical parameters of random media with which a field 
may interact on the spectral properties of the scattered radiation is also being exammed.

The progress made so far includes the clarification of the relationship between the radiance of a field produced by a class of partially coherent optical sources and the radiance (specific intensity) of the phemomenological theory; elucidation of effects of diffracting apertures on spectra of partially coherent fields; and formulation of laws for propagation of the radiance through paraxial optical systems.

Current research includes the formulation of new conservation laws in which spectral changes take place and investigations of spectral modifications produced in multiple scattering. The possibility of determining correlation functions of media from observed spectral changes of scattered radiation is also being examined.

\section{The Rockefeller University}

\section{Department of Physics}

1230 York Avenue

New York, NY 10021

$06-\mathrm{C}$

88-3

\section{Some Basic Research Problems Related to Energy \\ E. Cohen}

The present project is concerned with the following problems. 1) The approach to thermal equilibrium of dynamical systems with relatively few degreesof-freedom is studied. This is of interest not only for the foundations of statistical mechanics but also for chemical and other problems. The question is: when does equipartition of energy and other thermal equilibrium properties hold? Computer as well as theoretical calculations will be used. 2) An investigation of Lorentz lattice gas cellular automata with independent particles moving on a lattice occupied by randomly placed scatterers is carried out. Two goals are a closer study of (a) the abnormal diffusion found in strictly deterministic models and, (b) the connection with polymer statistics. Computer and analytic approaches will be employed. This investigation is also expected to elucidate the connection between particle motion in lattice gas cellular automata and the hydrodynamic behavior of fluids in general. 3) A possible relation between the transport coefficients of a fluid in ordinary space and the Lyapunov exponents of the same system in phase space will be investigated using computer simulations as well as theory. 4) A newly discovered analogy between :.ie structural relaxation found in dense atomic fluids and that in concentrated solloidal suspensions will be further explored. Laboratory experiments, computer simulations as well as analytical methods will be used.

\section{Sandia National Laboratories Fluid, Thermal, and Structural Sciences Division Albuquerque, NM 87185

\section{Two-Phase Flow Measurements by NMR R. Givler}

This project will develop nuclear magnetic resonance (NMR) methods to measure velocity and concentration distributions of both phases in two-phase flows. Systems to be studied include concentrated suspension flows in circular tubes, solid-liquid flows in Couette geometries as well as to gas-liquid and liquid-liquid flows.

One approach to velocity measurements is to measure an incremental spatial displacement of nuclear spins that are noninvasively tagged whereas another is to measure the phase evolution of the nuclear spin precession which depends on the translational velocity. The first method is best suited for flows in uniform geometries such as pipe flows whereas the second, more ccimplex method, is better suited for non-uniform cases such as for nonneutrally buoyant flows in a Couette geometry.

In order to improve the capabilities of the apparatus to make rapid measurements, a major instrumentation upgrade is being performed during summer and fall of 1991 . This consists in a new operating system and computer, hardware improvements to the if electronics, and a shielded magnetic fierd gradient coil system. The studies of concentrated suspensions in pipe flow for velocity and concentration distributions is complete except for measurements of particle velocities. The next major experimental emphasis will be on liquidsolid flows in an asymmetric Couette device.

This work is carried out in collaboration with the Lovelace Medical Foundation. 


\section{Sandia National Labaratories Combustion Research Facility Thermofluids Division \\ Livermore, CA 94550 \\ $\$ 143,000$ \\ 06-B \\ 90-3}

Mixing and Phase Change During Combustion

\section{A. Kerstein}

The principal focus of this research program is the development of a turbulent mixing model applicable to combusti $n$ flows. In work to date, a novel computational approach has been formulated in which turbulent stirring is represented by non-linear mappings on a line, iterated according to a stochastic rule that incorporates key aspects of the Kollisgorov cascade picture of turbulence. Computations and analysis demonstrate substantially better predictive capability than achieved by previous models of molecular mixing in turbulent flow. Future work will involve further analysis of the mathematical origins of the computed results, refinement of the model to capture subtle effects such as the influence of molecular diffusion on turbulent transport, and application to unsolved problems of turbulent mixing in non-reacting turbulent shear flows and turbulent combusting flows.

Aspects of heterogeneous combustion are also addressed in this program. Stochastic networkbreakup models are applied, on a macroscopic scale, to the morphological evolution of a reacting porous solid, and on a microscopic scale, to the thermochemical dissociation of a macromiolecular fuel such as coal.

\section{Sandia Naitional Laboratories Combustion Research Facility Livermore, CA 94551-0969 \\ $\$ 123,500$ 06-B 90-3}

Structure of Inverse Diffusion Flames in Supercritical Fuel/Water Mixtures

\section{R. Steeper}

This research project investigates the stru ture of inverse diffusion flames in supercritical fuel/water mixtures. Inverse diffusion flames have received very little attention in the literature althouyh they possess unique characteristics that are applicable to chemical processing and materials synthesis. The focus of this project is the specific class of iniverse diffusion fiames in which a laminar jet of oxidizer is introduced into a background of hydrocarbon fuel and water at a temperature and pressure above the thermodynamic critical point of the fuel/water mixture. These flames have direct application to the destruction of aqueous and mixed hazarcious wastes, but little is currently known about the ignition, extinction, or stability of flames at high pressures, particularly flames at supercritical water conditions. Sandia has built a laboratory-scale supercritical reactor and demonstrated the capability to obtain quasi-steady inverse diffusion flames in supercritical mixtures of water and methane. The reactor provides optical access to the reaction zone, and a mapping of temperature, species, and velocity fields is planned. Both analytical and numerical models of laminar diffusion flames are available to support the experimental efforts, however these models must be modified to include a supercritical fluid eqiation of state and supercritical transport properlies.

\section{University Of Southern California}

$\begin{array}{lr}\text { Department of Mechanical Engineering124,514 } \\ \text { Los Angeles, CA 90089-1453 r } & 01-C \\ & 91-3\end{array}$

Particle Pressures in Fluidized Beds

\section{Campbell}

The particle pressure represents the portion of the momentum transport in a fluidized bed that can be attributed to the motion of particles and their interactions. Such forces are responsible for the attrition of fluidized particles and postulated behaviors of the particle pressure have been used as a mechanism to explain the stability of homogeneously fluidized beds. But, until recently, there have been no measurements of the particle pressure. Preliminary measurements, in gasfluidized beds, have shown that the particle pressures exerted on the side walls are primarily due to the presence of bubbles and that the magnitude of the average particle pressure scale with the bubble size. The purpose of the present investigation is to continue this work to better understand the mechanisms that lead to particle pressure generation in both gas and liquid fluidized beds. Both investigations will be performed in two-dimensional beds. In the gas bed, the goal is to determine the distribution of particle pressure around an injected twodimensional bubble as a way of understanding the 
distribution of particle pressure across the bed. The studies in the liquid bed will be used to try and understand the behavior of the particle pressure near the point of initial instability of the bed and the pressure that are generated in the early stages of unstable behavior. Funding is scheduled to begin $9 / 1 / 91$.

\section{Southwest Research Institute} 6220 Culebra Road

San Antonio, TX 78228-0510

$\$ 99,912$

03-B

91-3

Application of Magnetomechanical Hysteresis Modeling to Magnetic Techniques for Monitoring Neutron Embrittlement and Blaxial Stress

M. Sablik

The objective of this project is to study experimentally and theoretically the effects of neutron embrittlement and biaxial siress in steels on signals from various magnetic measurement techniques. It is expected that interaction between experiment and modeling will result in the design of efficient magnetic measurement procedures for monitoring neutron embrittlement and biaxial stress.

Magnetic measurement techniques to be assessed are: (1) magnetic hysteresis loop measurement of properties like coercivity and permeability; (2) magabsorption, which involves measurement of the impedance of an if coil brought close to a magnetic sample; (3) Barkhausen noise analysis; (4) magnetically induced velocity change (MIVC) of an ultrasonic wave; and (5) higher order harmonic analysis of an ac magnetic hysteresis loop. The model of Sablik et al for magnetic hysteresis and uniaxial stress effects on magnetic properties will be extended to conditions of biaxial stress and neutron embrittlement. The effects of these consitions on magnetic probe signals (I) - (5) will be modeled and compared to experiment. In the case of neutron embrittlement, measurements will be made on steel samples previously characterized by Charpy tests after being exposed to various neutron fluences.

Results from this project should ultimately assist in safety monitoring in the nuclear power and gas industries.

\section{Stanford University}

Dept of Mechanicai Englneering \$0

Stanford, CA $94305-3030 \quad 01-B$

89-3

Heat Transfer in Three-Dimensional

Turbulent Boundary Layers

J. Eaton

The objectives of this research are to identify, understand, and model the effects of mean flow three dimensionality on turbulent boundary layer heat transfer. Our previous work has shown tha! vertical mixing through the boundary layer is suppressed by three dimensionality and that the wall heat transfer coefficient is reduced. Experiments on embedded longitudinal vortices have led to the hypothesis that turbulent mixing is reduced in the boundary layer because naturally occurring longitudinal vortices deep in the boundary layer are attenuated by the crossflow. The effects are expected to be asymmetric in that vortices of one sign are attenuated very rapidly while the effects of opposite sign vortices are more persistent.

The present experiments are examining both the heat transfer and turbulence structure on a large disk rotating in a quiescent environment. Single point turbulence measurements have shown that the turbulence mixing is suppressed as in other 3D boundary layers. Multipoint correlations with radial separations show a substantial asymmetry supporting our hypothesis. A constant heat flux disk for accurate measurement of the heat transfer coefficient and the turbulent heat flux is now being fabricated.

\section{Stanford University}

Dept of Mechanical Engineering \$97,035

Stanford, CA 94305-3030

$01-A$

91-3

Stabllity and Stress Analyses of Surface

Morphology of Elastic and Piezoelectric Materials

\section{H. Gao}

The goal of this research is to investigate the mechanical effects of surface morphology of elastic dielectric and piezoelectric materials. In particular, the project will study the stability of a flat surface against diffusional perturbations and the stress concentration caused by slightly undulating surfaces. 
The surface morphology of materials will be studied by using a unified perturbation procedure based on the notion of thermodynamic forces and the energy momentum tensor. The thermodynamic forces on material inhomogeneities such as interfaces and inclusions are a measure of the rate at which the total energy of a physical system varies with the configurational change of these inhomogeneities. Within the general methodology, any type of material and loading condition can be studied as long as the proper forces can be identified. By using corresponding material conservation laws discovered previously, a systematic analysis of surfaces of piezoelectric solids will be made. Preliminary studies have shown that under sufficiently large stresses, surfaces of materials become unstable against a range of diffusional perturbations bounded by two critical wave lengths. Even a slight undulation caused by these unstable diffusional perturbations, such as micro-level bumps and troughs, can result in a significant stress concentration along a material surface. These concentrations may lead to mechanical failures along the surface and may have more consequences for piezoelectric materials where the deformation is coupled to an applied electric field. There are also suggestions that the stress distributions in a body may be sensitive to the surface morphology.

\section{Stanford University \\ Dept of hechanical Engineering $\$ \mathbf{\$ 1 7 7 , 4 4 0}$ Stanford, CA 94305-3030 \\ Energy Changes in Transforming Solids G. Herrmann, D. Barnett}

In mechanics of solids, defects may be modeled as inclusions or holes and energy changes play a preponderant role in characterizing a variety of processes. The heterogenization technique recently developed with DOE support is most useful in this context. It has been further extended, providing several universal formulas, e.g. for the average stress between two holes or inclusions, for inclusions with imperfect interfaces and for stresses in cylindrical and plane layered media. Based on newly found conservation laws, interaction forces between two inclusions (defects) have been calculated. A new methodology to establish conservation laws for dissipative systems has been advanced, and a theory of stressed solids prone to damage is being reformulated on the basis of the more appropriate Gibbs free energy.

A portion of our research has as its objective the development of further understanding of subsonic and supersonic surface waves and interfacial waves as well as of bulk waves in anisotropic linear elastic solids. In the past year we have shown that it is indeed possible for onecomponent surface waves (surface waves consisting of a single partial wave or inhomogeneous plane wave) to exist in stable solids and have constructed examples of such waves. These waves are necessarily supersonic and are polarized parallel to the boundary of the half-space in which they exist. In addition, we have shown that subsonic two-component surface waves (formed from two partial waves), which are known to exist in real crystals, can never be polarized parallel to the half-space boundary. We are currently developing a numerical scheme for detecting supersonic surface waves and spaces of "simple reflection" in real crystals.

\section{Stanford University}

$\begin{array}{lr}\text { W.W. Hansen Lab of Physics } & \$ 0 \\ \text { Stanford, CA 94305-3030 } & 03-B \\ & 90-3\end{array}$

\section{Nondestructive Testing}

\section{G. Kino}

The aim of this project is to develop a photothermal microscope for non contact testing of materials. Techniques of this kind are particularly well suited to the determination of thermal parameters, and anisotropy of small samples.

One example of the work is the measurement of high temperature superconductors over a range of temperatures from room temperature through the critical temperature $T_{C}$ down to $20^{\circ} \mathrm{K}$. A modulated laser beam, focused to less than $1 \mathrm{~m}$ diameter, impinges through a sapphire window onto a sample of $\mathrm{Bi}-\mathrm{Ca}-\mathrm{Sr}-\mathrm{Cu}-\mathrm{O}$ in a helium cryostat and periodically modulates its temperature. This process excites a thermal wave, which can be detected by the variation in reflected signal amplitude of a second focused laser beam, due to the change of refractivity with temperature. The sample can be rotated under the beams and the thermal diffusion coefficient, its anisotropy and its magnitude can be measured from the phase delay of the thermal wave. By measuring the amplitude 
of the thermal wave, material phase changes associated with superconductivity can be measured. A pronounced peak in amplitude is seen at the critical temperature $T_{C}$. Even stronger effects of this type are observed with charge density waves in a variety of materials.

\section{Stanford University}

Dept of Clvil Engineering

Environmental Fluid Mechanics Lab Stanford, CA 94305-4020
$\$ 192,040$

$01-B$

90-3

\section{Fluid Dynamics of Double Diffusive Systems}

\section{J. Koseff, R. Street, S. Schladow}

This project is focused on processes that occur in a double-diffusive system subject to external forcing, using a combined experimental and numerical approach. In particular it addresses a water body that is continuously stratified in both temperature and salinity. The forcing is provided by a combination of a lateral heat flux and a surface shear. Double-diffusive instabilities and mixed layer deepening occurs in response to this forcing, resulting in changes to the vertical and horizontal fluxes of heat and salt. The goals are to (1) understand the initiation and evolution of the double diffusive instabilities, (2) quantify the effects on the vertical fluxes, and (3) compare observations of the mixed layer deepening to the existing data for singly stratified systems. The experimental component has to date concentrated on the effects of lateral heating. A new $4.0 \mathrm{~m}$ long and $0.8 \mathrm{~m}$ wide experimental tank, together with appurtenant facilities for controlling the initial stratification, has been commissioned. The end wall of this tank is uniformly heated using a radiant source. Fast response thermistor and conductivity probes are used to provide vertical profiles, and flow visualization is provided by video imaging of dye in illuminated planes. Initial findings suggest that the nature of resulting convective intrusions is a function of two dimensionless stability parameters. The numerical component involves using a finite volume formulation of the non-linear Navier-Stokes, conservation of mass, and conservation of species equations in three space dimensions and in time. The experimental results suggest that the high resolution required to satisfactorily represent the intrusion interfaces will make a conventional modeling approach very difficult, with the number of grid points required to resolve the interfaces excessive, even in the context of modern supercomputers. For this reason work on implementing an adaptive gridding procedure has been commenced.

Adaptive gridding allows the grid to be selectively refined only in those areas where high resolution is required, thus facilitating large savings in both computational time and memory requirements.

\section{Stanford University \\ Mechanical Engineering Dept $\$ \mathbf{\$ 2 5 3 , 1 6 0}$ Stanford, CA 94305 \\ 03-B \\ 88-4 \\ Diagnostics for Plasma Chemistry \\ C. Kruger, M. CappellI}

This research is concerned with optical diagnostics for plasma chemistry and plasma processing, with an emphasis on methods which allow for departures from local thermodynamic equilibrium -- such as finite chemical reaction rates, nonequilibrium electron densities and temperatures, and radiation loss effects. Laser and other optically based methods are being developed for measurements of plasma parameters including electron density and temperature. The methods under study are designed to be applicable whether or not the plasma is in local thermodynamic equilibrium, and indeed to assess the importance of nonequilibrium effects under conditions of interest in plasma chemistry.

Results with an induction plasma facility show significant nonequilibrium at the exit of a downstream quartz test section and suggest possible errors in conventional diagnostics assuming local thermodynamic equilibrium. Measurements of the radiation source strength in argon indicate an order-of-magnitude difference from values reported earlier at temperatures of interest in plasma processing.

To apply this approach to diagnostics to a realistic and promising form of plasma processing, we have undertaken experiments on diamond synthesis on substrates in the test section of the induction torch. Diamond crystals and films have been grown at rates that are at least one order of magnitude greater than those characteristic of low-pressure diamond synthesis. 
Stanford University

Dept of Chemlstry

Stanford, CA 94305

$\$ 135,000$

06-C

89-3

Thermodynamics in Combustion and Engines J. Ross

Research is concerned with the issue of enhancement of power output in thermal and chemical engines by means of external perturbations of constraints coupled to nonlinearities of the mechanism of the engine. The theoretical possibility of an increase in power output of a thermal engine driven by a chemical reaction by means of external periodic variations of reactant influx has been confirmed in a series of experiments. The power output of an engine is necessarily accompanied by dissipation due to irreversible processes essential for power production. Hence an increase in power output by means of external perturbations usually implies a decrease in dissipation but may also come about due to a change in the final state of the system. Increases in efficiency can be achieved for a particular range of frequencies and amplitudes of external perturbations which yield resonance effects and appropriate phase shifts of fluxes and forces. Theoretical and experimental studies are in progress on optimization of efficiency by choices of functional forms of external perturbations. A variational theory has been constructed which gives limits of improvements in efficiency by external perturbations.

Research is in progress on the construction of a thermodynamic and stochastic theory of irreversible processes far from equilibrium. The theory has been completed for single and multivar able systems with single and multi-variable stationary states.

\section{Stanford University \\ Dept of Mechanical Engineering \\ Stanford, CA 94305}

$\$ 80,000$ 03-A 88-3
Programming to bear on finite element optimization problems related to mechanical component shaping. Better analysis, approximation and computation methods would contribute to the design of lower weight, more energy efficient vehicles and machines. Since for existing methods each iteration requires a computationally intensive finite element analysis (FEA), these innovations are intended to reduce the number of FEA iterations.

This approach has been applied successfully to the shaping of a planar cantilever support for minimum weight. Three zones: (1) the fillet near the support, (2) an interior zone free of stress concentration, and (3) one near the load -- were modeled approximately by classical solid mechanics and more precisely by FEA. Monotonicity analysis of the classical approximation identified as critical the shear stress near the load and the bending stress in the fillet and interior zones. This immediately determined the optimal shape of the interior and load zones. It also gave the differential equation of the fillet shape as a function of its variable curvature, initially unknown. FEA-predicted deviations of fillet stress from the desired value generated a new curvature function whose integration produced a much better shape. Only three iterations were needed for convergence. The idea is also proving practical for deflection- and vibration-limited cantilever situations. A connecting rod vibration study has shown remarkably large shape improvement in a single iteration.

Nearing completion is another study of computational stability in the geometric modeling of curved surfaces. It has been established that reproducibility after rotation and other motions is greatly improved by slight relaxation of interpolation restrictions. This allows compromise between the two often conflicting parametric placements of the control points. A new universal quadrilateral patch has been developed which will permit important simplification of scultured surface codes.

Monotonicity Analysis and Geometric

Programming in Mechanical Shape

Optimization

D. Wilde, S. Sheppard

This theoretical and computational research intends to bring the theory and practice of Monotonicity Analysis and Geometric 


\section{Stevens Institute Of Technology Dept of Physics and Engineering \$0 Hoboken, NJ 07030 06-C \\ 87-4}

Investigation of Transitions From Order to Chaos in Dynamical Systems

G. Schmidt

Coupled nonlinear oscillator systems are of great interest. They describe diverse phenomena such as coupled $p-n$ junctions, Josephson junction arrays, and serve as approximations of nonlinear partial differential equations, describing the onset of fluid turbulence, reaction diffusion systems, etc.

We have found recently, using renormalizationgroup treatment, that large classes of such systems have universal properties, i.e. as the parameters of these systems are varied, qualitative as well as quantitative predictions can be made as to the change of behavior, bifurcations, onset of chaos etc.

Having performed the abstract analysis, we are now in the process of applying it to study the behavior of concrete coupled systems, utilizing and checking the general results we obtained.

\section{Syracuse University Dept of Chemical Engineering and Materials Sclences Syracuse, NY 13244-1190}

\section{Numerical and Physical Modelling of Bubbly Flow Phenomena \\ A. Sangani}

The objective of the project is to understand how the microstructure of bubbly liquids, i.e., the details of the size, shape and spatial distributions of bubbles, depend on the nature of flow and how the overall properties of the flow in turn depend on the nature of microstructure. $A$ better understanding of this interdependence is crucial in formulating reliable models for the flow of bubbly liquids. The work during the past year has focused on flows that are either oscillatory in nature as in the propagation of pressure or acoustic waves in bubbly liquids or that are nearly homogeneous as in kinematic waves in nearly uniform flow of bubbly liquids. It was found that the properties of the bubbly liquids and the resulting microstructure in the two flows are very different. The present efforts are directed towards dieveloping fast algorithms for numerically solving the multi-bubble interactions.

\section{University Of Texas At Austin Ctr for Studies In Statistical Mech $\$ \mathbf{\$ 1 0 0 , 0 0 3}$ and Complex Systems Austin, TX 78712 $06-\mathrm{C}$ 91-3}

The Behavior of Matter Under Nonequilibrium Conditions: Fundamental Aspects and Applications

I. Prlgogine, T. Petrosky

This proposal, which is a continuation of previous work deals with the behavior of matter under nonequilibrium conditions in terms of microscopic description. A theoretical framework for integrating "non-integrable" large Poincare systems will be applied to the study of a number of interesting situations. (1) Application to interacting fields, including classical and quantum electrodynamics: The method permits the representation of radiation damping as part of the complex spectrum of the Louville operator, and avoids the well-known difficulties of the classical and quantum radiation damping theory. (2) Numerical validation of the approach: Numerical experiments will be pursued in order to verify the quantum formulation. Especially, the collapse of the wave function and the appearance of an equilibrium statistical distribution, as predicted by the theory, will be verified. (3) The present work, already well advanced for the definition of unstable particles in the frame of a complex spectral representation, will be continued. Unstable particles with decayin y modes cannot simply be identified, since decaying modes, taken separately, do not carry energy. (4) The theory gives the possibility to derive new criteria for the transition to classical chaos by studying the complex spectrum of the Liouville operator in a form that is appropriate to mapping probiems. (5) The theory goes beyond the nonMarkovian master equation and permits a new approach for the study or transport processes (diffusion, viscosity, etc.). (6) Irreversibility appears to be of special interest in high-energy problems, and especially to transitions from virtual to real particles. 


\section{University Of Texas At Austin}

Dept of Physics

Austin, TX 78712

$\$ 144,777$

06-C

87-5

Complex Temporal and Spatial Patterns in Nonequilibrium Systems

H. Swinney

Dynamical systems methods are being developed and used to characterize the formation and evolution of temporal and spatial patterns in systems maintained far from equilibrium. In particular, experiments and analyses are considering electrodeposition of fractal metallic clusters, pattern formation in reaction-diffusion systems, and instabilities in convecting fluids. Novel reactors have been developed to search for chemical patterns (i.e., spatial variations in the chemical composition), and sustained patterns have been found in several different one- and two-dimensional geometries. Bifurcations in these patterns are studied by varying control parameters, e.g., the concentrations of the feed chemicals or the temperature. The observed two-dimensional chemical patterns range from the stationary patterns, similar to those predicted by Turing in 1952 but not observed until 1990, to chemical turbulence, which is characterized by large numbers of defects and a rapid decay of spatial correlations. Much needs to be done, both experimentally and theoretically, to characterize and understand these spatiotemporal patterns. These laboratory experiments together with numerical and analytic studies of models should provide general insights into the formation and evolution of patterns in nonequilibrium systems.

\section{Tufts University}

Dept of Mechanical Engineering $\$ 0$ Medford, MA 02155 01-A

Effective Elastic Properties of Cracked Solids M. Kachanov

The knowledge of effective elastic properties of solids with cracks appears to be of increasing engineering importance. Extensive microcracking in structural elements working under conditions of high temperatures or irradiation, and microcracking in composite materials under fatigue conditions may noticeably reduce the stiffness of the material and make it anisotropic. Understanding and prediction of these changes are essential for proper design and strength and lifetime assessments. A new approach to many cracks problems based on interrelating the average tractions on individual cracks is introduced. Its advantages are that it yields simple analytical results which are quite accurate up to very high crack densities and that it can be applied to crack arrays or arbitrary geometry. Relation between deterioration of elastic properties and "damage" is discussed.

\section{United Technologies Res Center Propulsion Sclence Lab East Hartford, CT 06108 $\$ 175,278$ 03-A 89-3}

\section{Laser Dlagnostics Of PACVD Processes} W. Roman, S. Hay, F. Otter, A. Eckbreth

The research objective is the comprehensive experimental investigation of the fundamental nonequilibrium reactive plasma assisted chemical vapor deposition (PACVD) process as applied to hard face coatings (e.g. $\mathrm{TiB}_{2}$ or diamond). Nonintrusive laser diagnostics (e.g. laser induced fluorescence (LIF) and coherent anti-Stokes Raman spectroscopy (CARS)) are being used to probe gas phase species, concentrations and rotational temperatures in situ. Detailed coating characterization is accomplished using Auger, Ion Scattering and secondary ion mass spectroscopies (AES, ISS and SIMS) and complementary techniques. In addition, coating characteristics such as smoothness, adhesion (UTRC custom built pin-on-disc apparatus) and hardness (stateof--the-art nanoindenter apparatus) are measured. Gas phase spectroscopy is interpreted through chemical kinetic modelling and will be correlated to coating characteristics thus providing a predictive capability that is severely lacking in the present science base of advanced protective coatings. These techniques are also applicable to other processes such as PVD, CVD, combustion and thermal plasma deposition. Results to date include:

1) fabrication of a $5 \mathrm{~kW}$ if PACVD reactor system

2) exploratory spectral emission studies

3) development of substrate preparation technology and characterization

4) implementation of a ultramicrohardness tester and adhesion test apparatus and analysis of coating hardness and elastic modulous 
5) coating durability testing using UTRC erosion test facility

6) initial characterization of $\mathrm{TiB}_{2}$ and diamond coatings

7) first tire CARS observation of diborane $\left(B_{2} \mathrm{H}_{6}\right)$ in a PACVD process

8) in situ axial concentration and temperature profiles of key species (diborane and $\mathrm{H}_{2}$ )

9) formulation of chemical kinetic models to account for spectral observations

\section{Washington University}

Department of Systems Science

and Mathematics

Saint Louis, MO 63130

$\$ 72,755$

03-A

90-3

Dynamical Systems with Internal Structure: A New Approach to the Problem of Analysis and Robust Design of a Time Varying System B. Ghosh

The main objective of the proposed project is to study design problems for linear time-varying control systems leading to a robust design via an adaptive controller. Specifically, for autoregressive moving average systems with time-varying coefficients, it turns out that a good design strategy is to adaptively assign the parameters of the closed loop system by a judicious choice of a time-varying compensator. The parameters of the compensator which assign a specified set of coefficients in the closed loop satisfy a nonlinear recursion. We propose to study these recursions in details. For specific cases, we show that the proposed recursion is an Anosov diffeomorphism on a three-dimensional torus. In particular, such a dynamic is chaotic. We feel that our design should try to avoid chaotic dynamics.

In order to analyze and design a compensator for a time-varying system it is important to be able to estimate the trend, i.e., predict future values of the parameters of the system. An important part of our project is to assume that the parameters change in accordance with an internal structure viz. a time series. Our goal is to estimate the parameters of the internal structure.

Thus we propose to investigate in this project an identification scheme with internal structure together with recursive coefficient assignment in real time in order to generate a desirable performance in the closed loop.
Washington State University Dept of Mechanical Engineering $\$ 69,000$ Pullman, WA 99164-2920

91-2

\section{Mass and Energy Coupling Effects in Multiphase Free Shear Flows T. Troutt}

The primary goal of this research is to determine the role organized vortex motions play in the dispersion of particles by free shear flows. This research goal has been pursued using analytical, numerical and experimental techniques to examine the particle dispersion process in plane mixing layers and wakes. The results of these studies have shown that the character of the particle dispersion process in free shear flows is a function of a time scale parameter formed between the ratio of the particle aerodynamic response time and the time scale of the organized vortex motion. For intermediate time scale ratio particles two types of dispersion mechanisms have been recently identified. In mixing layers, where large scale vortex mergers occur regularly, intermediate scale particles are concentrated into sheet like patterns which undergo stretching and folding operations. For plane wakes, where large scale vortex mergers are not a dominant feature, intermediate scale particles are "focussed" into highly concentrated regions near the vortex boundaries. The resulting order of the self-organized particle dispersion patterns for both mixing layers and wakes can be quantified using the fractal correlation dimension. This quantity is found to be a strong function of particle time scale ratio. Current research efforts are focussed on exploring the effects of two-way mass, momentum and energy coupling between the particulate phase and the carrier fluid. These multiphase coupling effects may have significant importance with regard to predicting the performance of many energy conversion systems. New design concepts for multiphase mixing systems are a highly plausible application of these results. 
University Of Wisconsin Mechanical Engineering Dept Mllwaukee, WI 53201

Interfaclal Area and Interfaclal Transfer In

Two-Phase Flow Systems

\section{G. Kojasoy}

The objective of the research program is to develop instrumentation methods, experimental data base and analysis leading to predictive models for describing the interfacial structure and behaviors of two-phase flows. In terms of the flow structure, the local void fraction, interfacial area concentration, fluid particle size distribution and flow patterns in vertical two-phase flow systems are studied in detail. For the purpose of understanding the dynamic behaviors, the interfacial velocity, wave characteristics and coalescence and disintegration of fluid particles are investigated. Special emphasis is placed on developing the four-sensor and five-sensor resistivity probe method and improving the double-sensor resistivity probe method. A second emphasis is on the system size and entrance effects on the interfacial characteristics described above. The changes in the interfacial structures and flow regimes as a function of an axial location are measured experimentally. This information together with the local measurements from the probe method are used to study the mechanisms of flow regime transitions. This research is part of a joint program between Purdue University (Ishii) and the University of Wisconsin-Milwaukee. The research program is expected to lead to a) local instrumentation methods for detailed interfacial characteristics, b) data for local interfacial area, void fraction, relative velocity and wave propagation velocity, and c) phenomenological models for interfacial area and interfacial structural changes.

\section{University Of Wisconsin}

Dept of Chemical Engineering

Madison, WI 53706

$\$ 108,337$

03-A

89-3

New Process Modeling, Design and Control

Strategles for Energy Efficiency, High

Product Quality, and Improved Productivity in the Process Industrles

W. Ray

The process industries are having great difficulty competing in the world market because of high energy costs, high labor rates, and old technology for many processes. This project is concerned with the development of process design and control strategies for improving energy efficiency, product quality, and productivity in the process industry. In particular, (1) the resilient design and control of chemical reactors, and (2) the operation of complex processing systems, will be investigated. Major emphasis in part (1) will be on two important classes of chemical reactors: polymerization processes and packed bed reactors. In part (2), the main focus will be on developing process identification and control procedures which allow the design of advanced control systems based on limited process information and which will work reliably when process parameters change in an unknown manner. Specific topics to be studied include new process identification procedures, nonlinear controller designs, adaptive cuntrol methods, and techniques for distributed parameter systems. Both fundamental and immediately applicable results are expected. The theoretical developments are being tested experimentally on pilot scale equipment in the laboratory. These experiments not only allow improvements in theoretical work, but also represent real life demonstrations of the effectiveness of the methods and of the feasibility of implementing them in an industrial environment. The new techniques developed in this project will be incorporated into computer-aided design packages and disseminated to industry. Therefore, it is expected that the work will have an impact on industrial practice. 


\section{Budget Number Index}

\section{Mechanical Sciences:}

01-A Solid Mechanics: macroscopic aspects of elastic and plastic deformations, and crack propagation

01-B Heat Transfer

01-C Fluid Mechanics

01-D Tribology

\section{Control Systems and Instrumentation:}

03-A Control systems, large scale systems

03-B Instrumentation for hostile environment, and NDE

03-C Intelligent systems

\section{Engineering Data and Analysis:}

06-A Thermophysical properties and processes

06-B Combustion

06-C Non-linear dynamics and engineering analysis 


\section{$01-\mathbf{A}$}

Chaos in Fluid-Structure Systems $\ldots \ldots \ldots \ldots \ldots \ldots \ldots \ldots \ldots$

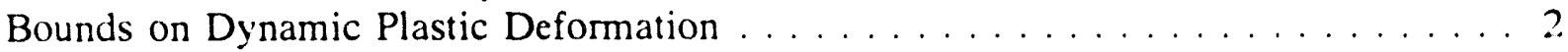

Continuum Damage Mechanics II . . . . . . . . . . . . . . . . . . 2

An Investigation of the Effects of History Dependent Damage in Time Dependent

Fracture Mechanics ............................ 3

Micromechanical Viscoplastic Stress-Strain Model with Grain Boundary Sliding . . 12

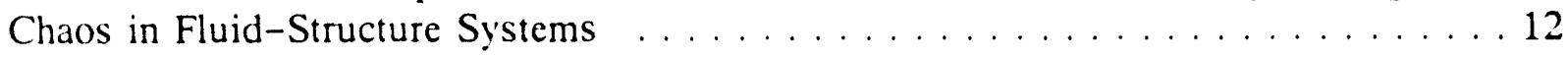

A Micromechanical Viscoplastic Stress-Strain Model with Grain Boundary Sliding . 14

Elastic-Plastic Fracture Analysis Emphasis on Surface Flaws . . . . . . . . . . . 17

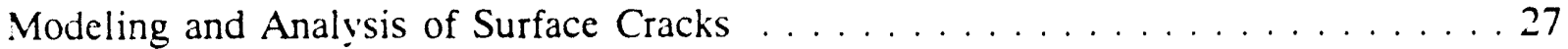

Basic Engineering Sciences of Solids Comminution $\ldots \ldots \ldots \ldots \ldots \ldots$

Inelastic Deformation and Damage at High Temperature . . . . . . . . . . . 40

Stochastic Quadrization for Probabilistic Analysis of Large Compliant

Off-Shore Structures . . . . . . . . . . . . . . . . . . . . 42

Stability and Stress Analyses of Surface Morphology of Elastic and

Piezoelectric Materiais . . . . . . . . . . . . . . . . . . . . . . 45

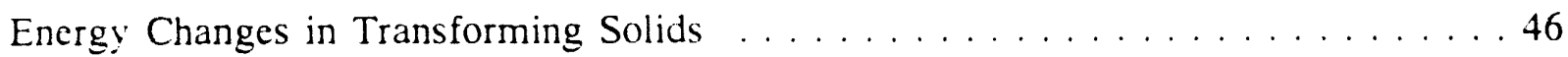

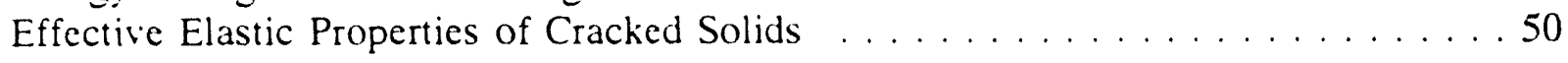

01-B

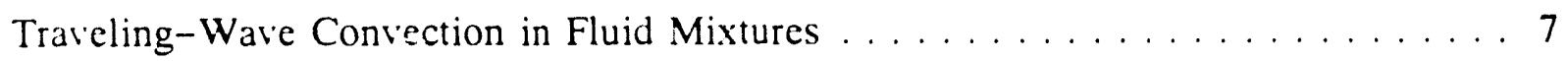

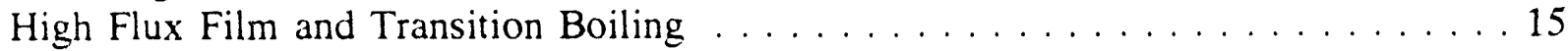

Momentum and Heat Transfer Processes in Viscoelastic Fluids . . . . . . . . . . . 19

The Impact of Separated Flow on Heat and Mass Transfer . . . . . . . . . . . . . . 29

Thinning And Rupture of Thin Liquid Films . . . . . . . . . . . . . . 34

Radiative Transfer Through an Array of Discrete Surfaces . . . . . . . . . . . 37

Radiative Transfer Through Arrays of Discrete Surfaces with Fixed Oricntation . . . . 37

Ultimate Limits of Boiling Heat Fluxes . . . . . . . . . . . . . . . . . 40

Heat Transfer in Three-Dimensional Turbulent Boundary Layers . . . . . . . . . 45

Fluid Dynamics of Double Diffusive Systems . . . . . . . . . . . . . . 47

Mass and Energy' Coupling Effects in Multiphase Free Shear Flows . . . . . . . 51 
Hydrodynamic Instabilities and Coherent Structures $\ldots \ldots \ldots \ldots \ldots \ldots$

Basic Studies of Transport Processes in Porous Media . . . . . . . . . . . . 5

Turbulence Structure and Transport Processes in Wary Liquid Strcams . . . . . . . 8

Lift and Drag Forces on Droplets and Particles in Wall-Bounded Flows . . . . . . 11

Experiments in Turbulent Mixing ..................... 13

Two Phase Potential Flow . . . . . . . . . . . . . . . . . . . . 14

Analytical and Experimental Study of Instabilities in Buoyancy-Driven Convection

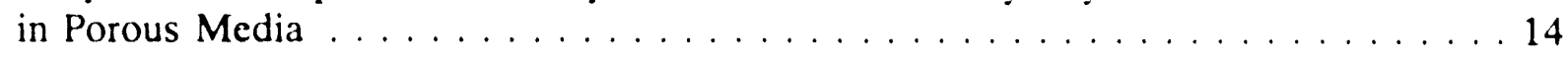

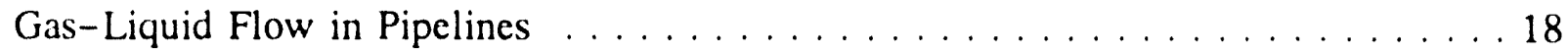

Numerical and Physical iviudeiing of Bubbly Flow Phenomena . . . . . . . . . . 20

Turbulence Theory and Reduced Hydrodynamic Description . . . . . . . . . . 20

Two-Phase Flow Measurements by NMR . . . . . . . . . . . . . . 21

Lagrangian Analysis of Contaminant Dispersal in Bounded Turbulent Shear Flows . . 23

Stability and Heat Transfer in Time-Modulated Flows . . . . . . . . . . . . . . 24

Dryout in Horizontal and Almost Horizontal Heated Tubes . . . . . . . . . . . 26

Rheological and Flow Characteristics of Dense Multiphase Slurries Employing a

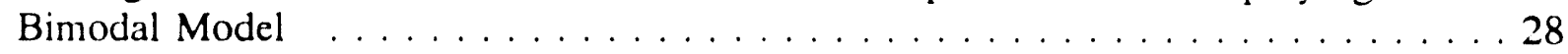

Stability Studies of Core-Annular Flows . . . . . . . . . . . . . . . . . . 29

Convection and Dispersion in Coarse-Packed Beds . . . . . . . . . . . 30

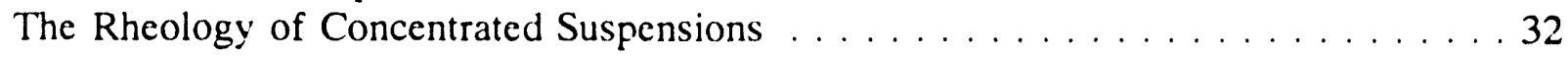

Effects of Capillarity of Macroscopic Flow in Porous Media . . . . . . . . . 34

Study of Interfacial Behavior in Cocurrent Gas-Liquid Flows . . . . . . . . . 35

Effect of Forced and Natural Convection on Solidification of Binary Mixtures . . . . 39

Interfacial Area and Interfacial Transfer in Two-Phase Flow . . . . . . . . . . . . 40

Microcomputer Enhanced Optical Investigation of Spreading and Evaporation

Processes in Ultra-Thin Films . . . . . . . . . . . . . . . . . . 41

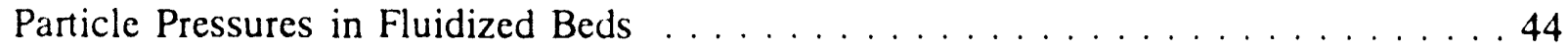

Numerical and Physical Modeling of Bubbly Flow Phenomena . . . . . . . . . . 49

Interfacial Area and Interfacial Transfer in Two-Phase Flow Systems . . . . . . 52

01-D

Cryotribology: Development of Cryotribological Theories and Application to

Cryogenic Devices

03-A

Modeling for Process Control . . . . . . . . . . . . . . . . . . . 9

Integration of Redesign Methodologies for Chemical Processes . . . . . . . . . . 9

Intelligent Control of Thermal Processes . . . . . . . . . . . . . . . . 17

Model Building, Control and Optimization of Large Scale Systems . . . . . . . 18

A General Procedure for the Synthesis of Process Flowsheets . . . . . . . . . . 24

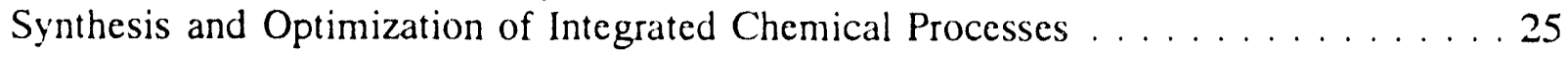

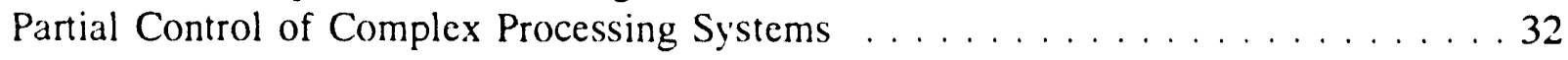


Monotonicity Analysis and Geometric Programming in Mechanical Shape

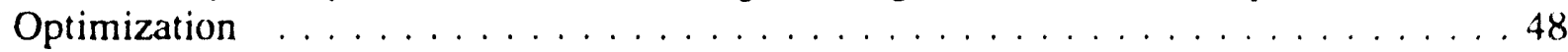

Laser Diagnostics Of PACVD Processes . . . . . . . . . . . . . . . . . .50

Dynamical Systems with Internal Structure: A New Approach to the Problem of

Analysis and Robust Design of a Time Varying System . . . . . . . . . . . . . 51

New Process Modeling, Design and Control Strategies for Energy Efficiency,

High Product Quality, and Improved Productivity in the Process Industries . . . . . 52

\section{3-B}

Nondestructive Evaluation of Superconductors $\ldots \ldots \ldots \ldots \ldots$

Pulse Propagation in Inhomogeneous Optical Fibers $\ldots \ldots \ldots \ldots \ldots \ldots$

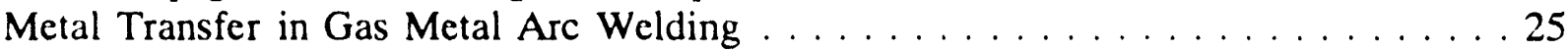

Multivariable Control Of The Gas-Metal Arc Welding Process . . . . . . . . . . 26

Development of Measurement Capabilities for the Thermophysical

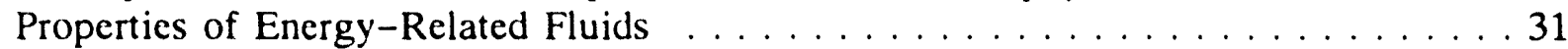

Quantitative Non-Destructive Evaluation of High Temperature

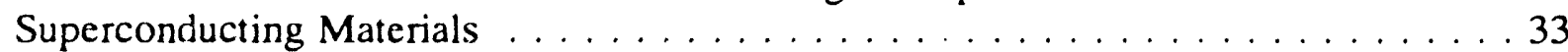

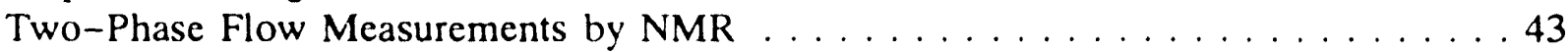

Application of Magnetomechanical Hysteresis Modeling to Magnetic

Techniques for Monitoring Neutron Embrittlement and Biaxial Stress . . . . . . . . 45

Nondestructive Testing . . . . . . . . . . . . . . . . . . . . . . . 46

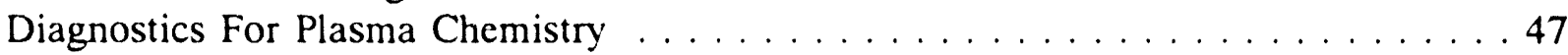

03-C

Research on a Reconfigurable Modular Manipulator System . . . . . . . . . . . . 10

Neural Leaming Formalisms for Global Manipulator Redundancy Resolution

Problems in Unstructured Environments . . . . . . . . . . . . . . . . . . . . . . . 19

The Use of Stereo Optical Flow Fields in the Determination of Three-Dimensional

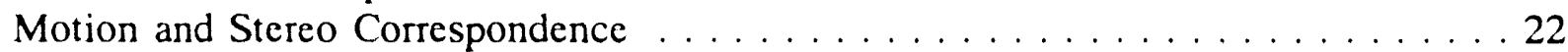

The Creation of Multi-Degree-of-Freedom Mechanisms for Robotic Applications . 33

Center for Engineering Systems Advanced Research (CESAR) . . . . . . . . . 35

\section{6-A}

Three Phase System Modelling .................... 15

In-Flight Measurement of the Temperature of Small, High Velocity Particles . . . . 16

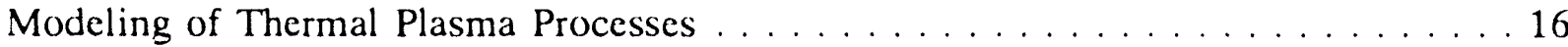

Transport Properties Of Disordered Porous Media From The Microstructure . . . . . 33

Bioprocess Engineering Research for Energy Applications . . . . . . . . . . . . 36

Experimental and Theoretical Studies of Condensation in Multicomponent Systems . . 38 
Experimental and Theoretical Study of Fuel Droplets Subject to Straining Flow . . . 6

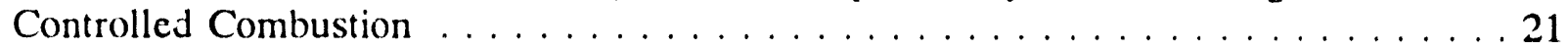

Nonuniform Liouville Transformers for Quasi-Homogeneous Optical Fields . . . . . 38

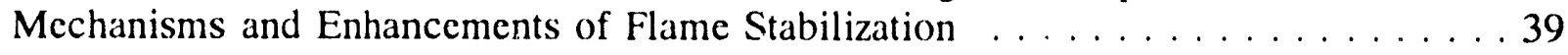

Mixing and Phase Change During Combustion . . . . . . . . . . . . . . 44

Structure of Inverse Diffusion Flames in Supercritical Fuel/Water Mixtures . . . . . 44

06-C

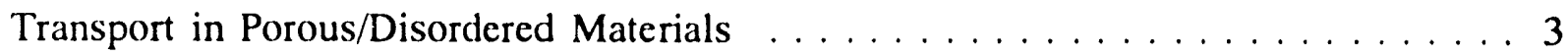

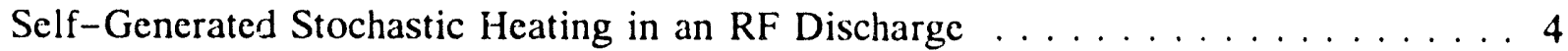

Energy and Particle Transport in Random Media ..... . . . . . . . . . . 5

Wave Turbulence and Self-Localization in Continuous Media . . . . . . . . . . 5

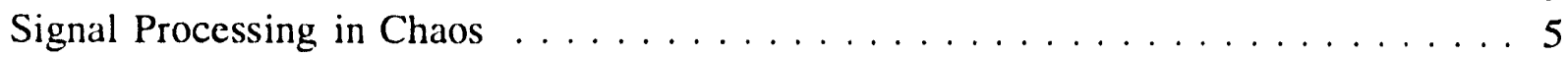

Noisy Nonlinear Systems . . . . . . . . . . . . . . . . . . . . 6

Knot Invariants and the Thermodynamics of Lattice Gas Automata . . . . . . . . . 6

Formation of Three-Dimensional Singularities, Regularization Procedures

and Markovian-Multifractal Description of Turbuient Flows . . . . . . . . 7

Bifurcations and Patterns in Nonlinear Dissipative Systems $\ldots \ldots \ldots \ldots$

Topics in Finite-Time Thermodynamics . . . . . . . . . . . . . . . 10

Fundamentals and Techniques of Nonimaging Optics for Solar Energy Concentration 11

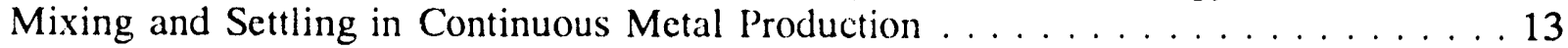

Studies in Nonlinear Dynamics . . . . . . . . . . . . . . . . . . 20

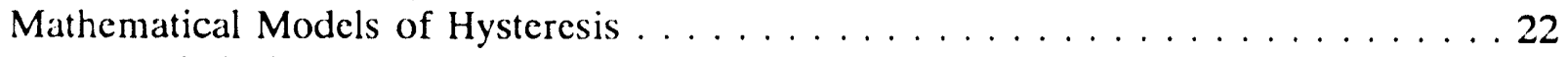

Macrostatistical Hydrodynamics . . . . . . . . . . . . . . . . . . . 29

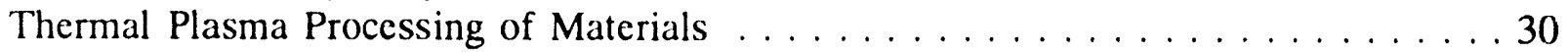

Low Resistivity Ohmic Contacts Between Semiconductors and High-Tc

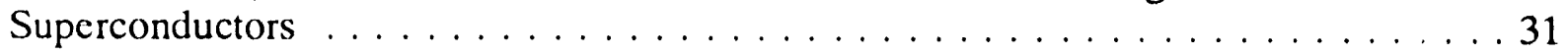

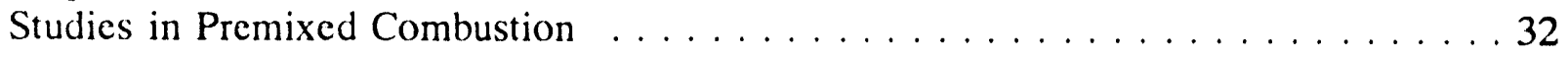

Mixing of Immiscible Fluids in Chaotic Flows and Related Issues . . . . . . . . 35

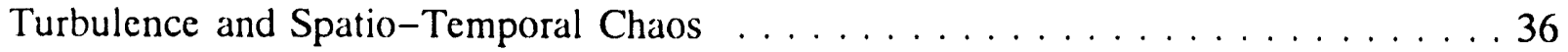

Radiative Interactions with Micromachined Surfaces:

Spectral Angular Polarized Emittance . . . . . . . . . . . . . . . . . 37

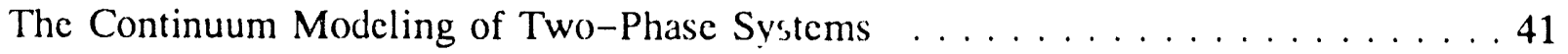

Flux Flow, Pinning, and Resistive Behavior in Superconducting Networks . . . . . 42

Coherence Effects in Radiative Energy Transfer . . . . . . . . . . . . . . . . . 42

Some Basic Research Problems Related to Encrgy . . . . . . . . . . . . . . 43

Thermodynamics in Combustion and Engines . . . . . . . . . . . . . 48

Investigation of Transitions From Order to Chaos in Dynamical Systems . . . . . . . 49

The Behavior of Matter Under Nonequilibrium Conditions: Fundamental Aspects

and Applications . . . . . . . . . . . . . . . . . . . . . . . . . 49

Complex Temporal and Spatial Patterns in Nonequilibrium Systems . . . . . . . . 50 


\section{Principal Investigators}

\begin{tabular}{|c|c|c|c|c|c|c|}
\hline H. Abarbanel & 5 & J. Georgiadis & 14 & C. Law & 39 & J. Ross \\
\hline J. A henbach & 33 & B. Ghosh & 51 & P. Libby & 6 & M. Sablik \\
\hline A. Acrivos & 32 & R. Givier & 43 & A. Lichtenberg & 4 & A. Sangani \\
\hline G. Ahlers & $\delta$ & R. Goldstein & 29 & M. Lieberman & 4 & S. Schladow \\
\hline S. Altobelli & 21 & A. Graham & 29 & K. Lindenberg & 6 & G. Schmidt \\
\hline F. Andrews & 15 & P. Triffith & 26 & R. Littlejohn & 20 & C. Scott \\
\hline J. Balint & 23 & I. Grossmann & 9 & W. Lloyd & 17 & J. Sengers \\
\hline S. Banerjee & 8 & T. Hanratty & 18 & M. Malone & 24 & S. Sheppard \\
\hline S. Bankoff & 34 & D. Hardt & 26 & R. Mann & 35 & R. Shinnar \\
\hline J. Barhen & 14 & J. Hartnett & 19 & I. Mayergoyz & 22 & G. Sivashinsky \\
\hline D. Barnett & 46 & S. Hay & 50 & F. McClintock & 27 & H. Smartt \\
\hline T. Basar & 18 & W. Haynes & 31 & M. McCready & 35 & P. Spanos \\
\hline A. Bergles & 40 & F. Hayot & 36 & J. McLaughlin & 11 & R. Steeper \\
\hline P Bernard & 23 & G. Herrmann & 46 & C. Menyuk & 22 & R. Street \\
\hline R. Berry & 10 & G. Hetsroni & 8 & D. Meyer & 6 & C. Surko \\
\hline L. Biegler & 9 & F. Incropera & 39 & M. Miksis & 34 & H. Swinney \\
\hline H. Brenner & 29 & M. Ishii & 40 & M. Moldover & 31 & S. Teitel \\
\hline F. Brust & 3 & Y. Iwasa & 27 & L. Mondy & 29 & K. Telschow \\
\hline C. Campbell & 44 & J. Jannson & 38 & F. Moon & 12 & C. Thompson \\
\hline D. Cannell & 8 & C. Jayaprakash & 36 & M. Morari & 9 & G. Thompson \\
\hline M. Cappelli & 47 & M. Jensen & 40 & J. Moreland & 31 & S. Torquato \\
\hline A. Caprihan & 21 & J. Johnson & 17 & T. Mulcahy & 1 & T. Troutt \\
\hline I. Catton & 4 & M. Jones & 30 & E. Novikov & 7 & L. Tsai \\
\hline C. Chang & 16 & E. Jordan & 12 & A. Oppenheim & 21 & K. Walker \\
\hline S. Chen & 1 & D. Joseph & 29 & F. Otter & 50 & J. Wallace \\
\hline E. Cohen & 43 & M. Kachanov & 50 & J. Ottino & 35 & G. Wallis \\
\hline S. Davis & 34 & T. Kanade & 10 & D. Parks & 27 & Z. Warhaft \\
\hline J. Douglas & 24 & A. Kaufman & 20 & C. Peterson & 28 & P. Wayner, Jr. \\
\hline D. Drew & 41 & R. Kayser & 31 & T. Petrosky & 49 & J. Welty \\
\hline M. Drost & 37 & A. Kerstein & 44 & E. Pfender & 30 & A. Westerberg \\
\hline J. Duncan & 22. & T. Keyes & 3 & F. Pin & 35 & D. Wilde \\
\hline T. Eagar & 25 & P. Khosla & 13 & G. Pomraning & 5 & G. Wilemski \\
\hline J. Eaton & 45 & G. Kino & 46 & I. Prigogine & 49 & F. Williams \\
\hline A. Eckbreth & 50 & G. Kojasoy & 52 & R. Probstein & 28 & R. Winston \\
\hline J. Ekin & 31 & P. Kokotovic & 18 & A. Prosperetti & 20 & L. Witte \\
\hline J. Epstein & 17 & J. Koseff & 47 & S. Putterman & 5 & E. Wolf \\
\hline L. Evans & 25 & R. Kraichnan & 20 & J. Ramshaw & 16 & J. Wolfe \\
\hline J. Fincke & 16 & D. Krajcinovic & 2 & W. Ray & 52 & C. Youngdahl \\
\hline A. Frenkel & 1 & E. Krempl & 40 & W. Reuter & 17 & J. Zemel \\
\hline M. Frish & 38 & C. Kruger & 47 & H. Richter & 13 & \\
\hline ․ Fukushima & 21 & R. Lahey, Jr. & 41 & I. Rinard & 32 & \\
\hline H. Gao & 45 & J. Lang & 25 & W. Roman & 50 & \\
\hline
\end{tabular}



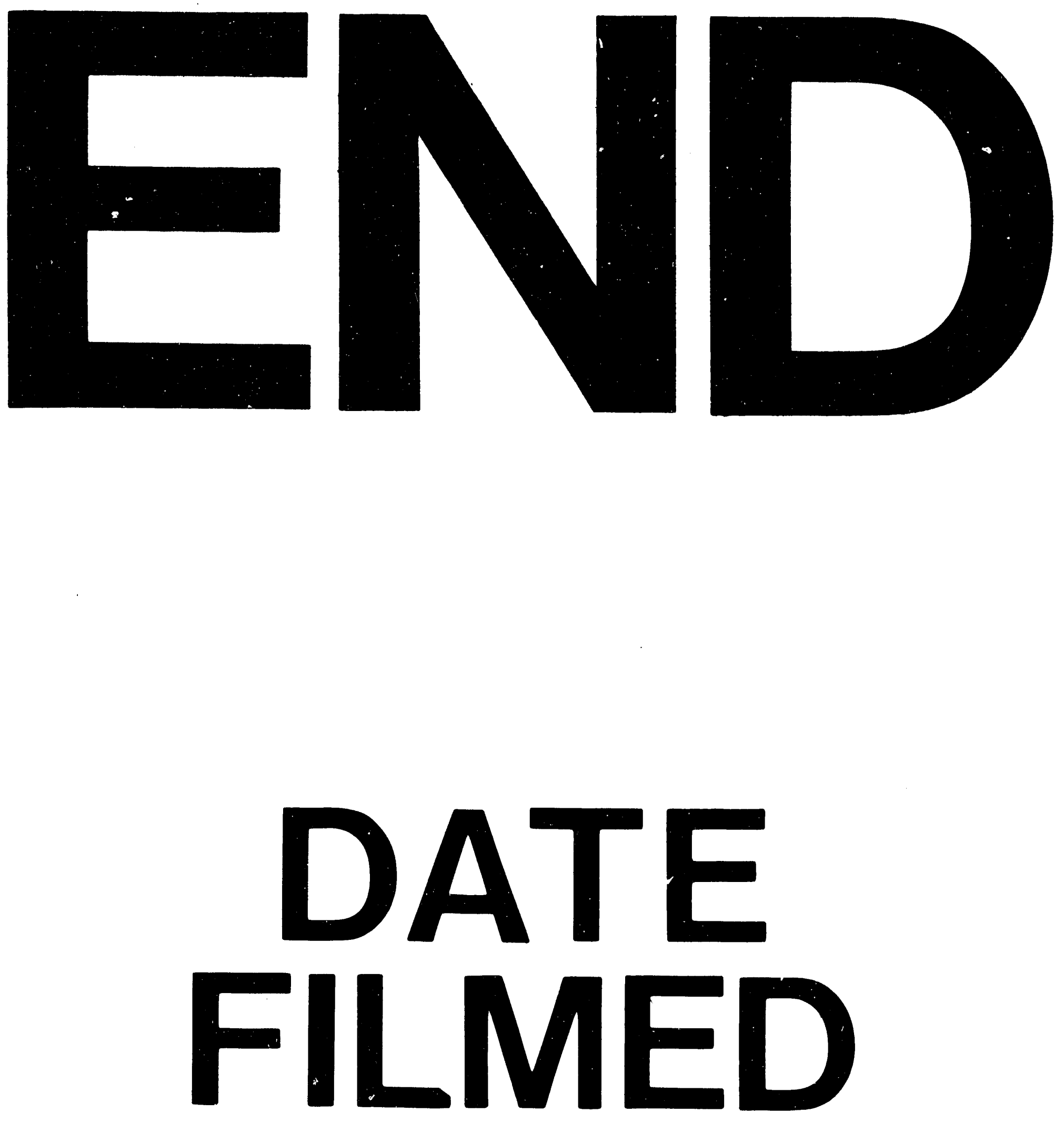

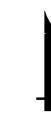

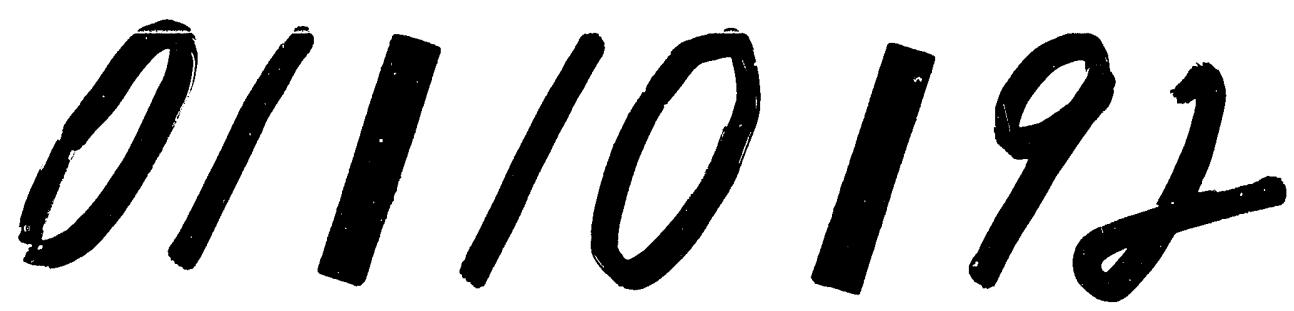


